\title{
The Assessment of In-Bed Mobility with Pressure Sensitive Mats
}

\author{
by
}

\author{
Stephanie Bennett, B.Eng.
}

A thesis submitted to the Faculty of Graduate Studies and Postdoctoral Affairs in partial fulfillment of the requirements for the degree of

\section{Masters of Applied Science in Biomedical Engineering}

Ottawa-Carleton Institute for Biomedical Engineering

Department of Systems and Computer Engineering

Carleton University

Ottawa, Ontario, Canada, K1S5B6

December 2013

(C) Copyright 2013 Stephanie Bennett 


\section{Abstract}

Clinical mobility tools have been shown to predict adverse outcomes in elderly patients, yet aren't used often enough to inform hospital staff on patient health. Integrated computing has therefore become increasingly important and is predicted to improve traditional healthcare. This thesis details the design of an algorithmic system to partially automate a mobility tool.

Three pressure sensitive mats were set-up on a hospital bed frame, underneath a mattress. Thirty volunteers enacted five movements on the hospital bed; each movement representative of a different mobility score. These movements generated pressure data, and a system of algorithms was constructed in a decision tree to automatically classify data. The overall system yielded $96 \%$ accuracy, where the misclassifications were due largely to inconsistencies in volunteer performance. These results suggest that this algorithmic system is effective in distinguishing between the mobility enactments examined here, and emphasizes the potential for integrated computing to improve traditional healthcare. 


\section{Acknowledgements}

First and foremost I would like to thank my academic supervisor, Dr. Rafik Goubran for the enormous support I received throughout my thesis. I encountered a number of challenges in the last year and Dr. Goubran's unfailing support and patience were immeasurable in the completion of my thesis. I would also like to thank my medical supervisors, Dr. Kenneth Rockwood and Dr. Frank Knoefel, for their professional mentoring, for always having confidence in my abilities, and for always taking time from busy schedules to offer their expertise on matters relating to my thesis.

Thanks are also due to Amaya Arcelus for helping this project take flight, Bruce Wallace for answering many of my endless questions, and Megan Holtzmann for answering all my other endless questions. I would also like to thank Dr. Arnold Mitnitski, Sherri Fay, Jody Wells, Mike Rockwood, Lindsay Wallace and all the other members of the Geriatric Medicine Research Unit in Halifax, NS, for their time, friendship, and for helping me through hospital ethics applications, ethics amendment applications and volunteer data collection.

Finally, I would like to thank my family and dear friends for giving me so much unconditional love, support and encouragement during one of my hardest years. I honestly would not have been able to complete this thesis without them. 


\section{Table of Contents}

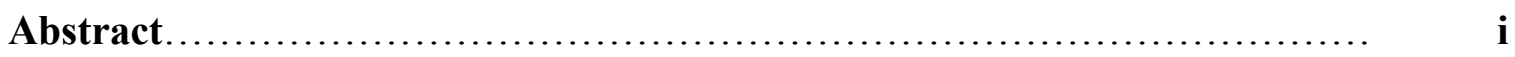

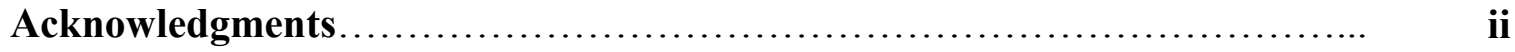

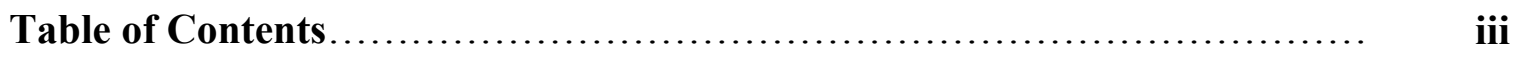

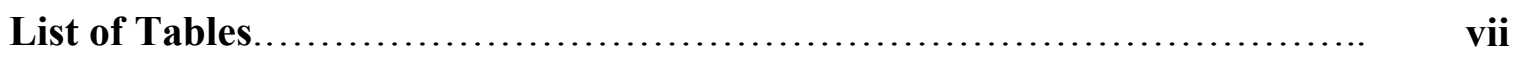

List of Illustrations.................................................. viii

List of Appendices................................................

List of Acronyms..................................................

Chapter 1: Introduction............................................ 1

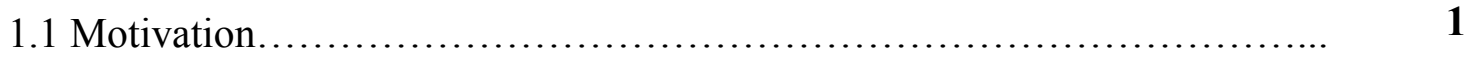

1.2 Problem Statement................................................

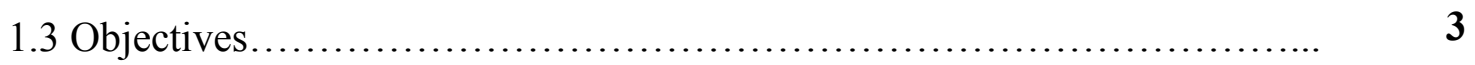

1.4 Summary of Contributions....................................... 4

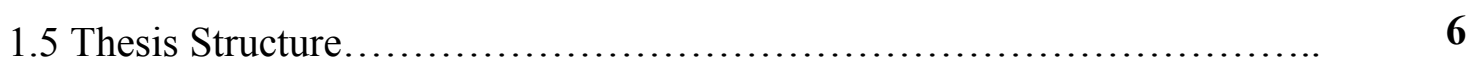

Chapter 2: Background ............................................. 7

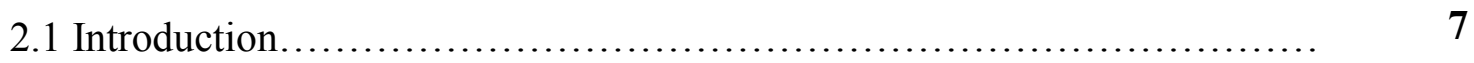

2.2 Impaired Mobility and Frailty.................................... $\quad 7$

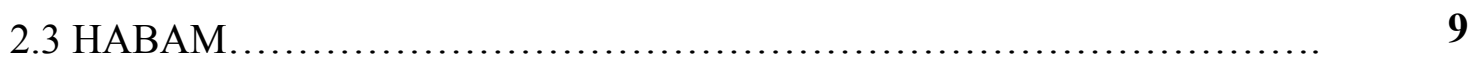

2.4 Patient Monitoring and Pressure Sensing.............................. 11

2.5 Fiber Optic Sensors............................................... 12 
2.6 Related Work

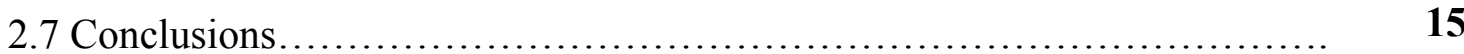

Chapter 3: Equipment and Experimentation............................ 16

3.1 Introduction..................................................... $\quad 16$

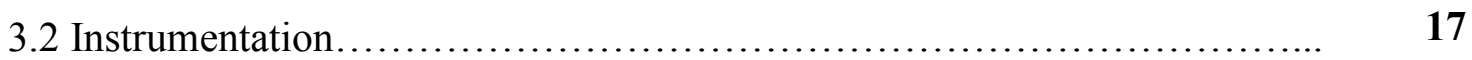

3.3 Equipment Set-Up............................................ 20

3.4 Experimentation.................................................. 21

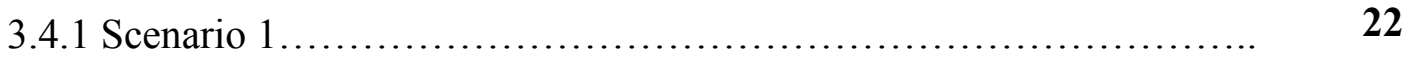

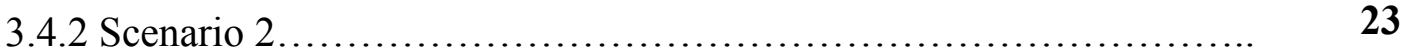

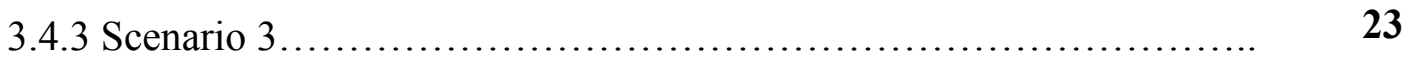

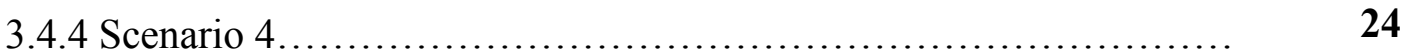

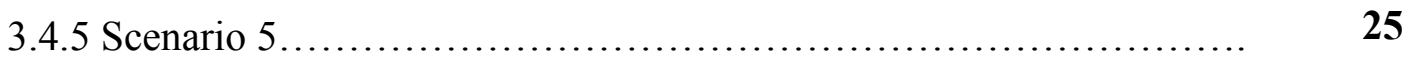

3.5 Data Organization and Calibration................................... 26

3.5.1 Organization of Data......................................... $\quad 26$

3.5.2 Data Calibration............................................. 31

Chapter 4: Algorithmic System Set-Up............................ 33

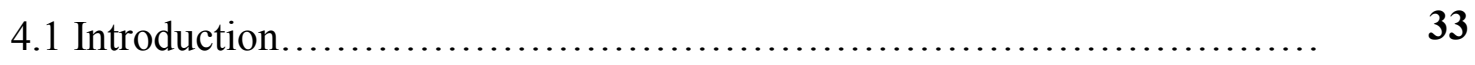

4.2 System Set-Up............................................... 33

Chapter 5: Determination of Initial Posture............................ 36

5.1 Introduction.................................................. 36

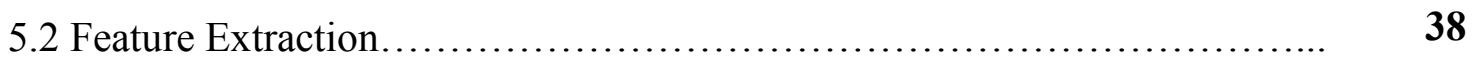

5.2.1 PAS: Percentage of Active Sensors............................. 39 
5.2.2 LonCP: Longitudinal Center of Pressure.

5.2.3 LatCP: Latitudinal Center of Pressure.

5.2.4 LonV: Longitudinal Variance.

5.2.5 LatV: Latitudinal Variance

5.2.6 CLR: Central Left Ratio

5.2.7 QLR: Quarter Left Ratio....

5.3 Feature Evaluation and Selection.

5.4 Results and Discussion

Chapter 6: Distinction Between Lying Scenarios........................ 56

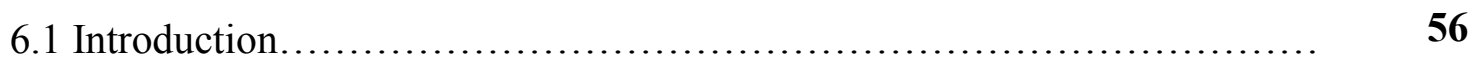

6.2 Head Mat Pressure Monitoring..................................... 58

6.3 Region Based Pressure Monitoring.................................. 61

6.4 Algorithmic Results and Discussion............................... $\quad 66$

Chapter 7: Distinction Between Sitting Scenarios....................... 70

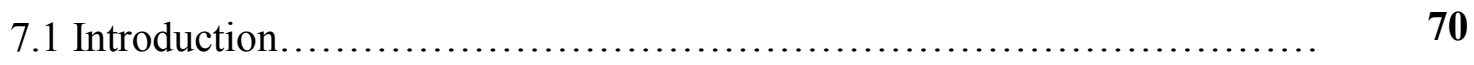

7.2 Feature Extraction............................................... $\quad 71$

7.2.1 SVL: Sum of Vector Lengths............................... 73

7.2.2 NAA: Number of Acute Angles.............................. 74

7.2.3 SFV: Start to Finish Vector.................................. $\quad 75$

7.2.4 LSV: Longest Single Vector............................... $\quad 75$

7.2.5 PA: Polygon Area............................................ $\quad 76$

7.2.6 NSC: Number of Spatial Coordinates.......................... $\quad 76$

7.3 Feature Evaluation and Selection................................... 77 


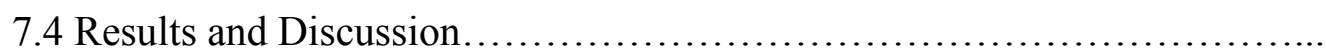

Chapter 8: System Performance and Conclusions.......................... 81

8.1 System Performance.............................................. 81

8.2 Thesis Conclusions............................................. 82

8.3 Thesis Contributions............................................ 85

8.4 Future Research.................................................. 86

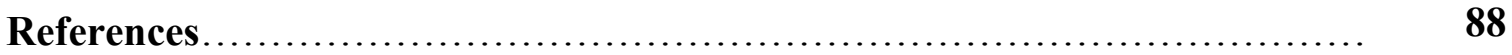

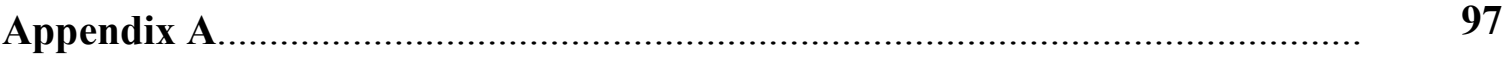




\section{List of Tables}

Table 1: Key terms and the designated notations …………...................................... 26

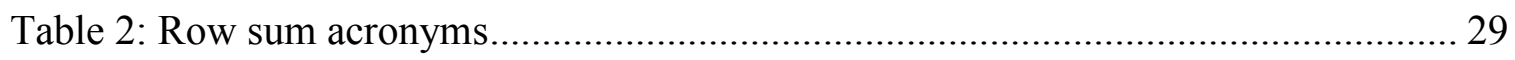

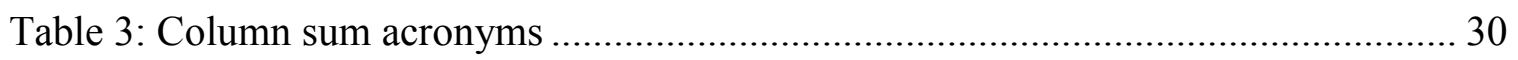

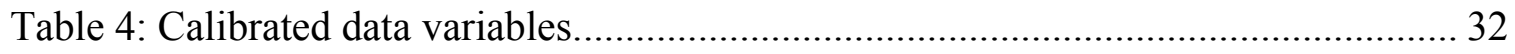

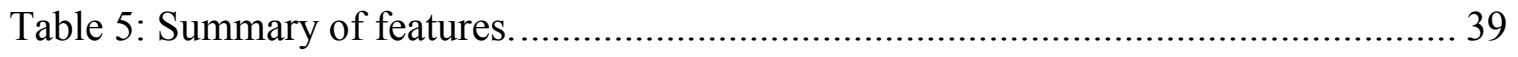

Table 6: Features examined and the corresponding classifier accuracies......................... 52

Table 7: Features and resulting accuracies after the addition of noise. ........................... 53

Table 8: Summary of variable acronyms for DBLS ……………………………….... 57

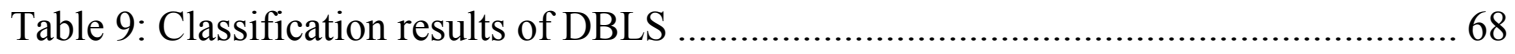

Table 10: Summary of features examined for DBSS................................................... 73

Table 11: Features examined and respective classifier accuracies................................. 78

Table 12: Accuracies of features evaluated by both classifiers. ....................................... 78

Table 13: Accuracies of combined features evaluated by both classifiers. ...................... 78

Table 14: True classifications verses system classifications. ......................................... 82 


\section{List of Illustrations}

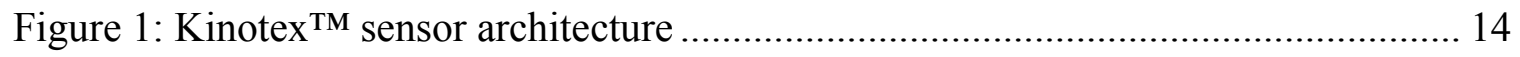

Figure 2: A reference schematic of the hospital bed in the experimentation room.......... 17

Figure 3: A single pressure mat and the dimensions. .................................................. 18

Figure 4: Spacing of the sensors within the pressure mat............................................. 18

Figure 5: Schematic of a single pressure sensor embedded in polymer foam................. 19

Figure 6: The bed fame dimensions and the placement of pressure mats on the frame... 20

Figure 7: Schematic of Scenario 1 movements........................................................... 22

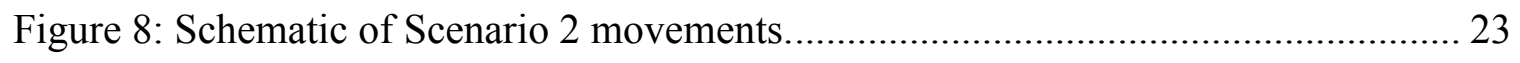

Figure 9: Schematic of Scenario 3 movements.......................................................... 24

Figure 10: Schematic of Scenario 4 movements.......................................................... 25

Figure 11: Consideration of mats as one data array.................................................. 27

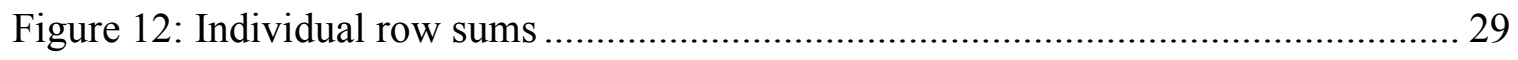

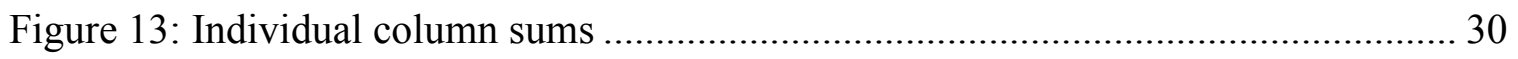

Figure 14: System tree describing the decision process for the entire algorithmic system.

Figure 15: System tree describing the decision process for DIP as it applies to the whole

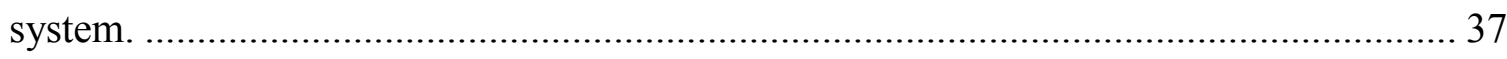

Figure 16: A subject in a supine lying position. …………........................................... 37

Figure 17: A subject in a sitting position on the left edge of the bed. .............................. 37 
Figure 18: Average still data from Participant 5 lying supine on the bed with a zero score threshold. 41

Figure 19: NAS at varying threshold values for grouped tests 43

Figure 20: Group average NAS and NAS differences between the two motion groups at varying threshold values.

Figure 21: NAS for Participant 5 in a supine lying posture with a zero and individualized threshold. 45

Figure 22: Schematic of pressure mats as they are placed on the bed frame. 46

Figure 23: Schematic of the pressure mats with a highlighted central left area. 49

Figure 24: Schematic of the pressure mats with a highlighted quarter left area. 50

Figure 25: All data from the features with total accuracy 53

Figure 26: Final classified data and classifier threshold 54

Figure 27: System tree describing the role of DBLS respective to the entire system. .... 56

Figure 28: System tree describing the architecture of Subsystem 2. 57

Figure 29: Schematic of the mats for head mat pressure monitoring. 59

Figure 30: Schematic of the groupings for region based pressure monitoring. 62

Figure 31: Schematic of the significant verses insignificant sensor scores. 64

Figure 32: Significant (Inside) verses insignificant (Outside) sensor scores as subjected to a classifier 65

Figure 33: Percentage pressure change from baseline for the top mat. 66

Figure 34: Two incidences of five simultaneous pressure sensors experiencing pressure relief. 67

Figure 35: Schematic of the five sensors shown to have been relieved. 68 
Figure 36: System tree describing the decision process for DBSS as it applies to the

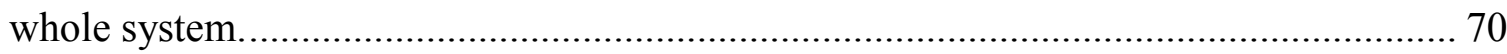

Figure 37: Mapped center of pressure for the duration of scenarios 4 and 5.................. 72

Figure 38: Zoomed, mapped center of pressure...................................................... 72

Figure 39: Final SVM model and resulting classifications....................................... 79 


\section{List of Appendices}

Appendix A: HABAM form 


\section{List of Acronyms}

\begin{tabular}{|c|c|}
\hline R1 & Sum Row 1 \\
\hline $\mathbf{R 2}$ & Sum Row 2 \\
\hline $\mathbf{R 3}$ & Sum Row 3 \\
\hline R4 & Sum Row 4 \\
\hline R5 & Sum Row 5 \\
\hline R6 & Sum Row 6 \\
\hline R7 & Sum Row 7 \\
\hline R8 & Sum Row 8 \\
\hline R9 & Sum Row 9 \\
\hline C1 & Sum Column 1 \\
\hline $\mathrm{C} 2$ & Sum Column 2 \\
\hline $\mathbf{C 3}$ & Sum Column 3 \\
\hline $\mathrm{C} 4$ & Sum Column 4 \\
\hline C5 & Sum Column 5 \\
\hline C6 & Sum Column 6 \\
\hline C7 & Sum Column 7 \\
\hline C8 & Sum Column 8 \\
\hline CISD & Calibrated Individual Sensor Data \\
\hline r1, r2, etc. & Calibrated Row Sum \\
\hline c1, c2, etc. & Calibrated Column Sum \\
\hline DIP & Determination of Initial Pressure \\
\hline DBLS & Distinction Between Lying Scenarios \\
\hline DBSS & Distinction Between Sitting Scenarios \\
\hline PAS & Percentage of Active Sensors \\
\hline LonCP & Longitudinal Center of Pressure \\
\hline LatCP & Latitudinal Center of Pressure \\
\hline
\end{tabular}




\begin{aligned} & LonV Longitudinal Variance \\ & LatV Latitudinal Variance \\ & CLR Central Left Ratio \\ & QLR Quarter Left Ratio \\ & SMA Sum of Sensors in Mat A \\ & BMA Baseline of Mat A \\ & PIMA Percentage Increase of Mat A \\ & PDMA Percentage Decrease of Mat A \\ & BPI Baseline Pressure for Individual sensors \\ & PII Pressure Increase for Individual sensors \\ & PDI Pressure Decrease for Individual sensors \\ & SVL Sum of Vector Lengths \\ & NAA Number of Acute Angles \\ & SFV Start to Finish Vector \\ & LSV Longest Single Vector \\ & PA Polygon Area \\ & NSC Number of Spatial Coordinates \\ & \hline\end{aligned}




\section{Chapter 1}

\section{Introduction}

This chapter details the motivation for this thesis, reviews the problem statement and objectives as well as states contributions and describes the thesis structure.

\subsection{Motivation}

Statistics Canada reported in 2010 that the predicted number of seniors will surpass the number of children aged 14 and under for the first time in history, sometime between 2015 and 2021 [1]. One year later, in 2011, Statistics Canada reported that the rate of growth in seniors aged 65 and older (14.1\%) had surpassed that of children aged 14 and under $(0.5 \%)$ and people aged 15 to $64(5.7 \%)$ [2]. Such statistics illustrate Canada's rapidly aging population. This demographic shift is expected to have a profound impact on individuals, communities and health services, shaping our society over the next 20 to 30 years [3]. The impending demographic shift is a well recognized phenomenon; nationwide strategies have been developed to help Canada prepare for the challenges it presents [3],[4]. An integral part of these strategies are a strong support for biomedical engineering research in the field of geriatrics. One of the major issues of an aging population is an increased patient to healthcare provider ratio. This could mean reduced access to healthcare providers and the possibility of compromised patient healthcare. Biomedical engineers are working to uphold the quality of healthcare by integrating 
automated patient monitoring and assessments, which allows for better allocation of time and resources within a hospital. This work looks to not only uphold, but improve the quality of healthcare by harnessing the innate ability of technology to capture what human senses cannot.

\subsection{Problem Statement}

Functional impairment and impaired mobility have been considered geriatric 'giants' since before 1997 [5],[6]. In older patients, impaired mobility is a complex phenomenon and can often be a sign of underlying disease [7]. Methods of assessing a patient's ability to move have therefore been of considerable interest to health professionals in determining older patients' health. The Hierarchical Assessment of Balance and Mobility (HABAM) is an assessment tool developed to assess, track, and therefore manage patient health by observing patient ability, then allocating a score once per day from hospital admission to discharge [8]. Progressions or declines in patient health are numerically and graphically represented. The HABAM tool is set apart from other mobility assessment tools, as it allows for the assessment of bedridden patients as well as patients who are only mildly functionally impaired [9].

While the HABAM tool has been shown to be reliable, responsive, sensible, as well as a predictor of adverse outcomes [10],[11], it is not currently used enough by hospital staff to be informative. This is not an uncommon problem in modern hospitals; proportionally, populations are aging at a rapid pace, overloading health resources and staff [12]. The addition of any clinical tool that takes nurses away from patient care is not currently a popular idea. Many hospitals are therefore integrating automated computing 
into hospital and home environments to help care for increasing numbers of older patients while maintaining the quality of healthcare. The goal of this work is often not only to automate, but to improve current health measurements. Particular to impaired mobility and immobility, much work has been completed with accelerometers or pressure sensors. This is inclusive of: monitoring and detecting sleep patterns [13],[14], monitoring and detecting gait patterns [15],[16], pressure ulcer prevention [17],[18] and 'smart home' environments [19],[20], among other research.

Considering the implications of immobility, the lack of clinical tools developed to monitor it, and the successful use of integrated computing, the automation and integration of HABAM may be an important informative addition to many hospitals.

\subsection{Objectives}

The main objective of this work is to automatically quantify the mobility of a patient in bed. The algorithmic system developed to achieve this uses the HABAM tool to quantify mobility, and pressure sensor signals to measure movement. Volunteers performed in-bed motions representative of patients of varying movement abilities (corresponding to in-bed HABAM scores). For example, a patient that is able to independently sit up in bed would receive a HABM Mobility score of 7, whereas a patient who couldn't move at all would receive a HABAM Mobility score of 0 . Pressure mats were set-up underneath the hospital mattress to gather data during volunteer enactments. A system of algorithms was developed to take in movement data and output the correct HABAM score. 
The secondary objective of this work is to gain insight on small measures of mobility and explore the possibility of expansion of the HABAM to include more graded measurements of mobility.

\subsection{Summary of Contributions}

This thesis was built upon several stages of research. The contributions presented in this section are representative of these stages and include:

1. The first contributions were preliminary work and data collection. It was demonstrated in a paper on frailty in elderly patients that a limit to frailty as measured by the Frailty Index [8], exists in Chinese adults aged 80 and over, having important implications as to how health should be understood in this age bracket. Pressure data collection was from 30 volunteers, performing 5 scenarios each for a total of 150 individual sets of data. There were an additional 150 sets of corresponding video footage.

- [21] S. Bennett, X. Song, A. Mitnitski, K. Rockwood, "A Limit to Frailty in Very Old, Community-Dwelling People: A Secondary Analysis of the Chinese Longitudinal Health and Longevity Study," Age and Ageing, pp. 1-6, December 2012.

2. The second contribution was the primary stage of the overall system, called the Determination of Initial Posture. This stage used feature extraction and pattern classification to determine between one of two initial volunteer postures; a supine lying position and a sitting position.

- [22] S. Bennett, R. Goubran, K. Rockwood, A. Arcelus, and F Knoefel, "Pressure Signal Feature Extraction for the Differentiation of Clinical 
Mobility Assessments," in Proc. IEEE Int. Symp. on Medical Measurements and Applicat. (MeMeA), Budapest, Hungary, 2012, pp. 1-5.

- [23] S. Bennett, R. Goubran, K. Rockwood, and F Knoefel, "Automated Assessment of Mobility in Bedridden Patients," in Proc. of the $35^{\text {th }}$ Annu. Int. Conf. of IEEE Eng. in Medicine and Biology Soc. (EMBC), Osaka, Japan, 2012, pp. 4271-4274.

3. The third contribution was one of two secondary stages of the overall system, called the Distinction Between Lying Positions. This stage used regions of interest and ratio calculations to identify incidences of pressure point relief and determine which of three scenarios (with an initial lying position) a given set of data represented.

- [24] S. Bennett, R. Goubran, K. Rockwood, and F Knoefel, "Monitoring the Relief of Pressure Points for Pressure Ulcer Prevention: A Subject Dependant Approach," in Proc. IEEE Int. Symp. on Medical Measurements and Applicat. (MeMeA), Gatineau, QC, 2013, pp. 135-138.

- [25] S. Bennett, R. Goubran, K. Rockwood, and F Knoefel, "Considering Center of Gravity in Pressure Feature Extraction From Mobility Assessments," presented at the 2nd annual conference of Healthcare Support through Inform. Technology Enhancements (hSITE), Montreal, QC, 2012.

4. The fourth contribution was the second of the two secondary stages, called the Distinction Between Sitting Scenarios. This stage calculated the center of pressure underneath subject, then used feature extraction and pattern classification to 
determine which of two scenarios (with an initial sitting position) a given set of data represented.

- [26] S. Bennett, R. Goubran, K. Rockwood, and F Knoefel, "Distinguishing Between Stable and Unstable Sit-To-Stand Transfers Using Pressure Sensors," submitted to IEEE Int. Symp. on Medical Measurements and Applicat. (MeMeA).

\subsection{Thesis Structure}

This thesis is organized into eight total chapters. A detailed background on frailty in older adults, the HABAM tool and pressure sensing is given in Chapter 2. Chapter 2 is followed by a description of the equipment set-up and experimental procedures used to generate and gather data in Chapter 3. Chapter 4 describes the set-up of the algorithmic system, which is broken into two stages. The first stage of the system is described in Chapter 5, followed by subsequent stages of the system in Chapters 6 and 7. In particular Chapter 5 uses feature extraction and classification to classify an unknown data set into one of two groups. Based on this classification data is then passed to the second stage, which consists of two parts detailed in Chapter 6 and 7. Chapter 6 uses region based pressure monitoring to identify which of three movements (the first grouping) unknown data represents. Chapter 7 calculates center of pressure, then uses feature extraction and classification to identify which of two movements unknown data represents (the second grouping). 


\section{Chapter 2}

\section{Background}

This chapter details background information needed to fully understand the motives behind this thesis project and the previous work upon which this project is built.

\subsection{Introduction}

An overview of the background information surrounding this thesis topic is discussed in the following sections. Sections 2.2 and 2.3 discuss atypical disease presentation in older adults, how frailty and illness are quantified, and the potential for integrated computing to improve current measures. Sections 2.4 and 2.5 discuss the role of pressure sensing in hospital environments, fiber optic sensors, and the potential for these sensors to be used in the automation of HABAM. Finally, Section 2.6 discusses work completed with fiber optic pressure sensors in the field of geriatrics.

\subsection{Impaired Mobility and Frailty}

Disease presentation in elderly adults, especially those who are frail, is often unlike that of a younger population [10]. Where a young patient with pneumonia presents with classic clinical symptoms of fever, cough, increased mucus production, shortness of breath and inflammation of the lungs, frail older adults often have diminished or absent symptoms [27]. Typical 'atypical' disease presentations are known as the "geriatric 
giants" and are inclusive of: delirium, functional decline, social withdrawal, acute incontinence, and impaired mobility and balance [6]. The latter in particular is commonly seen as an inevitable part of aging, often obscuring its status as a sign of acute illness [28]. Given that about $20 \%$ of very old individuals ( $88+$ years) walk normally [29], impairments in mobility are not only a consequence of old age. Furthermore, elderly individuals with gait disorder have been found to have an increased risk of developing dementia and have reduced survival rates compared to their un-impaired peers, supporting the theory that declines in mobility are indeed signs of underlying disease [28],[30].

Impaired mobility is linked to frailty. At Geriatric Medicine Research (GMR) at Capital District Health Authority / Dalhousie University, frailty is described as the differential susceptibility of older adults of the same chronological age to adverse health outcomes [31]. This definition is well received in the scientific community [32]-[37] and is the basis for GMR's work on the frailty index, which has been independently validated by a dozen groups since 2005 [33],[36],[37]. While most diagnostic frailty tools focus on characteristic features common amongst sick, elderly individuals, the frailty index considers the number of things that are wrong with elderly individuals, or the number of deficits [31]. The greater the deficit count, the greater the risk of an adverse outcome such as institutionalization or death and the greater the state of frailty [8].

The frailty index has been proposed as a "clinical state variable". In physics and systems engineering a "state variable" refers to a variable that uniquely defines the system response at any given time [38]. Work done in GMR has attempted to expand the idea to include the clinical states of patients and hence the term "clinical state variable". 
Balance and mobility in elderly patients are high level functions that depend on a complex interaction between movement, cognition and mood [28], are indicators of illness and can therefore be considered as "clinical state variables". As such, the Hierarchical Assessment of Balance and Mobility (HABAM) was developed and is detailed in Section 2.3.

\subsection{HABAM}

The HABAM (Appendix A) is a clinical tool used to monitor patient health through the assessment of "clinical state variables". Patients are allocated HABAM scores every day over the duration of their hospital stay based on their abilities in static balance, dynamic balance and mobility, where higher scores indicate greater ability. The tool tracks changes in scores both numerically and graphically, thus providing an intuitive description of patients' abilities over time in three areas: mobility, transfers and balance [10]. The HABAM tool has been demonstrated to be valid, reliable, responsive, and sensible as a measure of mobility and balance [9],[39],[40].

Other tools have been developed to assess an elderly patients' health state by evaluating their physical abilities for signs of decline. These assessment tools include the Timed Up and Go test (TUG) [41], the Tinetti Balance and Mobility test [42], the Berg Balance scale (BBS) [43] and the Functional Reach test [44], among others. While these tests have been validated in older people, they cannot assess patients who are unable to stand or walk [10]. This is an important aspect of an elderly mobility assessment tool as those who are bed-fast are among the frailest, are at a higher risk for adverse outcomes and, therefore, need close monitoring for declines [9]. The HABAM tool allows for 
assessment of bedridden patients as it is sensitive to states in which a patient can or cannot relieve pressure points in bed while lying flat.

Tracking mobility over the course of a hospital stay is crucial in determining whether a patient's state of health is improving or declining, and at what rate change is occurring [8]. Although health pattern recognition is crucial for making clinical decisions, organization of information to facilitate pattern recognition is not well developed [8]. HABAM is designed to facilitate health pattern recognition as it both assesses and tracks progression and recovery in ill older adults. Recent findings show that decline in bed mobility within the first 48 hours of hospital admission is associated with a greatly elevated risk of 30-day mortality [11].

The HABAM tool is used in general medical and rehabilitation wards currently to further investigate patterns in HABAM scores, particularly in relation to patient frailty, and determine if the tools can provide insight into the value of different interventions for patients of differing frailty levels. The tool is implemented by medical staff, who observe and test patients then record scores by hand. In this way, this tool, and many other clinical tools, is limited to what can be observed by the human eye. Pervasive and ubiquitous computing is able to gather data unobservable to the human eye, offers continuous monitoring of chronic patients, can be used in a number of settings, and is thought to improve traditional health care due to analytical, diagnostic, supportive, information and documentary functions [45],[46]. Implications of such functions include disease management, early diagnosis and treatment, and therefore patient disease prevention as well as reduced expenses due to mismanaged diseases and reduced mental and physical overload of health professionals [46]. There has therefore been a recent 
surge of interest in pervasive/integrated computing, which has resulted in super sensitive technologies being used in hospital settings [45]. The following sections detail current research in pervasive computing, particularly in the area of pressure sensing.

\subsection{Patient Monitoring and Pressure Sensing}

Systems monitoring patient movement generally consist of a sensing and monitoring portion, using biomedical sensors or smart textile measurement devices, in conjunction with computing via laptops, microprocessors, or other methods [47]. Different types of sensing have been examined; accelerometry and pressure sensing are among the most widely examined and are emerging fields [48]. Accelerometry is reported to have the potential to be a practical approach to study activity levels of older patients in rehabilitation settings [48]. However, few available studies had recruited frail older patients as subjects, and the few that did had either a very small sample size or used multiple accelerometer devices [48]. Furthermore, measures of activity were inclusive of only general movements such as sitting-to-standing or lying-to-sitting, and accelerometry as a measure of balance and mobility requires that a patient wear an accelerometer device. Accelerometry is therefore undesirable for applications such as the HABAM tool; a finer grade of information is required to identify subtle differences in in-bed movements that differentiate scores, and placing a device on a frail patient in critical condition is undesirable. Pressure sensors on the other hand, do not come in contact with the patient at all and have a very fine sensitivity, enabling detection of patient rollovers at night, detection of movement onset times (movement initiation time), and even collection of respiratory rate information [49],[50],[51]. 
A number of pressure sensing methods are available and have been used in healthcare settings. Of particular importance to biomedical applications are methods using optical fibers [52]. Silica fibers in biomedical applications can be thinner than a standard surgical suture, can be used in a clinical setting without any electrical or chemical interference with existing technical set-ups, are intrinsically safe and can even be used for continuous in vivo monitoring [52],[53]. This technology has been implemented successfully for health monitoring in civil and aerospace applications, and recently has been investigated for applications in the field of medicine [53]. Emerging trends with optical fibers in the medical field include smart textiles, balance and gait analysis, smart beds and intelligent human machine interfaces [52]. This emerging work has resulted in smart wearable textiles that gather cardiac, respiratory and pulse information; gait analysis mats to better monitor patients with cerebral palsy, Parkinson's disease and neuromuscular disorders, and; the use of Fiber Bragg Grating (FBG) sensors to detect pressure ulcers, to improve amputee sockets and wheelchair seating systems [52]. Section 2.5 describes fiber optic sensors in more detail.

\subsection{Fiber Optic Sensors}

The basic working principle of fiber optic sensors is based on the modulation of an optical property of fiber-guided light [54]. For biomedical applications, most fiber optic sensors are of the light intensity modulation type due to simplicity and low cost. Several different fiber optic force/displacement sensors have been developed successfully for biomedical tactile sensing applications. These include diaphragm-type sensors, intrinsic fiber optic sensors and ablated cladding sensors [55],[56],[57]. A sensor developed by 
Kinotex $^{\mathrm{TM}}$ is based on the deformation of an optical integrating cavity, and has many of the advantages of previous sensors as well as being simple and robust [58].

Each Kinotex ${ }^{\mathrm{TM}}$ sensor is comprised of two optical fibers embedded in polymer foam. One optical fiber transmits light from a light emitting diode (LED) to an integrating cavity (the source fiber) and the other transmits light from the integrating cavity to a photodiode (the detector fiber). This sensor architecture can be seen in Figure 1. Most of the time, the integrating cavity is not a physical cavity, but instead is a virtual space in a uniform, transparent scattering medium [58]. An illumination source in a scattering medium creates a surrounding area of scattered energy, with a distance and medium dependant intensity [58]. The radiating energy has scattered energy $E_{S}$, original intensity $E_{o}$, characteristic scattering length of the medium $C_{s l}$, medium thickness $t$ and normalized optical thickness $t / C_{s l}$ as described by Equation 1 [58].

$$
E_{s}=E_{o}\left[1-e^{t / C_{s l}}\right]
$$

When pressure is applied, the medium is compressed and both the thickness and characteristic scattering length reduce proportionately [58]. The parameter of interest, $C_{s l}$, is measured by embedding an energy detecting fiber adjacent to the light source fiber in a way that allows the detecting fiber to sense scattered energy only [58]. Because the fibers are very small in comparison to the medium, the detecting fiber senses specifically the integrated intensity of the scattered light [58]. This intensity is integrated over a volume with radius of one characteristic scattering length $\left(C_{s l}\right)$, and an increase in energy intensity associated with applied pressure is able to be detected and converted to a voltage signal by a photodiode [58]. A number of these fiber optic sensors in an 
appropriately spaced array, embedded in a compressible uniform scattering medium can be used to generate voltage signals and determine the location, extent and direction of an applied force.

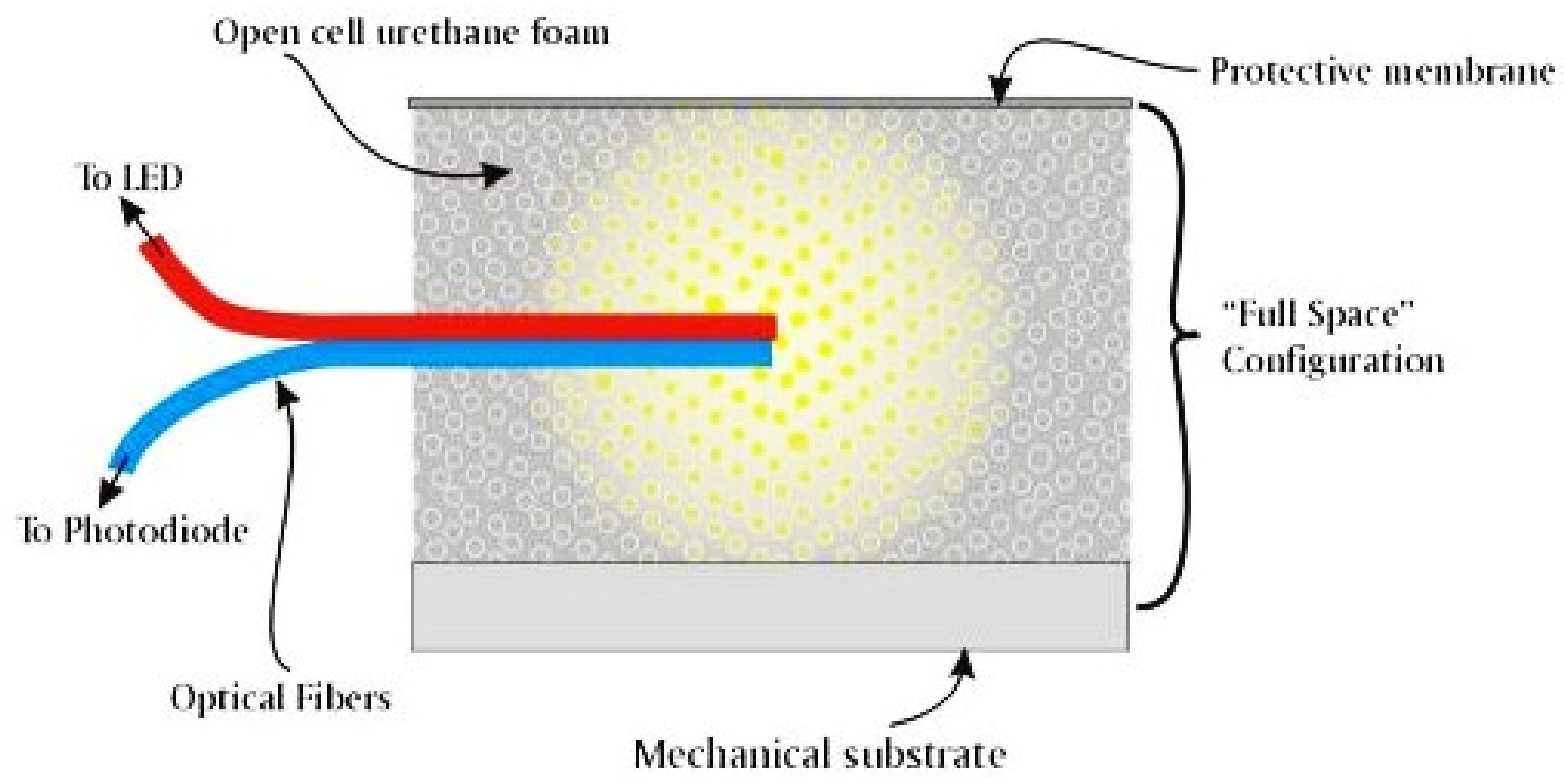

Figure 1: Kinotex ${ }^{\mathrm{TM}}$ sensor architecture

\subsection{Related Work}

This thesis builds upon work previously completed with Kinotex ${ }^{\mathrm{TM}}$ fiber optic sensors in the field of geriatrics, particularly in ubiquitous computing concerning the mobility of a subject.

Work completed by Nick Foubert at Carleton University in his Master's thesis, [59], aimed to determine first if a subject was sitting or lying in one of several orientations, and then if a subject was transitioning from lying to sitting while in bed. The body of work detailed the examination of support vector machines (SVMs), nearest 
neighbour $(\mathrm{NN})$ and k-nearest neighbour $(\mathrm{kNN})$ classifiers in the determination of one classifier that would distinguish between four different lying positions and two different sitting positions. While this thesis has a different system structure, different analysis and a different purpose, the aim is to detect similar orientations and so many of the same features are extracted and used in classification.

Other work completed by Amaya Arcelus at Carleton University in her PhD thesis, [60], examined several different algorithmic methods in the assessment of sit to stand transfers. Pressure images were used identify regions of interest, assess the symmetry of transfers and detect bouncing [61]. The center of pressure trajectories and duration measurements were calculated and used to detect mobility impairment and distinguish from the un-impaired [62]. This thesis again has a different structure and purpose, deals with more orientations and transfers, but builds upon elements of this work in the detection of similar orientations and transfers.

\subsection{Conclusions}

This chapter has discussed frailty in elderly patients, the HABAM tool, fiber optic pressure sensing and work completed with pressure sensing particular to the assessment of mobility in elderly patients, and relevant to this thesis. The proposed research aims to enhance and inform current HABAM assessments by introducing fiber optic based pressure sensitive mats and use them in conjunction with data processing to develop a system that automates in-bed HABAM assessments. 


\section{Chapter 3}

\section{Equipment and Experimentation}

The equipment used to gather data, the volunteer experimentation protocol and the organization of data is discussed in the following chapter sections.

\subsection{Introduction}

This chapter and the following chapters refer to certain areas of a hospital bed from a bird's eye perspective. The bird's eye schematic in Figure 2 puts these descriptive locations in context with the set-up of the room. Two sides of the hospital bed were placed flush against the wall; the right side of the bed, and the head of the bed. The terms 'Head' and 'Foot' refer to the areas indicated on the hospital bed, and are the areas where a patients head and feet would rest when lying supine on the bed. The left and right sides of the bed are the opposite of a patient's left and right side, as the referred orientation is from a bird's eye perspective. 


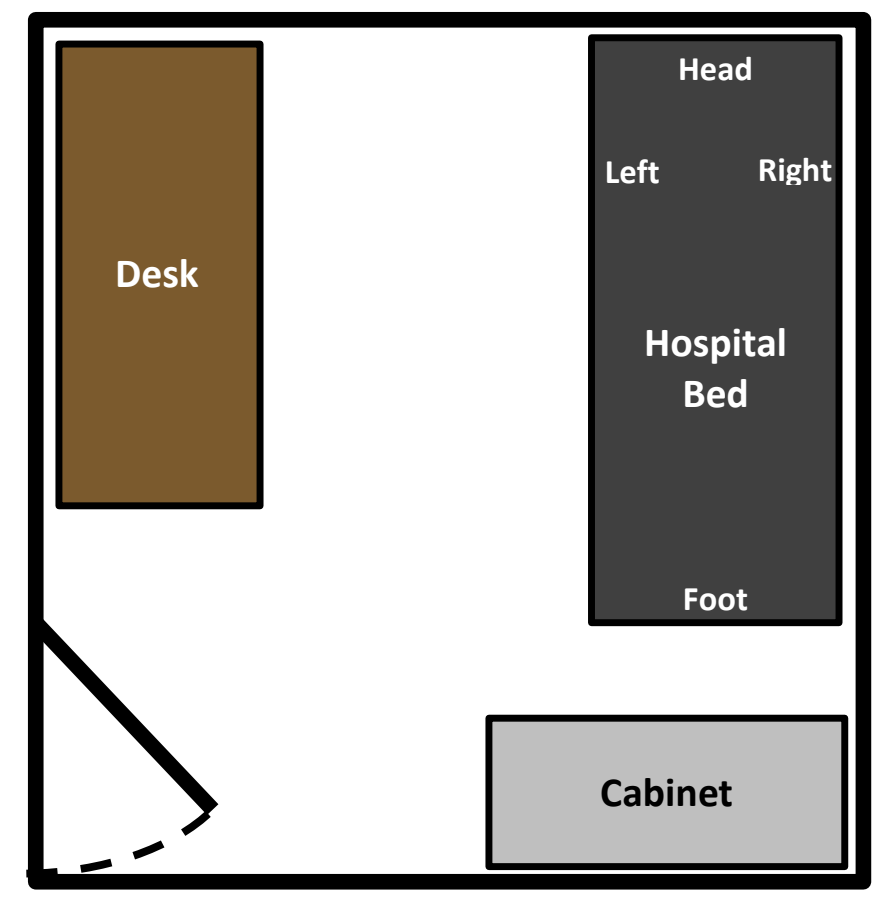

Figure 2: A reference schematic of the hospital bed in the experimentation room.

\subsection{Instrumentation}

The equipment used were inclusive of a standard laptop, a standard video camera, three pressure sensitive mats manufactured by S4 sensors (formerly Tactex Controls Inc.), and accompanying software. The mats were designed and built in accordance with UL 1069 standards (Hospital Signalling and Nurse Call Equipment) and were certified by Intertek. According to the United States Food and Drug Administration (FDA), the pressure mats fall under the least stringent classification because they: (a) do not come into direct contact with the patient, (b) do not utilize high voltage, and (c) do not provide any diagnosis. Failure or malfunction of any sensor will result in no danger to the original adverse health outcomes. 
Each pressure sensitive mat is approximately $80 \mathrm{~cm}$ long, $25 \mathrm{~cm}$ wide and consists of a 3 (width) by 8 (length) pressure sensor array embedded in polymer foam (approximately $1 \mathrm{~cm}$ high), then covered in medical grade plastic. The dimensioned mat is seen in Figure 3, where the mat head is a small, slightly raised area containing associated mat hardware. The mats are considered in this thesis as two dimensioned objects in which the rough spacing of the embedded sensors is depicted in Figure 4.

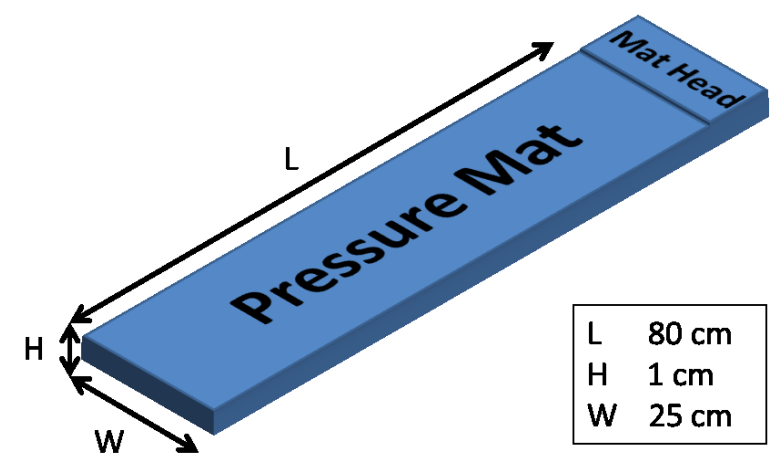

Figure 3: A single pressure mat and the dimensions.

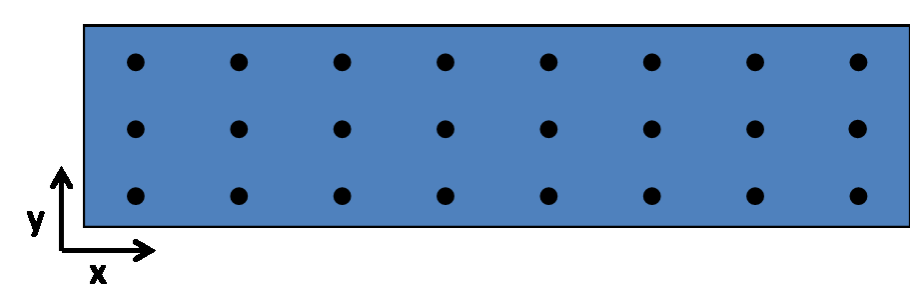

Figure 4: Spacing of the sensors within the pressure mat.

Each individual sensor is comprised of two optical fibers, a light emitting diode (LED), and an integrating cavity. A sensor measures pressure (in terms of voltage) by sending light from the LED through one optical fiber into the integrating cavity, and measuring the intensity of the light returning from the cavity through the other optical fiber to a photodiode [58]. Light intensity changes as pressure is applied, the photodiode 
reflects these changes in terms of voltage and this data is captured at sampling rate of 10Hz. The schematic of a single sensor is seen in Figure 5. These pressure sensors are discussed in more detail in Chapter 2 and more specific details on sensor behavior (such as saturation sensitivity, creep, and hysteresis) is detailed in [63].

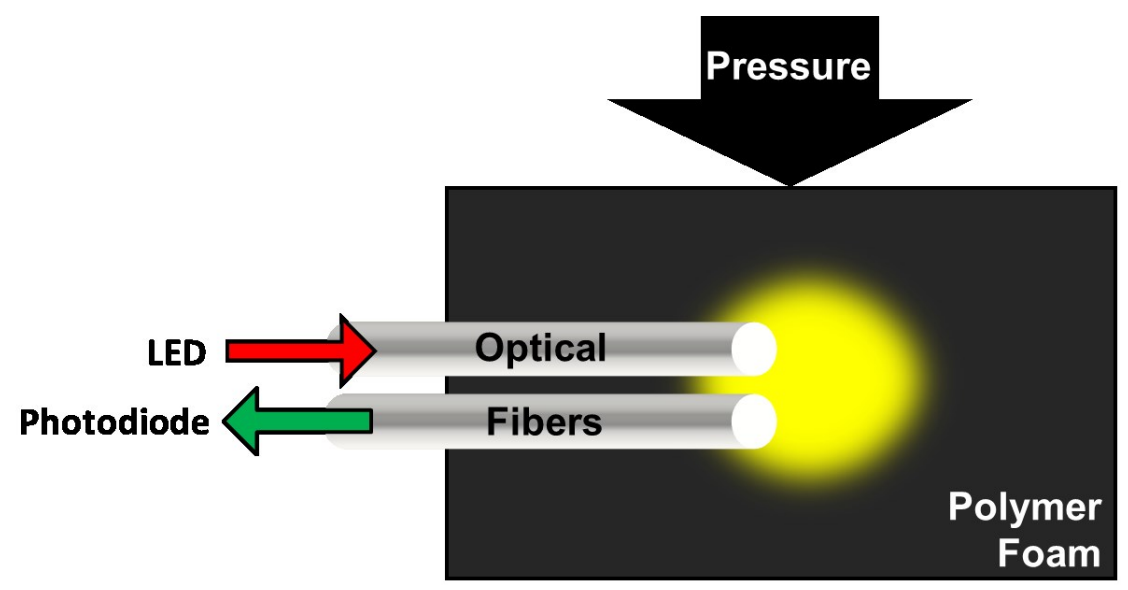

Figure 5: Schematic of a single pressure sensor embedded in polymer foam.

Each pressure sensitive mat consists of 24 sensors, and each sensor signal is carried to a separable box containing associated electronics and Bluetooth capability via a wire bundle. The wire bundle exits the mat from the right center of the mat head. Data generated by the photodiodes are in terms of voltage, and are sent via Bluetooth to a standard laptop where it is received by the mat software, transformed to nominal scoring (proportional to voltages) and stored in a comma separated values (csv) file. The video camera was used to document volunteer movements for reference purposes. 


\subsection{Equipment Set-Up}

The pressure mats were placed on the steel frame of a standard hospital bed, secured, and the accompanying standard hospital mattress was placed on top of the mats. The hospital bed frame is designed to fold in order to sit a patient up in bed, and so is constructed of four panels: one large panel supporting the back, two small panels at the sacrum, and an intermediate sized panel supporting the legs. Mat placement on the frame was dictated by the construction; each mat was oriented so that the length of the mat spanned the width of the frame. One mat was placed in the middle of each of the larger panels, and the last mat on top of the only panel at the sacrum that was large enough to support it. A bird's eye schematic of the bed frame and the placement of mats on the bed frame can be seen in Figure 6, where the darkened areas on the mats represent the mat heads.
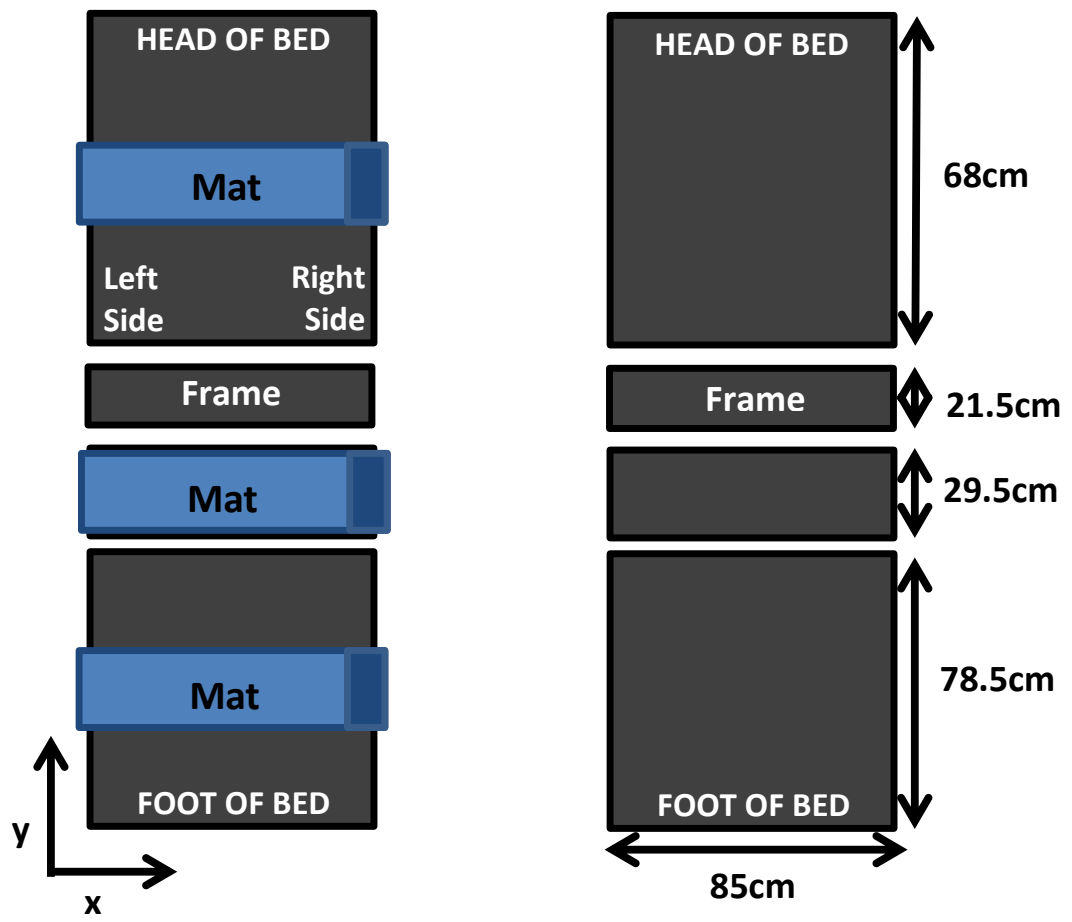

Figure 6: The bed fame dimensions and the placement of pressure mats on the frame. 
Each mat had one wire that connected the mat head to a removable Bluetooth transmitter box. For all experimentation, the bed was placed so that the right side of the bed was flush with the wall. The wires therefore extended out, underneath the mattress, down the right side of the bed and connected to respective Bluetooth transmitter boxes underneath the bed, on the floor.

The laptop was placed on a desk in the room (due to the wireless Bluetooth capability, the laptop could have been placed anywhere), and the video camera was held by hand in order to capture the volunteer movements for reference purposes.

\subsection{Experimentation}

Thirty healthy adult volunteers were recruited to determine if pressure sensitive mats can be used to generate a HABAM score. Volunteers were adults of any age who were not hospital patients and who provided consent to participate in the study.

Data were collected from the mattress alone as well as from 30 volunteers performing entirely in-bed enactments of HABAM scores. These scores were from two different categories on the HABAM form; the Transfers section and the Mobility section. From the Mobility section three scores were enacted: a score of 0 for 'Needs positioning in bed', a score of 4 for 'Positions self in bed', and a score of 7 for 'Lying-sitting independently'. From the Transfers section two scores were enacted: a score of 16 for 'Independent' and a score of 18 for 'Independent and Vigorous'. Each volunteer was asked to lie quietly, without moving, for 5 seconds before every movement. This allowed for the calculation of a baseline pressure reading. After lying quietly, each volunteer performed five separate movements (captured separately) that were enactments of 
different levels of ability representing these HABAM scores. The following sections describe these movements in more detail.

\subsubsection{Scenario 1}

Scenario 1 is the study name allocation for the enactment of a HABAM Mobility score of 0: 'Needs positioning in bed'. This term means that a patient is unable to lift supine pressure points off of the bed. These points include under the head, two scapulae, two at the sacrum region and two underneath the heels. This movement was therefore: lie quietly for 5 seconds, then move for approximately 20 seconds attempting (unsuccessfully) to roll to a side of the body without ever lifting a pressure point off the bed. The resulting movement resembled a supine person shifting weight without changing body orientation. A schematic of this movement is seen in Figure 7, where the red arrows represent the general directions pressure is applied, without lifting any supine pressure points.

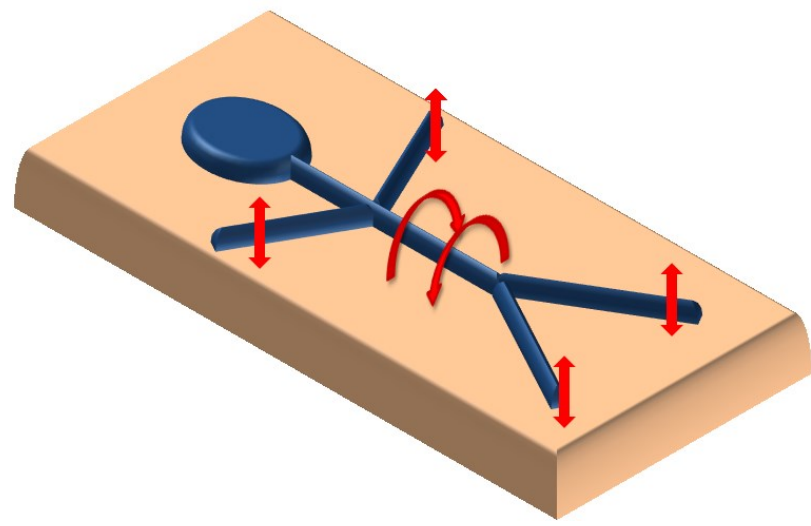

Figure 7: Schematic of Scenario 1 movements. 


\subsubsection{Scenario 2}

Scenario 2 is the study name allocation for the enactment of a HABAM Mobility score of 4: 'Positions self in bed'. This term means that the patient is able to lift supine pressure points off of the bed in order to re-position the body. This movement was therefore; lie quietly for 5 seconds, then for approximately 20 seconds roll from a supine position onto one side, back to a supine position and continue on the other side. The volunteers were asked to feign difficulty with most movements in order to simulate an older frail adult. The 'slow' rate at which volunteers were to roll was hard to regulate, so the frequency of rolling varied. The schematic in Figure 8 depicts the movements of a volunteer performing Scenario 2, where the large red arrows indicate movement from a supine position fully onto the right and/or left side of the body.

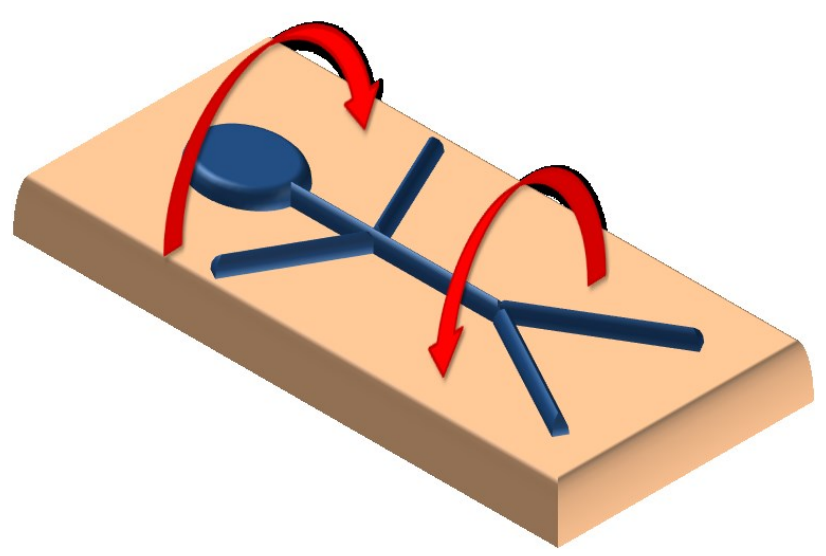

Figure 8: Schematic of Scenario 2 movements.

\subsubsection{Scenario 3}

Scenario 3 is the study name allocation for the enactment of a HABAM Mobility score of 7: 'Lying-sitting independently'. This term means that the patient is able to independently 
lift their upper body to a sitting position. This movement was therefore; lie quietly for 5 seconds, then over approximately 10 seconds, lift the upper body (allowing the use of elbows and hands) from a lying position into a sitting position. Again, difficulty was feigned and the 'slow' pace of volunteers was varied. The schematic in Figure 9 depicts the movements of a volunteer enacting Scenario 3 by transitioning from a supine lying position to a sitting position.

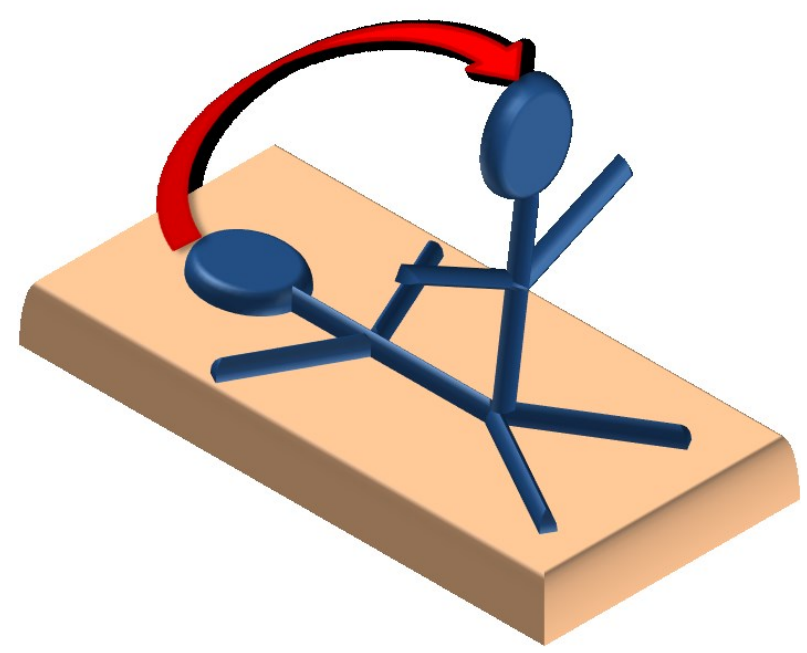

Figure 9: Schematic of Scenario 3 movements.

\subsubsection{Scenario 4}

Scenario 4 is the study name allocation for the enactment of a HABAM Transfers score of 14: 'Independent but slow'. This term means that a patient is capable of independently transferring from a sitting position to a standing position. A sitting position for this scenario means sitting on the left edge of the bed with the legs extending out over the left side of the bed and bent at the knees with the feet on the ground. This movement was therefore: sit on the edge of the bed for 5 seconds and transfer slowly from a sitting to a 
standing position in approximately 10 seconds while feigning difficulty (the use of hands for support on either side of the body was encouraged to better simulate an older frail patient). The length of time to stand was chosen as advised by medical staff in the Geriatric ward to better simulate elderly in-patients. The schematic in Figure 10 depicts a volunteer transitioning from the described sitting position to a standing position.

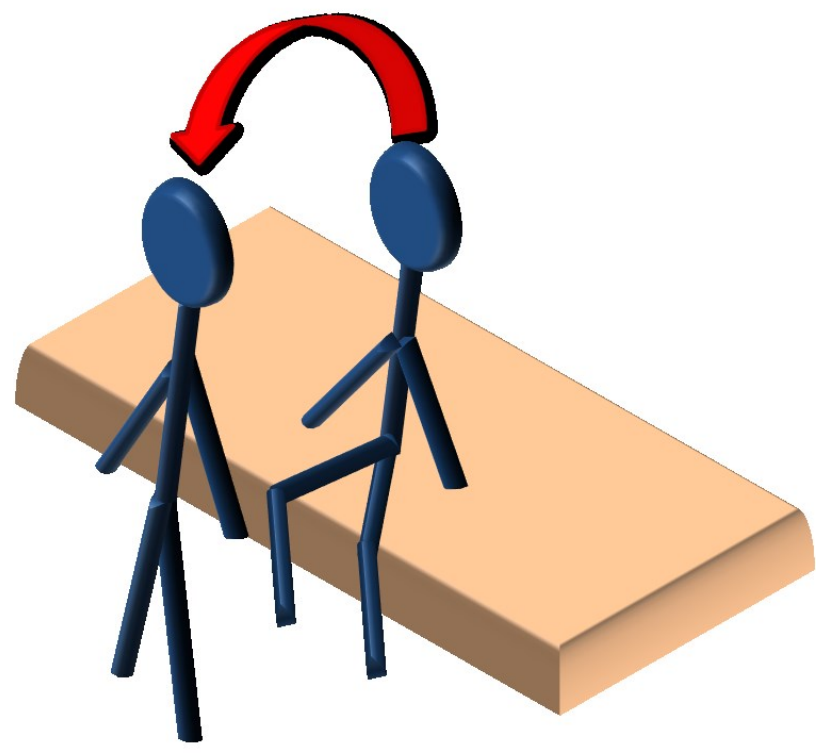

Figure 10: Schematic of Scenario 4 movements.

\subsubsection{Scenario 5}

Scenario 5 is the study name allocation for the enactment of a HABAM Transfers score of 18: 'Independent and vigorous'. This term means that a patient has no impairment in transitioning from a sitting position to a standing position. This movement was therefore; sit on the edge of the bed for 5 seconds and transfer vigorously from a sit to a stand position in approximately 1-2 seconds. The sitting position is the same sitting position described in Section 3.4.4, and depicted in Figure 10. The approximated time to stand is the length of time on average that a non-patient (or an able in-patient) takes to stand. 


\subsection{Data Organization and Calibration}

Data was collected from thirty volunteers, each during a separate session. Each session resulted in 6 comma separated values (csv) data files and 5 video files. One csv file contains data from the mattress alone, gathered before respective volunteers performed any enactments. The other five csv files contain data from each of the five scenarios, which are accompanied by a video file primarily captured for reference purposes. The following sections detail how the data were organized and calibrated.

\subsubsection{Organization of Data}

The data collected are output by the mat associated software in terms of nominal scores proportional to the pressure applied. The sensors are designed to collect data at a frequency of $10 \mathrm{~Hz}$, outputting a nominal score (proportional to the pressure applied) every 0.1 seconds for a total of 720 data points every second. For the purposes of this thesis, to facilitate later analysis, the sensor outputs were considered on an individual and summed basis. The notation used in this section, and throughout this thesis is described in Table 1.

Table 1: Key terms and the designated notations

\begin{tabular}{|c|c|c|}
\hline Key Terms & Notation & Range of Values \\
\hline \hline Participant Number & $\mathrm{p}$ & $1: 30$ \\
\hline Scenario Number & $\mathrm{sc}$ & $1: 5$ \\
\hline Sensor Row & $\mathrm{r}$ & $1: 9$ \\
\hline Sensor Column & $\mathrm{c}$ & $1: 8$ \\
\hline Time (seconds) & $\mathrm{t}$ & Scenario Dependent \\
\hline
\end{tabular}


The sensors were first considered to be in a 9 row by 8 column array as indicated in Figure 11. The individual sensor data was collected in a series of arrays, representing the participant number, the scenario number, the sensor row indices and the sensor column indices. This structure is described in Equation 2. Each cell within the primary array represents the participant and scenario. Each cell within the secondary array represents a sensor and contains all data generated by that sensor for that participant and scenario. This resulted in one 9 by 8 (by the total number of frames) data array for each participant and each scenario, essentially creating a 'snapshot' of respective mat data at every point in time. This data was named ISD, for Individual Sensor Data as seen in Equation 2.

$$
\operatorname{ISD}\{p, s c\}\{r, c\}(t)
$$

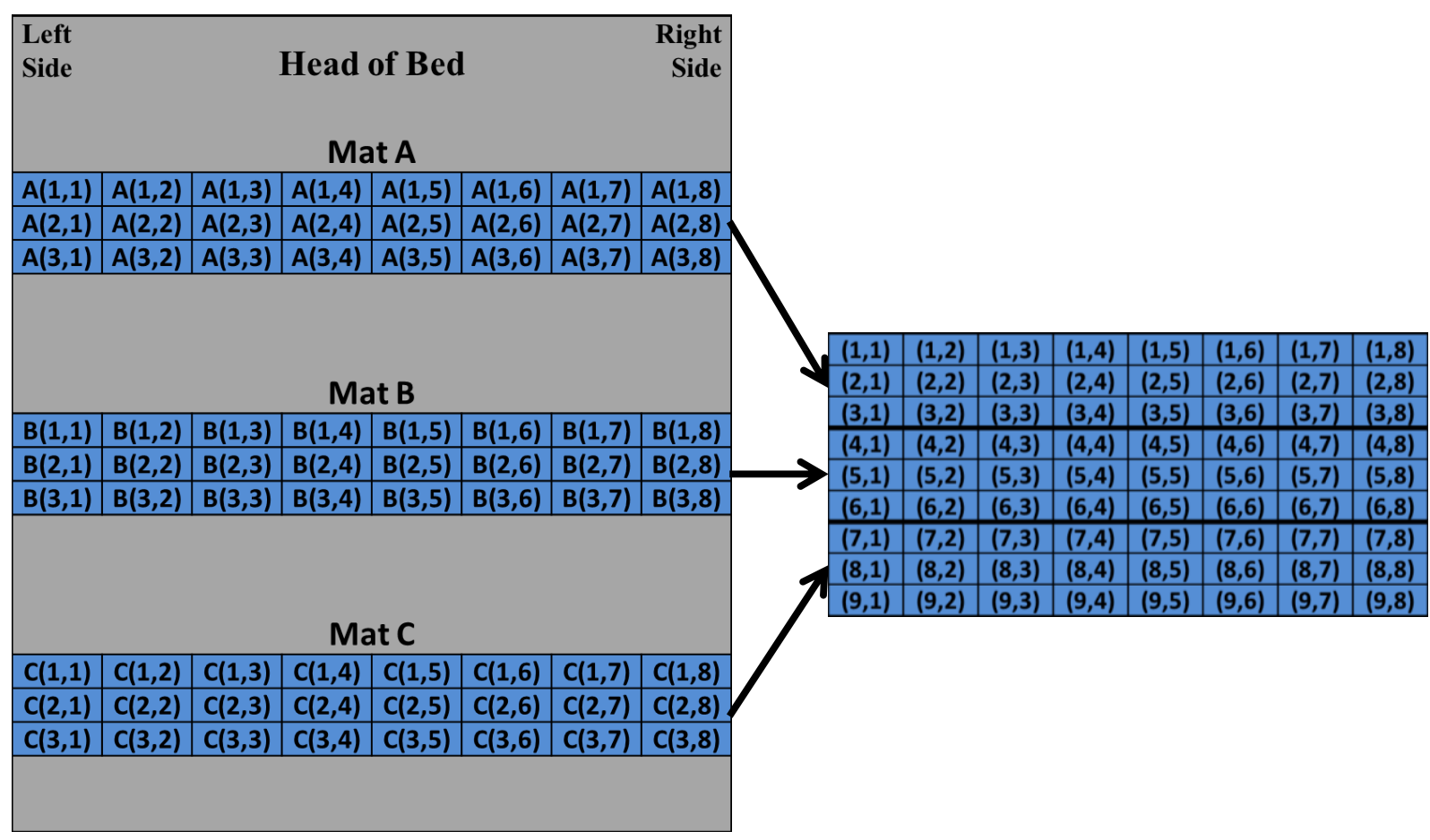

Figure 11: Consideration of mats as one data array 
The second structure was in terms of sensor data sums. These sums were of each individual row and column of the sensor array depicted in Figure 11 and are functions of three variables: participant number, scenario number and time. The row sums are depicted in Figure 12, where each mat is given a label (A, B and C), and each sensor has been given indices indicating its location within the sensor array. The grey area behind the mats represents the hospital bed frame. Areas on the mats of the same color represent the sensor sums; at every point in time all sensor values within areas of the same color were summed, for a total of nine row sum values every 0.1 seconds (for one participant and one scenario). These sums are labelled R1, R2, R3, etc. as seen in Figure 12 and as listed in Table 3.

The column sums are depicted in Figure 13, where again each mat is labeled, and each sensor location within the embedded array is indicated within each mat. The colored areas span the length of the bed instead of the width, and again represent the sensor sums. At every point in time each sensor value within each colored area was summed, for a total of eight column sum values every 0.1 seconds (for one participant and one scenario). These sums are referred to as $\mathrm{C} 1, \mathrm{C} 2, \mathrm{C} 3$, etc. as listed in Table 3. These column and row sums were calculated for each participant performing each scenario at every point in time, outputting a total of $150 \mathrm{csv}$ files. 


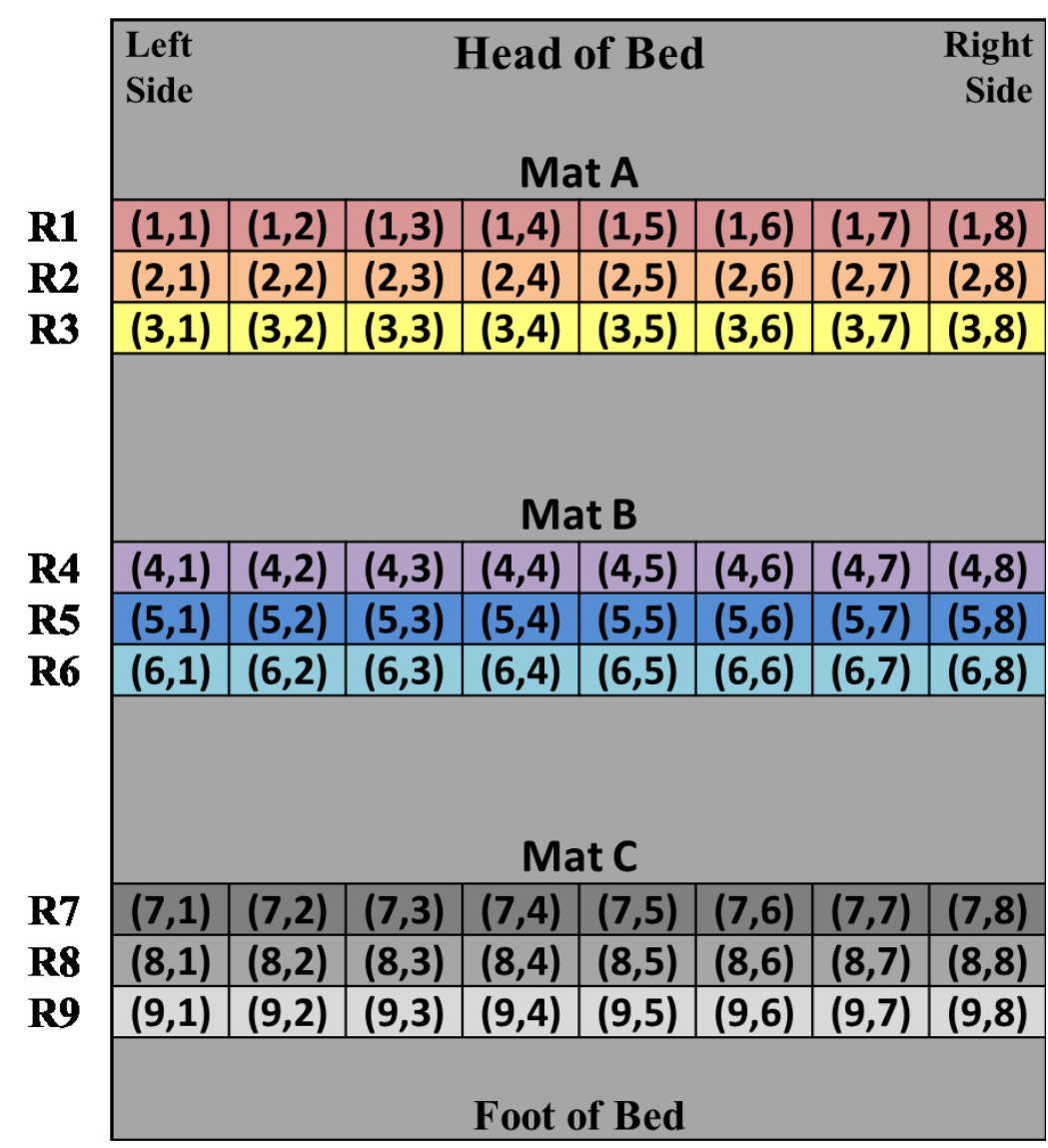

Figure 12: Individual row sums

Table 2: Row sum acronyms

\begin{tabular}{|c|c|}
\hline Variable & $\begin{array}{c}\text { Variable } \\
\text { Acronym }\end{array}$ \\
\hline Sum Row 1 & R1 \\
\hline Sum Row 2 & R2 \\
\hline Sum Row 3 & R3 \\
\hline Sum Row 4 & R4 \\
\hline Sum Row 5 & R5 \\
\hline Sum Row 6 & R6 \\
\hline Sum Row 7 & R7 \\
\hline Sum Row 8 & R8 \\
\hline Sum Row 9 & R9 \\
\hline
\end{tabular}




\begin{tabular}{|c|c|c|c|c|c|c|c|}
\hline $\begin{array}{l}\text { Left } \\
\text { Side }\end{array}$ & \multicolumn{6}{|c|}{ Head of Bed } & $\begin{array}{l}\text { Right } \\
\text { Side }\end{array}$ \\
\hline$(1,1)$ & $(1,2)$ & $(1,3)$ & $(1,4)$ & $(1,5)$ & $(1,6)$ & $(1,7)$ & $(1,8)$ \\
\hline$(2,1)$ & $(2,2)$ & $(2,3)$ & $(2,4)$ & $(2,5)$ & $(2,6)$ & $(2,7)$ & $(2,8)$ \\
\hline$(3,1)$ & $(3,2)$ & $(3,3)$ & $(3,4)$ & $(3,5)$ & $(3,6)$ & $(3,7)$ & $(3,8)$ \\
\hline$(4,1)$ & $(4,2)$ & $(4,3)$ & $(4,4)$ & $(4,5)$ & $(4,6)$ & $(4,7)$ & $(4,8)$ \\
\hline$(5,1)$ & $(5,2)$ & $(5,3)$ & $(5,4)$ & $(5,5)$ & $(5,6)$ & $(5,7)$ & $(5,8)$ \\
\hline$(6,1)$ & $(6,2)$ & $(6,3)$ & $(6,4)$ & $(6,5)$ & $(6,6)$ & $(6,7)$ & $(6,8)$ \\
\hline$(7,1)$ & $(7,2)$ & $(7,3)$ & $(7,4)$ & $(7,5)$ & $(7,6)$ & $(7,7)$ & $(7,8)$ \\
\hline$(8,1)$ & $(8,2)$ & $(8,3)$ & $(8,4)$ & $(8,5)$ & $(8,6)$ & $(8,7)$ & $(8,8)$ \\
\hline$(9,1)$ & $(9,2)$ & $(9,3)$ & $(9,4)$ & $(9,5)$ & $(9,6)$ & $(9,7)$ & $(9,8)$ \\
\hline C1 & C2 & C3 & $\begin{array}{c}\text { C4 } \\
\text { Foot }\end{array}$ & $\begin{array}{l}\text { C5 } \\
\text { f Bed }\end{array}$ & C6 & C7 & C8 \\
\hline
\end{tabular}

Figure 13: Individual column sums

Table 3: Column sum acronyms

\begin{tabular}{|c|c|}
\hline Variable & $\begin{array}{c}\text { Variable } \\
\text { Acronym }\end{array}$ \\
\hline \hline Sum Column 1 & $\mathrm{C} 1$ \\
\hline Sum Column 2 & $\mathrm{C} 2$ \\
\hline Sum Column 3 & $\mathrm{C} 3$ \\
\hline Sum Column 4 & $\mathrm{C} 4$ \\
\hline Sum Column 5 & $\mathrm{C} 5$ \\
\hline Sum Column 6 & $\mathrm{C} 6$ \\
\hline Sum Column 7 & $\mathrm{C} 7$ \\
\hline Sum Column 8 & $\mathrm{C} 8$ \\
\hline
\end{tabular}




\subsubsection{Data Calibration}

Data calibration began by incorporating initial start times for each scenario. Each of thirty participants performed five scenarios. These scenarios were captured by one researcher, who operated the laptop and the video camera simultaneously. The start times for data collection and video footage were therefore slightly staggered. This stagger was orchestrated; the sensor data collection was started before a pre-determined scenario start time at which the video data collection began. Data was aligned by identifying the predetermined scenario start time and deleting all preceding sensor data.

The second stage of calibration was to eliminate the weight of the mattress from data representing participants performing movements on a hospital bed. Ideally, when the participant exits the bed, there is no longer any pressure exerted and the system developed in this thesis would reflect this zero pressure. Without eliminating the weight of the mattress, the system will never reflect zero pressure. For this reason, data was first calibrated before further analysis.

Before each session with a different participant, data of the mattress alone was captured. This was simply a couple seconds worth of data captured of the pressure the hospital mattress exerted on each sensor, before the participant entered the bed. This data was structured as in Section 3.5.1; on both an individual sensor basis and in terms of summed rows and columns. Individual sensor data, summed row sensor data and summed column sensor data generated by the mattress was averaged over time to obtain the pressure exerted by the mattress on each sensor (in terms of nominal sores). These average pressures were subtracted from respective sum and individual sensor data for 
every enactment by each volunteer. The equations 3 and 4 describe the calculations for obtaining the Calibrated Individual Sensor Data, where $\operatorname{Mattress} A v\{p, s c\}(r, c)$ is the described average pressure of the mattress on each sensor and $\operatorname{ISD}\{p, s c\}\{r, c\}(t)$ is the individual sensor data described in in Section 3.5.1. The equations 5 and 6 describe the calculations for obtaining the Calibrated Summed Data (Sum of Row 1 was used for illustration), where $R 1 a v(p, s c)$ is the described average pressure of the mattress on the sensors in Row 1 and the notation for the calibrated row and column sums changes from upper case to lower case. I.e. $R 1\{p, s c\}(t)$ becomes $r 1\{p, s c\}(t)$ once calibrated. Table 4 indicates the new calibrated variable names and acronyms.

Table 4: Calibrated data variables.

\begin{tabular}{|c|c|}
\hline Calibrated Variable & $\begin{array}{c}\text { Variable } \\
\text { Acronym }\end{array}$ \\
\hline \hline Calibrated Individual Sensor Data & CISD \\
\hline Calibrated Row Sum & $\mathrm{r} 1, \mathrm{r} 2$, etc. \\
\hline Calibrated Column Sum & $\mathrm{c} 1, \mathrm{c} 2$, etc. \\
\hline
\end{tabular}

$$
\begin{gathered}
\text { MattressAv }\{p, s c\}(r, c)=\frac{\sum_{t} \text { Mattressav }\{p, s c\}\{r, c\}(t)}{t} \\
C I S D\{p, s c\}\{r, c\}(t)=I S D\{p, s c\}\{r, c\}(t)-\operatorname{Mattress} A v\{p, s c\}(r, c) \\
R 1 a v(p, s c)=\frac{\sum_{t} R 1\{p, s c\}(t)}{t} \\
r 1\{p, s c\}(t)=R 1\{p, s c\}(t)-R 1 a v(p, s c)
\end{gathered}
$$




\section{Chapter 4}

\section{Algorithmic System Set-Up}

This chapter describes the set up of the algorithmic system detailed in this thesis.

\subsection{Introduction}

The system described in this chapter aims to take raw, unidentified data and determine which of five HABAM scenarios the data represents. Scenarios 1 to 5 are described in detail in Section 3.4, and inclusive of: HABAM Mobility score of 0: 'Needs positioning in bed', HABAM Mobility score of 4: 'Positions self in bed', HABAM Mobility score of 7: 'Lying-sitting independently', HABAM Transfers score of 14: 'Independent but slow', HABAM Transfers score of 18: 'Independent and Vigorous', respectively.

\subsection{System Set-Up}

The algorithmic set-up was in the form of a decision tree, seen in Figure 14. The system was broken into three stages: a primary stage, Determination of Initial Posture (DIP), and two secondary stages, Distinction Between Lying Scenarios (DBLS) and Distinction Between Sitting Scenarios (DBSS). This was designed this way in order to explore different methods of pattern recognition better suited to certain orientations and movements, and also to create the simplest, most robust design able to distinguish 
between the HABAM enactments in question, with the potential to incorporate additional engineering solutions to improve the HABAM tool.

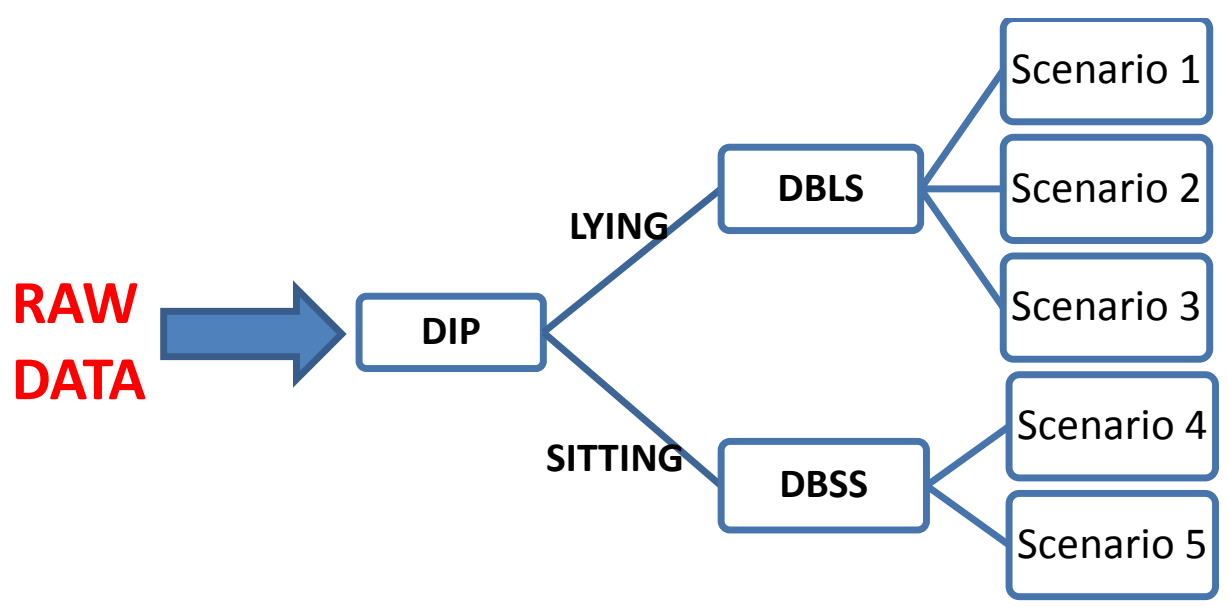

Figure 14: System tree describing the decision process for the entire algorithmic system.

The first stage, DIP, uses two different classifiers to determine the best method for identifying the initial position of the participant; a supine lying position, or a sitting position at the left center edge of the hospital bed. Relevant features were extracted and two classifiers analyzed the features for performance. The best performing features were further tested by adding Gaussian noise and again, the best performing features were used with the best performing classifier to create a final classification model. If the data in question was determined to have an initial lying position, this data was passed to a secondary stage, DBLS. If the initial position was determined to be sitting, then the data was passed to secondary stage DBSS.

The secondary stage, DBLS takes data from DIP and determines if the data in question represents scenarios 1,2 or 3 . These scenarios involve participants beginning in a supine lying position, and: attempting, but unsuccessfully relieving pressure points (Scenario 1), attempting and successfully relieving pressure points (Scenario 2) and 
transferring to sitting position (Scenario 3). The main method in this stage was to identify regions of interest, calculate a starting, baseline pressure value for these regions, and determine the percentage pressure change over time. DBLS was again separated into two stages, the first stage aimed to identify Scenario 3 by calculating the percentage pressure change in the mat at the head of the bed. If the data did not represent Scenario 3, it was passed to the second stage, which determined if the data represented Scenario 1 or Scenario 2. This was done by grouping the sensors into regions of interest representing pressure points underneath a supine participant, then monitoring percentage pressure change and identifying instances at which two or more sensors in the same area had been relieved of pressure. If no sensors were relieved, the data was determined to represent Scenario 1. If the described incidences occurred, the data was determined to represent Scenario 2.

The secondary stage, DBSS takes data from DIP and determines if the data in question represents Scenario 4 or Scenario 5. These scenarios involve participants beginning in a sitting position and transferring to a standing position slowly (Scenario 4), as well as transferring to a standing position vigorously (Scenario 5). This was done by mapping the center of pressure over time and analyzing the path this center of pressure travelled. Features were extracted and two classifiers were used to analyze features on an individual basis, and then the best performing features were subjected to added noise before final determination of features and type of classifier in a final model.

The first stage, DIP, is discussed in detail in Chapter 5, DBLS is discussed in Chapter 6, DBSS is discussed in Chapter 7 and the results of the entire system as well as final conclusions are discussed in Chapter 8. 


\section{Chapter 5}

\section{Determination of Initial Posture}

This chapter details the first stage of the algorithmic system; Determination of Initial Posture (DIP).

\subsection{Introduction}

Determination of Initial Posture (DIP) is briefly described in Chapter 4 and depicted in Figure 15. DIP aims to take raw, unidentified data gathered from three pressure sensitive mats of a subject on a hospital mattress and determine whether or not the subject's initial position is lying or sitting. More specifically whether the subject is in a supine lying orientation as seen in Figure 16, or sitting on the center left edge of the bed with their feet on the ground as seen in Figure 17. The output of DIP is binary, indicating either a lying or sitting position and determines which subsequent subsystem data is passed to, as seen in Figure 15. 


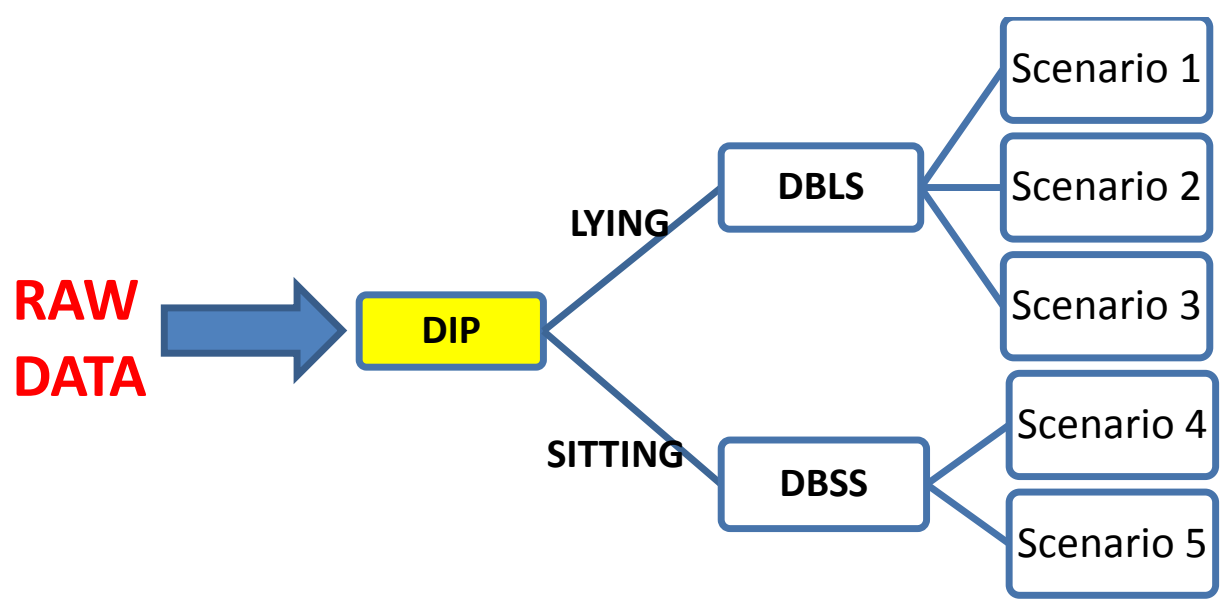

Figure 15: System tree describing the decision process for DIP as it applies to the whole system.

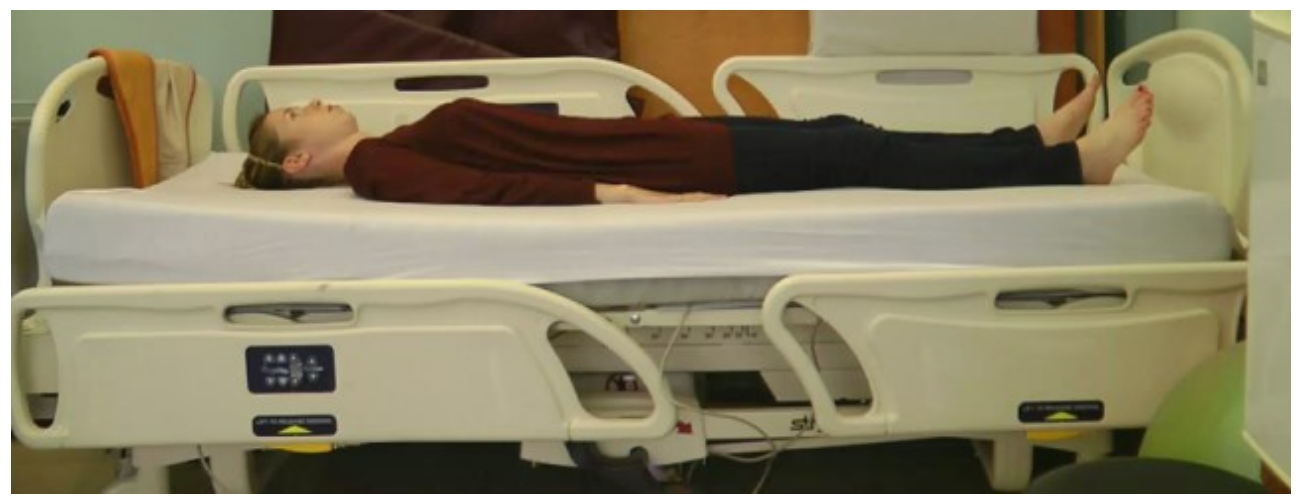

Figure 16: A subject in a supine lying position.

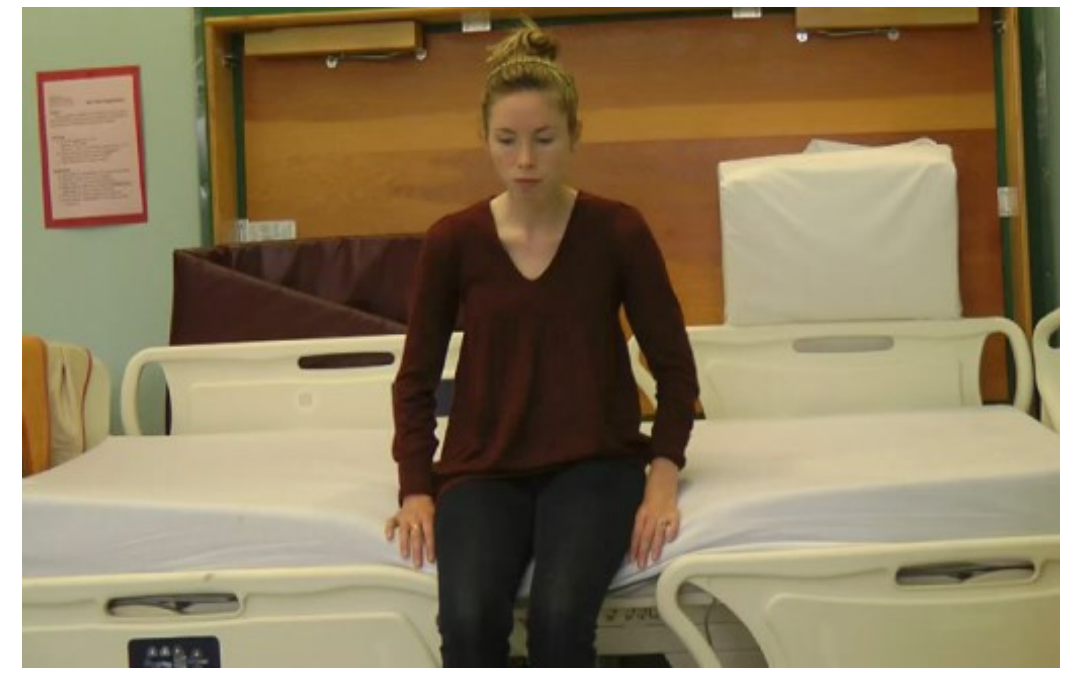

Figure 17: A subject in a sitting position on the left edge of the bed. 
This stage was designed by first extracting features from calibrated data, evaluating the performance of these features using an appropriate classifier, and building a final classifier using the best performing features. This chapter builds on work completed by Nick Foubert in his thesis [59], which aimed to determine first if a subject was sitting or lying in one of six orientations, and then if that subject was transitioning from lying to sitting while in bed. One classifier was used to distinguish between two sitting positions and four lying positions. In this chapter, DIP has a similar yet simplified goal of using one classifier to determine between two initial subject positions: supine lying and sitting on the left bed edge. As this is an assessment of a starting position (after which a movement follows), only data within the first 4 seconds are of interest to this subsystem. These first 5 seconds is the time during which volunteers were instructed to sit or lie quietly; 4 seconds was chosen to account for subject error.

\subsection{Feature Extraction}

All volunteers enacted scenarios on a hospital bed placed on top of three pressure sensitive mats spaced as in Figure 6, Section 3.3. The hospital mattress causes subject weight to spread across the mats (affect more sensors) more so than without the mattress. The calibration detailed in Section 3.5.2 aims to eliminate the weight of the mattress, but does not eliminate the spread. For this reason, measures of occupancy and spread were chosen in favor of such methods as edge detection. As this system is designed for people of varying heights and weights, the features chosen were as subject independent as possible. Perfect subject independence is difficult, as bed occupancy and spread is inherently dependant on subject size and weight. Direct reliance on these variables was 
avoided by choosing features that were not simply conversions of patient weight. For example, a feature that simply observes sensor scores or sums of sensor scores may change drastically from person to person as sensor scores are pressure conversions, and pressure depends on patient size and weight. In this system, a feature that allows for drastic changes based on weight can make classification difficult. Subject dependence was therefore avoided by using percentages, areas and measures of central pressure.

The following sections describe the features designed and used for the analysis of data in Subsystem 1. Table 5 summarizes the features examined and the respective chapter sections, feature acronyms and equation reference numbers.

Table 5: Summary of features.

\begin{tabular}{|c|c|c|c|}
\hline Feature & $\begin{array}{c}\text { Feature } \\
\text { Acronym }\end{array}$ & $\begin{array}{c}\text { Chapter } \\
\text { Section }\end{array}$ & $\begin{array}{c}\text { Equation } \\
\text { Numbers }\end{array}$ \\
\hline Percentage of Active Sensors & PAS & 5.2 .1 & $7-12$ \\
\hline Longitudinal Center of Pressure & LonCP & 5.2 .2 & $13-14$ \\
\hline Latitudinal Center of Pressure & LatCP & 5.2 .3 & 15 \\
\hline Longitudinal Variance & LonV & 5.2 .4 & $16-17$ \\
\hline Latitudinal Variance & LatV & 5.2 .5 & $18-19$ \\
\hline Central Left Ratio & CLR & 5.2 .6 & 20 \\
\hline Quarter Left Ratio & QLR & 5.2 .7 & 21 \\
\hline
\end{tabular}

\subsubsection{PAS: Percentage of Active Sensors}

This feature is an adapted version of the feature: Number of Active Sensors (NAS), used in previous work by Nick Foubert [59]. The NAS and PAS allows for a measure of the bed space occupied at any one point in time, where PAS is better able to account for different mats or sensors, should new materials be of interest. NAS was calculated by summing the number of sensors exceeding a sensor score threshold, and PAS was calculated by converting this number to a percentage. In [59], the threshold was 
considered to be a sensor score of zero, or in other words if a sensor detected any pressure at all, it was considered to be active. Here, the zero score threshold is not an effective limit. The pressure distribution effects of the mattress create low level noise in sensor score signals. This is illustrated using a 'snapshot' image of one example participant lying supine on the bed, created by averaging the first four seconds of data from each sensor. Figure 18 depicts the snapshot image of the example participant in two different ways: by depicting respective sensor scores on a color scale representative of the output values (Figure 18, right subplot), and in a simpler white and black representation of active, weight bearing sensors and non-active, non-weight bearing sensors, respectively (Figure 18, left subplot). Each sensor is represented by a square indicating the general location of a sensor and the average sensor score. Spacing between sensors and mats in the figure is proportional to the physical spacing of the sensor and mats on the hospital bed. Areas of black represent zero occupancy, including the physical space between mats in which there are no sensors. The left subplot depicts the active sensors (in white) when the threshold is a sensor score of zero. There are many sensors close to the bed edges generating relatively low scores (below 200), yet are considered to be active. This is likely the result of pressure spread due to the hospital mattress. Figure 18 demonstrates through observation that at a sensor score threshold of zero, low level noise created by the spread of data causes the features NAS and PAS to become uninformative. 

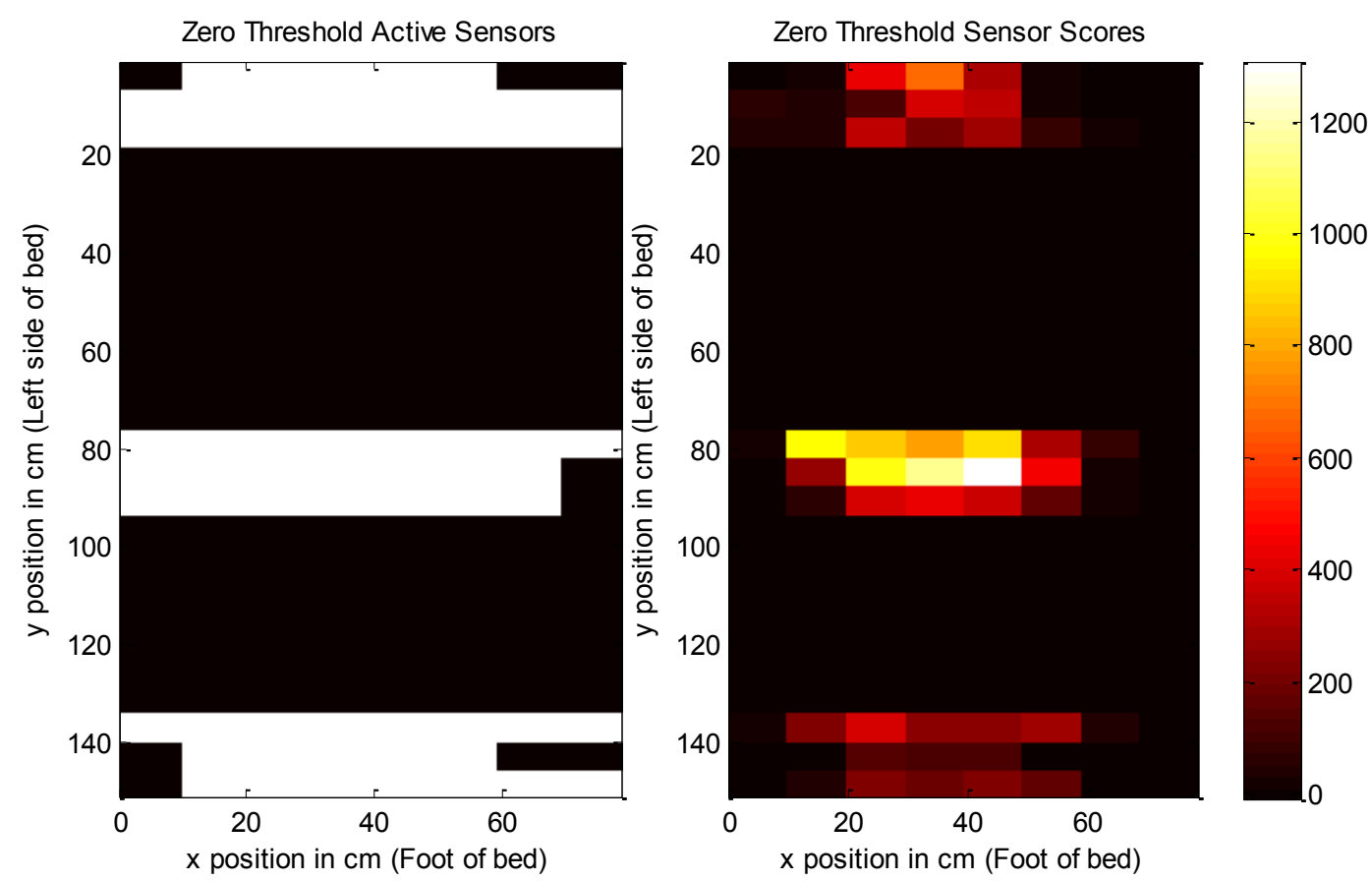

Score

Figure 18: Average still data from Participant 5 lying supine on the bed with a zero score threshold.

Non-zero thresholds are therefore needed to distinguish properly between active and non-active sensors. This threshold was calculated on an individual subject basis using an iterative process. For each subject, a snapshot of the initial position was created by averaging calibrated data (calibration is described in Section 3.5.2) for each sensor across the first 4 seconds (40 frames) of data; the time during which the subject was sitting or lying quietly. This data was collected for each participant and each scenario in a 9x8 matrix, where each cell represents the average sensor score of a respective sensor. This calculation is referred to in this section as the Initial Position Snapshot, $\operatorname{IPS}\{p, s c\}(r, c)$.

The average PAS during the first four seconds was then calculated for all scenarios and subjects as seen in equations 7 to 9 below, where $\operatorname{IPS}\{p, s c\}(r, c)$ represents the average sensor scores for respective sensors, the summation is over the total number of sensors (72 in a 9x8 array) and $A(\operatorname{IPS}\{p, s c\}(r, c))$ is a step function that 
is equivalent to 1 if the sensor score is greater than the threshold, and 0 if the sensor score is below the threshold. The threshold value was varied from 0 to the maximum average sensor score value for each subject, to observe the full range of possible threshold values.

$$
\begin{aligned}
& \operatorname{PAS}\{p, s c\}(m)=\frac{\sum_{r=1}^{9} \sum_{c=1}^{8} A(I P S\{p, s c\}(r, c))}{72} \\
& A(I P S\{p, s c\}(r, c))=\left\{\begin{array}{rr}
1 & \text { if } \operatorname{IPS}\{p, s c\}(r, c)>\operatorname{threshold}\{p, s c\}(m) \\
0 & \text { otherwise }
\end{array}\right. \\
& \operatorname{threshold}\{p, s c\}(m)=1: m, \quad \text { where } m=\max (\operatorname{IPS}\{p, s c\}(r, c))
\end{aligned}
$$

For each subject, and each scenario, there was a resulting PAS data point for every possible corresponding threshold. This data was then separated into two groups: one group for those scenarios with an initial lying position, and another group for those scenarios with an initial sitting position. Within these groups, PAS data was averaged at every threshold value and compared. An example plot of the resulting group average PAS signals for the example participant is seen in Figure 19. 


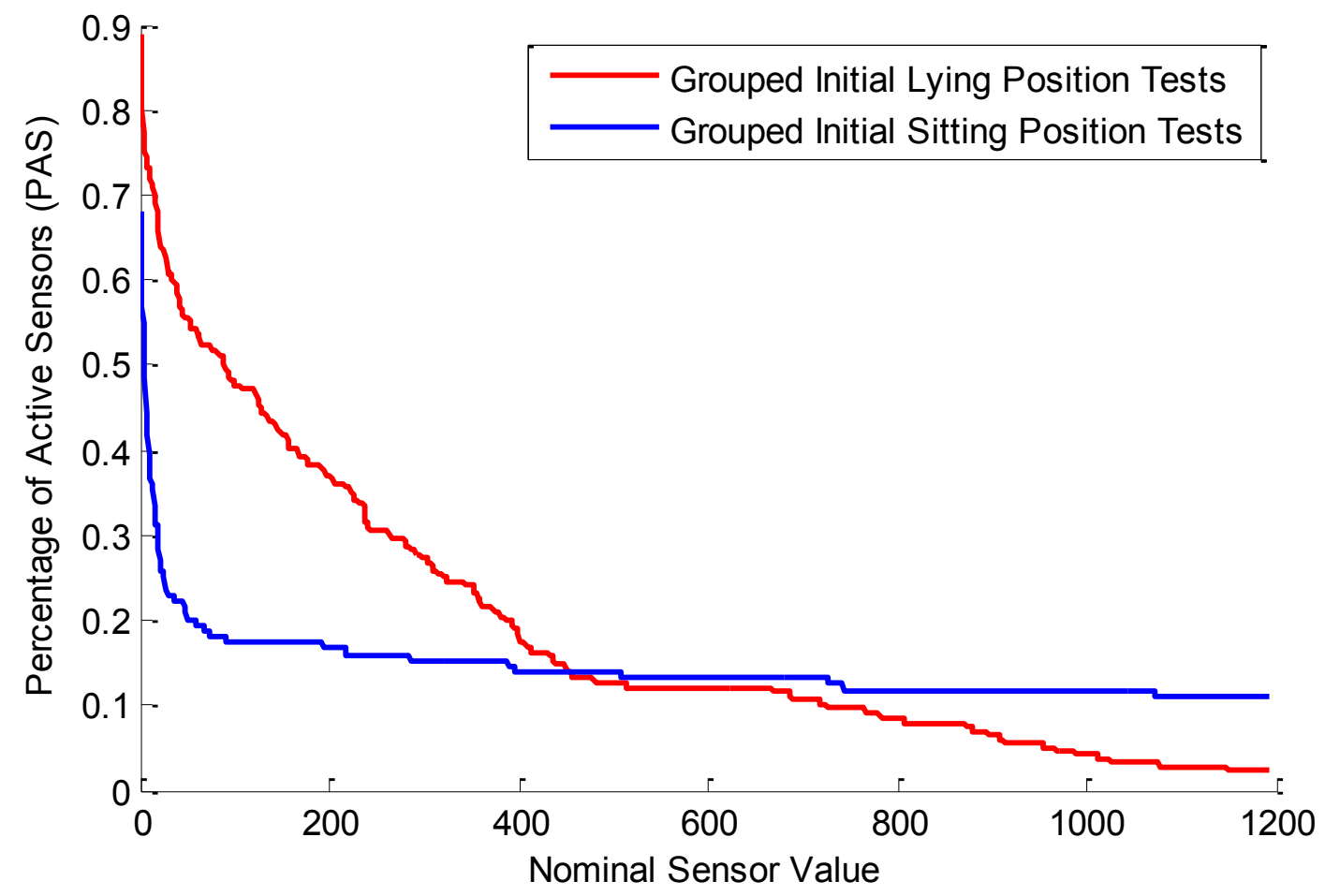

Figure 19: PAS at varying threshold values for grouped tests

The difference between these two PAS signals was calculated and the maximum value peak was allocated as the participant threshold. Grouped average PAS and a PAS difference signal with the maximum peak for Participant 5 is illustrated in Figure 20. 


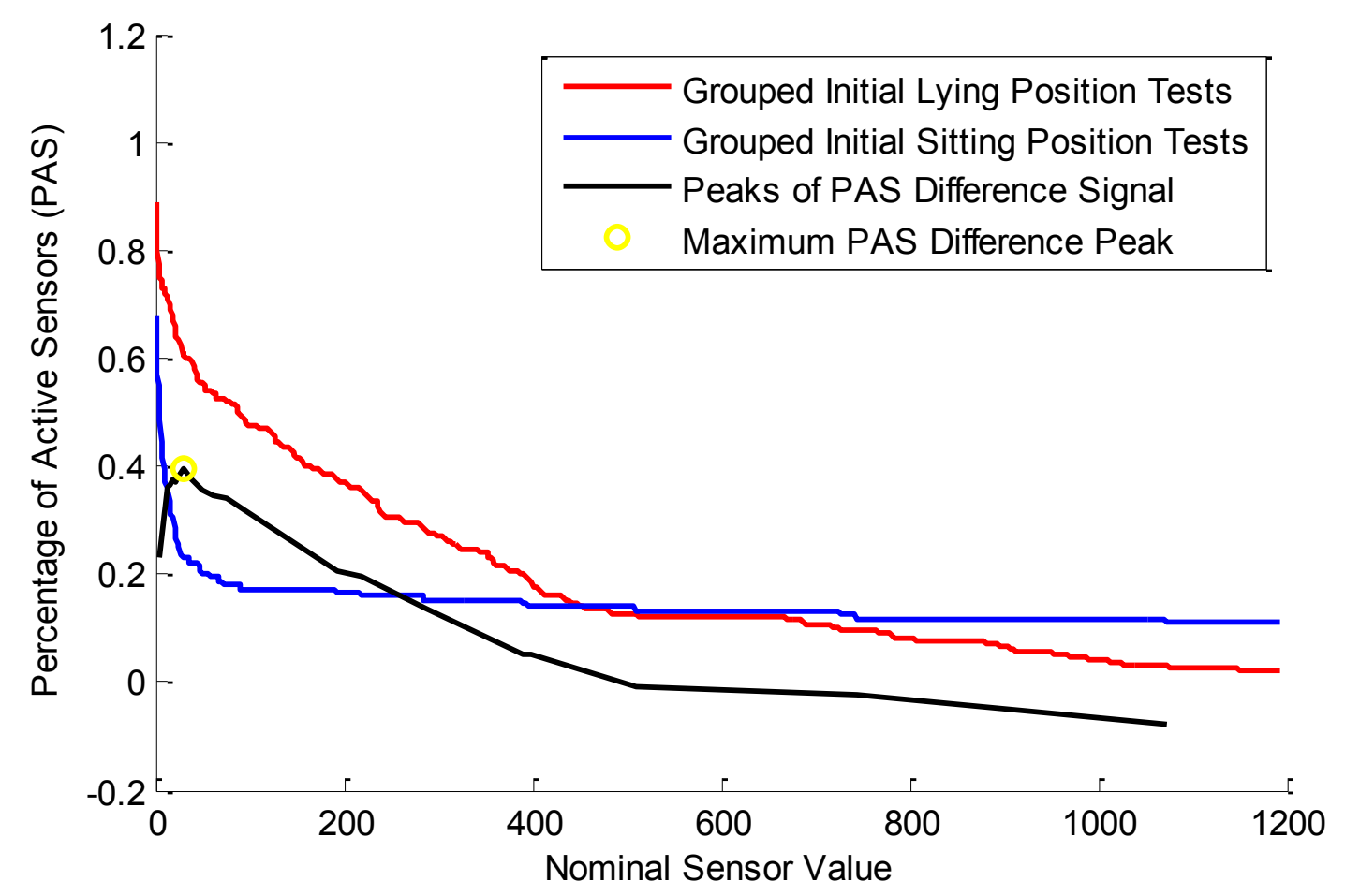

Figure 20: Group average PAS and PAS differences between the two motion groups at varying threshold values.

Once an individualized participant threshold value was determined, the NAS was calculated again as per equations 10 and 11 where $t$ represents points in time, $\{r, c\}$ represents the sensor position in a 9 by 8 matrix and $\{p, s c\}$ stands for the participant and scenario number. The percentage of active sensors was calculated as per Equation 12.

$$
\begin{gathered}
N A S\{p, s c\}(t)=\sum_{r=1}^{9} \sum_{c=1}^{8} A(C I S D\{p, s c\}\{r, c\}(t)) \\
A(C I S D\{p, s c\}\{r, c\}(t))= \begin{cases}1 & \text { if } \operatorname{CISD}\{p, s c\}\{r, c\}(t)>\operatorname{threshold}(p, s c) \\
0 & \text { otherwise }\end{cases} \\
P A S\{p, s c\}(t)=\frac{N A S\{p, s c\}(t)}{72}
\end{gathered}
$$

These individualized thresholds improve the PAS feature, illustrated in Figure 21, where the left image depicts active sensors exceeding a threshold of sensor score 0 and 
the right image depicts active sensors exceeding the individualized threshold for the example participant.
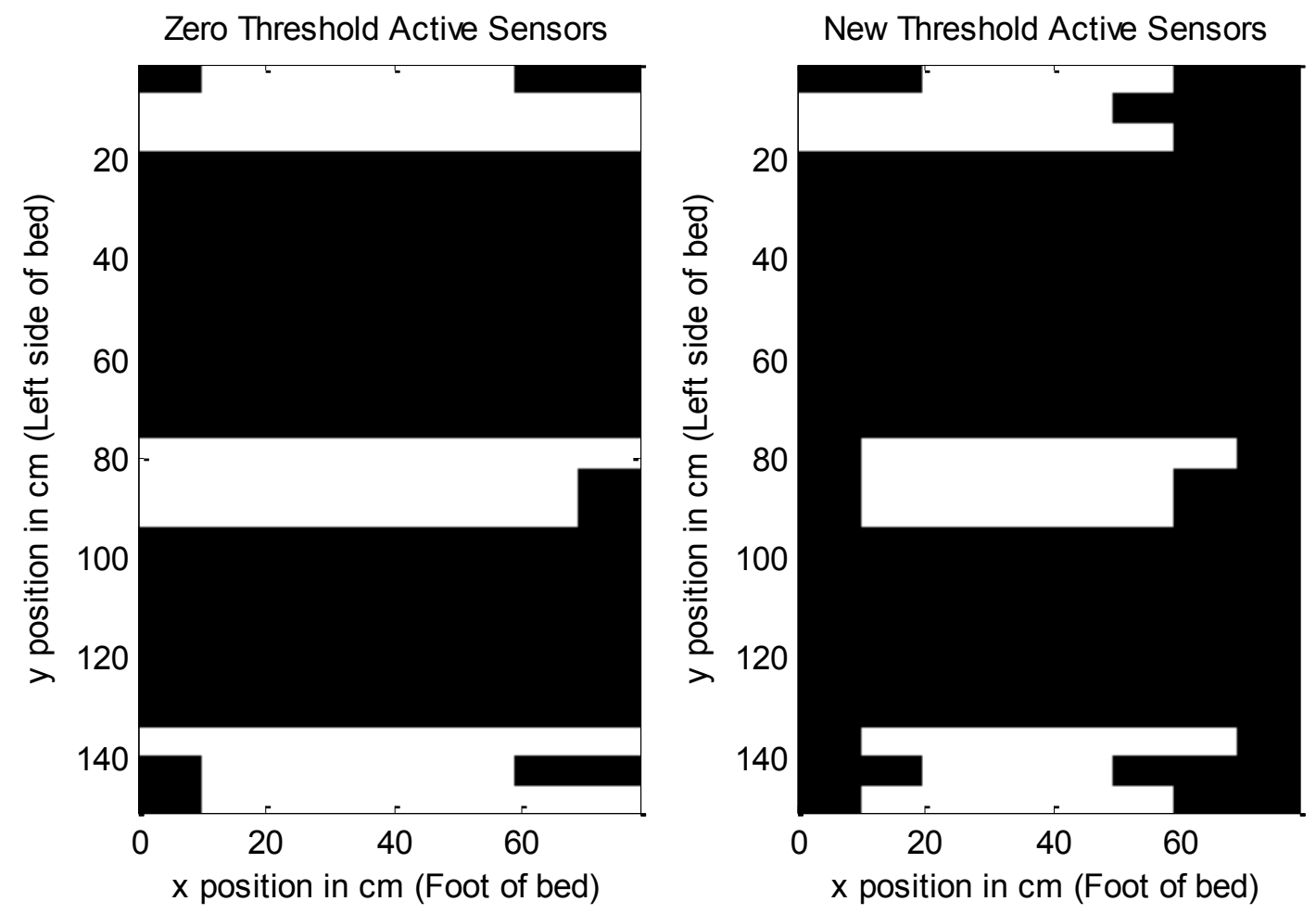

Figure 21: PAS for the example participant in a supine lying posture with a zero and individualized threshold.

\subsubsection{LonCP: Longitudinal Center of Pressure}

The longitudinal center of pressure is a measure of central pressure location underneath a subject on the bed in the $\mathrm{x}$ direction (direction axes are indicated in Figure 22). This feature was used in [59], and is examined in this chapter as a possible feature for emphasizing differences in pressure between a supine lying and sitting position. 


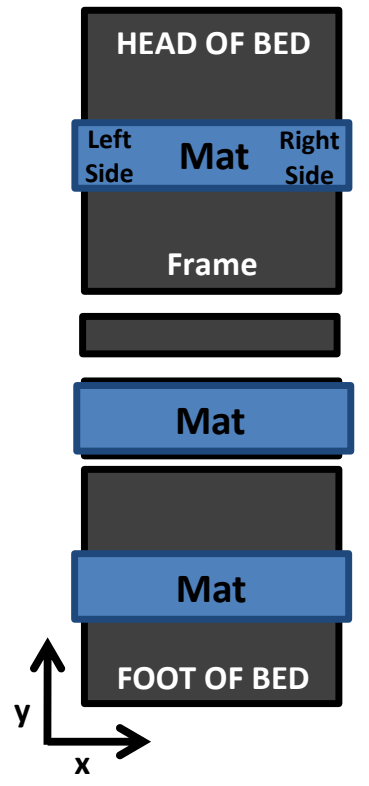

Figure 22: Schematic of pressure mats as they are placed on the bed frame.

This measure is calculated as the center of mass (in the $\mathrm{x}$ direction) would be calculated for a rigid body in physics; the equations for which can be seen in equations 13 and 14. The variable $t$ refers to respective points in time, $x(c)$ refers to the location of the sensor in the $\mathrm{x}$ direction, is measured in centimeters, and is a function of the sensor column location. $\operatorname{CISD}\{p, s c\}\{r, c\}(t)$ refers to calibrated sensor values up to $t=4 \mathrm{sec}$. The variable $T S\{p, s c\}(t)$ is the total sum of sensor values at every point in time, used in the calculation of $\operatorname{Lon} C P\{p, s c\}(t)$.

$$
\begin{gathered}
\operatorname{LonCP}\{p, s c\}(t)=\frac{1}{T S\{p, s c\}(t)} \sum_{r=1}^{9} \sum_{c=1}^{8} x(c) * C I S D\{p, s c\}\{r, c\}(t) \\
T S\{p, s c\}(t)=\sum_{r=1}^{9} \sum_{c=1}^{8} \operatorname{CISD}\{p, s c\}\{r, c\}(t)
\end{gathered}
$$




\subsubsection{LatCP: Latitudinal Center of Pressure}

The latitudinal center of pressure, is a measure of central pressure underneath a subject on the bed in the y direction (direction axes are indicated in Figure 22. This measure is again calculated as the center of mass (in the y direction) would be calculated for a rigid body in physics. The statistical algorithms used can be seen in equations 15 and 14, where $t$ refers to the point in time, $y(r)$ refers to the location of the sensor in the $\mathrm{y}$ direction, and $\operatorname{CISD}\{p, s c\}\{r, c\}(t)$ refers to respective sensor values up to $t=4 s e c$. The variable $\operatorname{TS}\{p, s c\}(t)$ is described in Equation 14, and used only for the calculation of $\operatorname{LatCP}\{p, s c\}(t)$ and $\operatorname{LonCP}\{p, s c\}(t)$.

$$
\operatorname{LatCP}\{p, s c\}(t)=\frac{1}{T S\{p, s c\}(t)} \sum_{r=1}^{9} \sum_{c=1}^{8} y(r) * \operatorname{CISD}\{p, s c\}\{r, c\}(t)
$$

\subsubsection{LonV: Longitudinal Variance}

The variable NAS was further examined by exploring the statistical spread of active sensors in the $\mathrm{x}$ direction, otherwise known as the longitudinal variance. Theoretically, a subject that is lying down should cover a larger area than a subject that is sitting and therefore the spread, or variance, should be higher. The addition of a feature that emphasizes the difference between sitting and standing is an ideal feature for a classifier in this subsystem. The longitudinal variance was calculated as in Equation 16 where $A(C I S D\{p, s c\}\{r, c\}(t))$ is a step function detailed in Equation 11 that allocates a value of 0 if the signal $\operatorname{CISD}\{p, s c\}\{r, c\}(t)$ surpasses the participant threshold determined in 
section 5.2.1. The variable $x(c)$ is the sensor location in centimeters and $\bar{x}\{p, s c\}(t)$ is calculated as in Equation 17.

$$
\begin{gathered}
\operatorname{LonV}\{p, s c\}(t)=\frac{1}{N A S\{p, s c\}(t)} \sum_{r=1}^{9} \sum_{c=1}^{8} A(C I S D\{p, s c\}\{r, c\}(t)) *(x(c)-\bar{x}\{p, s c\}(t))^{2} \\
\bar{x}\{p, s c\}(t)=\frac{1}{N A S\{p, s c\}(t)} \sum_{r=1}^{9} \sum_{c=1}^{8} A(C I S D\{p, s c\}\{r, c\}(t)) * x(c)
\end{gathered}
$$

\subsubsection{LatV: Latitudinal Variance}

The statistical spread of active sensors was also examined in the y direction and referred to here as the latitudinal variance. The latitudinal variance was calculated as in Equation 18, where $A(C I S D\{p, s c\}\{r, c\}(t))$ is the step function described in Equation $11, y(r)$ is again the sensor location and $\bar{y}\{r, c\}(t)$ is calculated as in Equation 19.

$$
\begin{gathered}
\operatorname{LatV}\{p, s c\}(t)=\frac{1}{N A S\{p, s c\}(t)} \sum_{r=1}^{9} \sum_{c=1}^{8} A(C I S D\{p, s c\}\{r, c\}(t)) *(y(r)-\bar{y}\{p, s c\}(t))^{2} \\
\bar{y}\{p, s c\}(t)=\frac{1}{N A S\{p, s c\}(t)} \sum_{r=1}^{9} \sum_{c=1}^{8} A(C I S D\{p, s c\}\{r, c\}(t)) * y(r)
\end{gathered}
$$

\subsubsection{CLR: Central Left Ratio}

This feature examines the proportion of total pressure residing in the left section of the middle mat. This portion was chosen as it was most often the area occupied by subjects in a sitting position. The calculation for this feature consists of summing the values of the 9 sensors highlighted in yellow in Figure 23, summing the values of the sensors highlighted in blue, then dividing the former sum by the latter. The equation describing this 
calculation can be seen in (20), where CLRsumyellow $\{p, s c\}(t)$ is the sum of sensor values highlighted in yellow, CLRsumblue $\{p, s c\}(t)$ is the sum of sensor values highlighted in blue and the resulting signal is a ratio of pressure of the yellow area to the blue area over time.

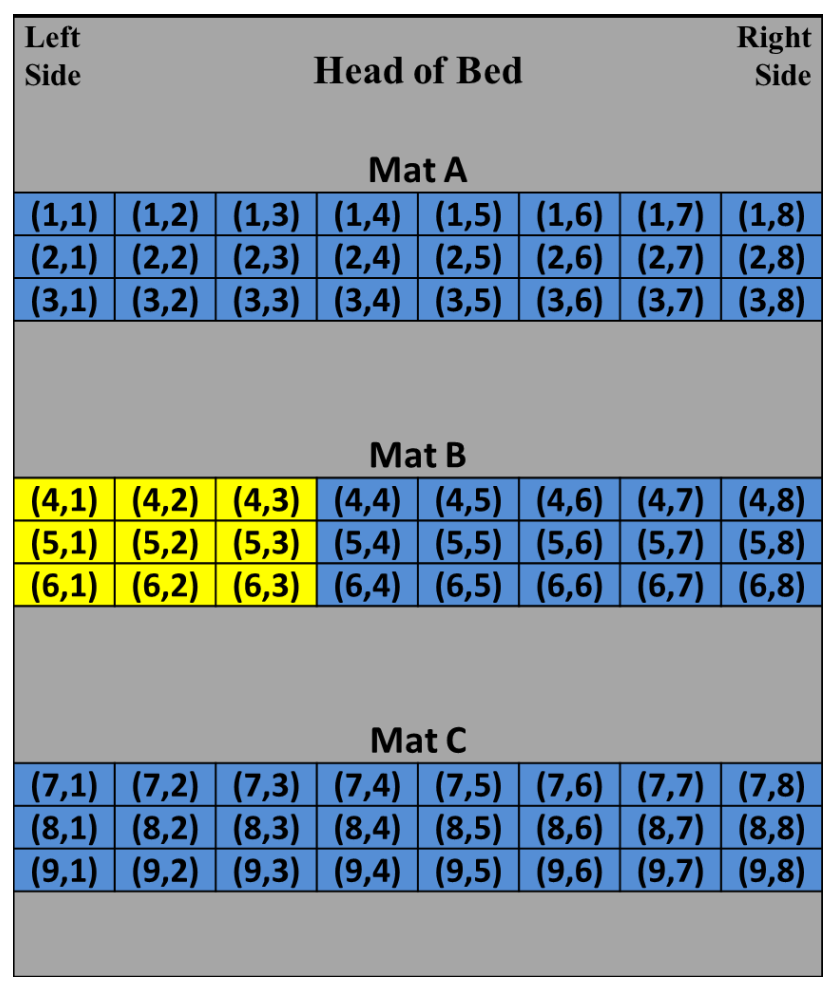

Figure 23: Schematic of the pressure mats with a highlighted central left area.

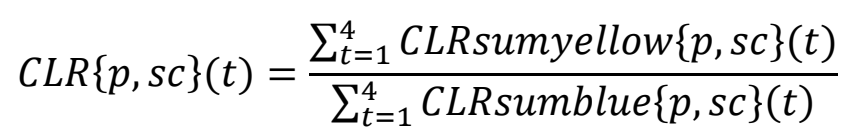

\subsubsection{QLR: Quarter Left Ratio}

The quarter left ratio feature examines the left quarter of all mats as volunteers were instructed to sit on the left edge of the bed for Scenarios 4 and 5. This feature looks at the entire left side of the bed so as to eliminate the assumption that the subject would sit in 
the center on the left side of the bed. This ratio was calculated similarly to the central left ratio. Figure 24 depicts the area of interest, highlighted in yellow. The sensor values associated with the sensors in the yellow highlighted area were summed, the sensor values associated with the sensors in the blue highlighted area were summed and the former sum was divided by the latter. The equation describing this calculation can be seen as Equation 21, where QLRsumyellow $\{p, s c\}(t)$ is the sum of sensor values highlighted in yellow, QLRsumblue $\{p, s c\}(t)$ is the sum of sensor values highlighted in blue and the resulting signal is a ratio of pressure of the yellow area to the blue area over time.

\begin{tabular}{|c|c|c|c|c|c|c|c|}
\hline $\begin{array}{l}\text { Left } \\
\text { Side }\end{array}$ & \multicolumn{6}{|c|}{ Head of Bed } & $\begin{array}{l}\text { Right } \\
\text { Side }\end{array}$ \\
\hline$(1,1)$ & $(1,2)$ & $(1,3)$ & $(1,4)$ & $(1,5)$ & $(1,6)$ & $(1,7)$ & $(1,8)$ \\
\hline$(2,1)$ & $(2,2)$ & $(2,3)$ & $(2,4)$ & $(2,5)$ & $(2,6)$ & $(2,7)$ & $(2,8)$ \\
\hline$(3,1)$ & $(3,2)$ & $(3,3)$ & $(3,4)$ & $(3,5)$ & $(3,6)$ & $(3,7)$ & $(3,8)$ \\
\hline \multicolumn{8}{|c|}{ Mat B } \\
\hline$(4,1)$ & $(4,2)$ & $(4,3)$ & $(4,4)$ & $(4,5)$ & $(4,6)$ & $(4,7)$ & $(4,8)$ \\
\hline$(5,1)$ & $(5,2)$ & $(5,3)$ & $(5,4)$ & $(5,5)$ & $(5,6)$ & $(5,7)$ & $(5,8)$ \\
\hline$(6,1)$ & $(6,2)$ & $(6,3)$ & $(6,4)$ & $(6,5)$ & $(6,6)$ & $(6,7)$ & $(6,8)$ \\
\hline \multicolumn{8}{|c|}{ Mat C } \\
\hline$(7,1)$ & $(7,2)$ & $(7,3)$ & $(7,4)$ & $(7,5)$ & $(7,6)$ & $(7,7)$ & $(7,8)$ \\
\hline$(8,1)$ & $(8,2)$ & $(8,3)$ & $(8,4)$ & $(8,5)$ & $(8,6)$ & $(8,7)$ & $(8,8)$ \\
\hline$(9,1)$ & $(9,2)$ & $(9,3)$ & $(9,4)$ & $(9,5)$ & $(9,6)$ & $(9,7)$ & $(9,8)$ \\
\hline
\end{tabular}

Figure 24: Schematic of the pressure mats with a highlighted quarter left area.

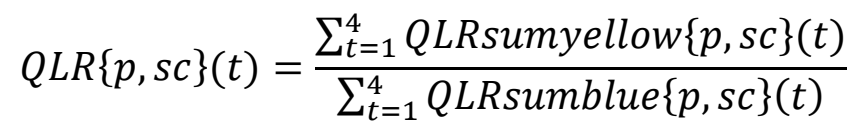




\subsection{Feature Evaluation and Selection}

Once the features were calculated, each feature was evaluated for its ability to distinguish between the two positions of interest in this chapter. This was done first on an individual basis, with the intent of developing an effective classifier for this stage of the system. In [59] three different types of classifiers were examined in order to distinguish between six different lying and sitting orientations: support vector machine (SVM), nearest neighbour $(\mathrm{NN})$ and k-nearest neighbour $(\mathrm{kNN})$. The SVM classifier, with varying combinations of features, was the best performer for static activity recognition and for lying posture recognition with both subject-dependent and subject-independent data. This classifier was built using a Gaussian Radial Based Function (RBF) kernel, for which the hyperparameters were selected using leave-one-out cross validation for subject-independent data. Based on the similarities between the classifier used in [59] and the classifier needed in this subsystem, an SVM classifier with a linear kernel and leave-one-subjectout cross validation was used. A learning system rule of thumb is to look for the simplest solution that yields good results [64]. For this reason, a linear discriminant classifier with leave-one-subject-out cross validation was also used and the results were compared. Each feature was examined separately and the best performing features were further examined by adding Gaussian noise and re-evaluating feature performance. A final model was

determined by using the best performing features with the best performing classifier and all of the patient and scenario data. 


\subsection{Results and Discussion}

An iterative process was used to train both an SVM (with a linear kernel) and a linear discriminant classifier. Data from all scenarios enacted by all except one participant was used to train both classifiers. This meant that one full set of five enacted scenarios was excluded from the training data. These five scenarios from one participant were used to test each classifier, and the accuracy of each classifier was recorded. This was done iteratively 30 times, so as to test the classifiers with each participant. The average accuracies from this iterative process were found can be seen in Table 6 .

Table 6: Features examined and the corresponding classifier accuracies

\begin{tabular}{|c|c|c|}
\hline Feature & SVM Accuracy & Linear Accuracy \\
\hline \hline PAS & 1.00 & 1.00 \\
\hline LonCP & 0.98 & 0.98 \\
\hline LatCP & 0.98 & 0.91 \\
\hline LonV & 0.67 & 0.67 \\
\hline LatV & 1.00 & 1.00 \\
\hline CMR & 0.72 & 0.77 \\
\hline QLR & 0.99 & 0.99 \\
\hline
\end{tabular}

The results from the two classifiers are very similar, with PAS and LatV displaying good predictive qualities. If features have good predictive qualities, any of many classification methods should perform well [64]. Because a simpler solution with good results tends to generalize better than a complicated one [64], the linear discriminant classifier is an advantageous choice.

Most features were close to total accuracy; the features PAS and LatV, highlighted in yellow, resulted in total accuracy. The feature QLR is also highlighted in yellow and further examined as it was the next closest feature to total accuracy. These 
feature data points are plotted one-dimensionally in Figure 25, indicating the spread of values, the difference in groups and the linear classifier determined threshold. In order to determine which, if any of these features should be excluded from a final classifier model, the feature signals were first normalized to best compare feature performance. White Gaussian noise was then added at a Signal to Noise Ratio (SNR) of 10, and then 5 to test the sensitivity of the features. These new signals were used with a linear discriminant classifier and again evaluated for accuracy. These results can be seen in Table 7.
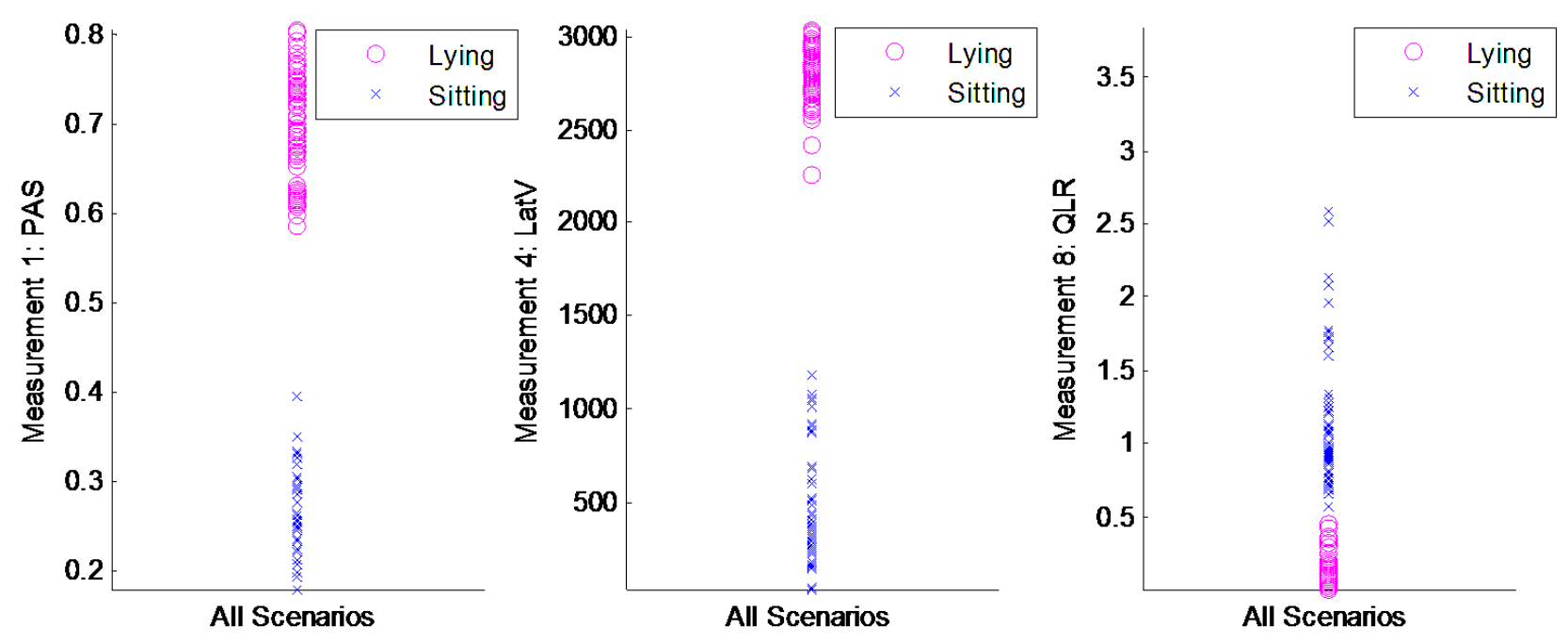

Figure 25: All data from the features with total accuracy

Table 7: Features and resulting accuracies after the addition of noise.

\begin{tabular}{|c|c|c|}
\cline { 2 - 3 } \multicolumn{1}{c|}{} & \multicolumn{2}{c|}{ Linear Classifier Accuracy } \\
\hline \hline Feature & SNR=10 & SNR $=\mathbf{5}$ \\
\hline \hline PAS & 0.90 & 0.80 \\
\hline LatV & 0.91 & 0.75 \\
\hline QLR & 0.63 & 0.63 \\
\hline
\end{tabular}


The best performing feature was LatV, followed by PAS and then QLR. The top two features are less dependent on subject orientation than QLR; if a subject was sit on the right edge of the bed, the feature QLR would be useless. The feature QLR was therefore excluded, and a final liner classifier model was created using all patient data and the features PAS and LatV. This final model resulted in total accuracy, with a linear threshold depicted in Figure 26 and described in Equation 22.

$$
y=\left(-3.52 * 10^{-4}\right) x+1.04
$$

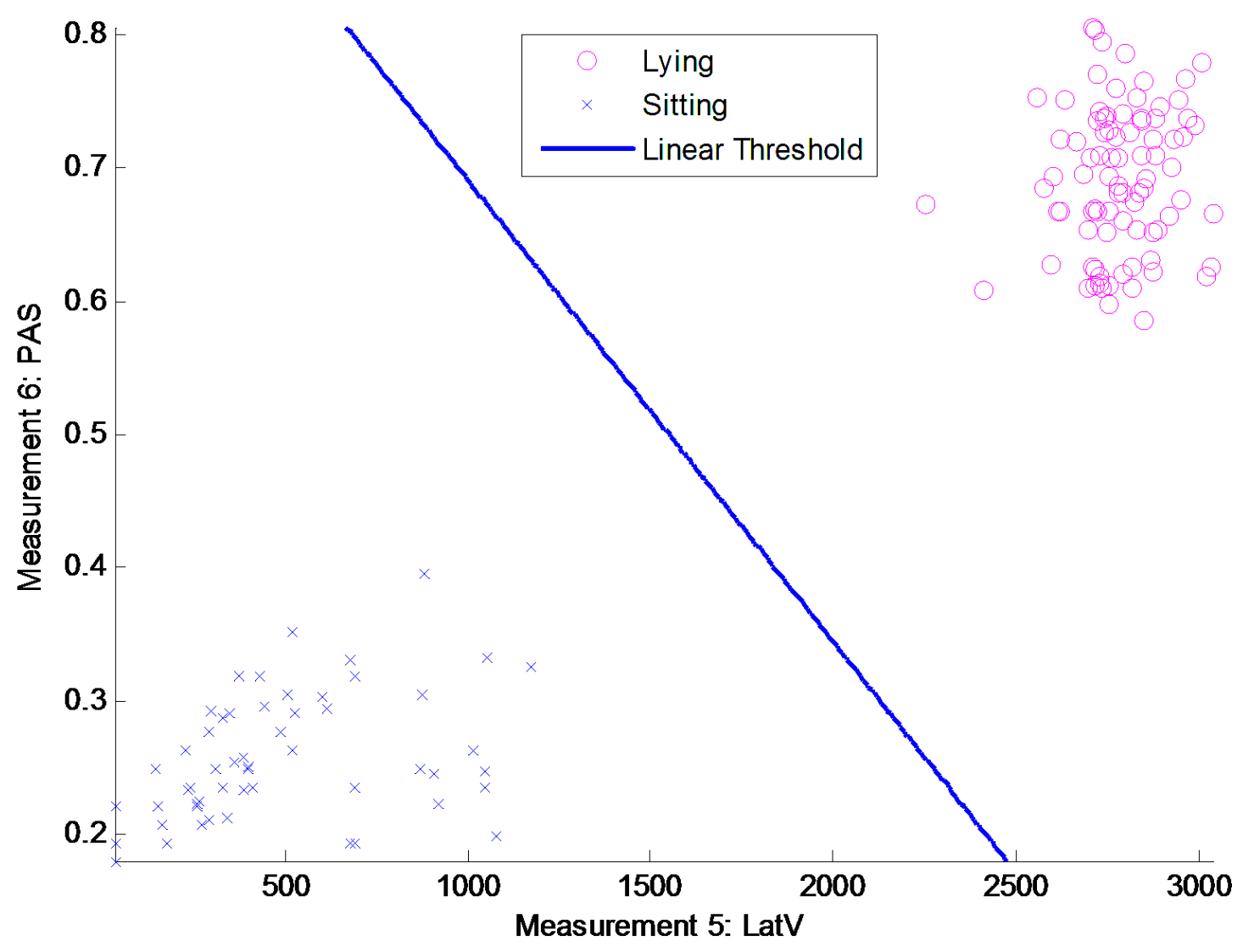

Figure 26: Final classified data and classifier threshold

This stage of the system was therefore able to fully distinguish sitting positions from lying positions. An unidentified data set analyzed by this stage of the system would 
be passed through one of two secondary classification stages depending on the classification result of DIP. These secondary stages are detailed in the following two chapters. 


\section{Chapter 6}

\section{Distinction Between Lying Scenarios.}

This chapter details a secondary stage of the algorithmic system developed for this thesis, called the Distinction Between Lying Scenarios, or DBLS.

\subsection{Introduction}

Distinction Between Lying Scenarios (DBLS), depicted in Figure 27, receives data from DIP, the first stage of the system determining the initial position of the participant. If the initial position is determined to be supine and lying, data is passed to DBLS, which aims to determine which of three enactments the data represents. The three enactments to be distinguished between are Scenario 1: Needs positioning in bed, Scenario 2: Can position self in bed and Scenario 3: Lying to sitting independently.

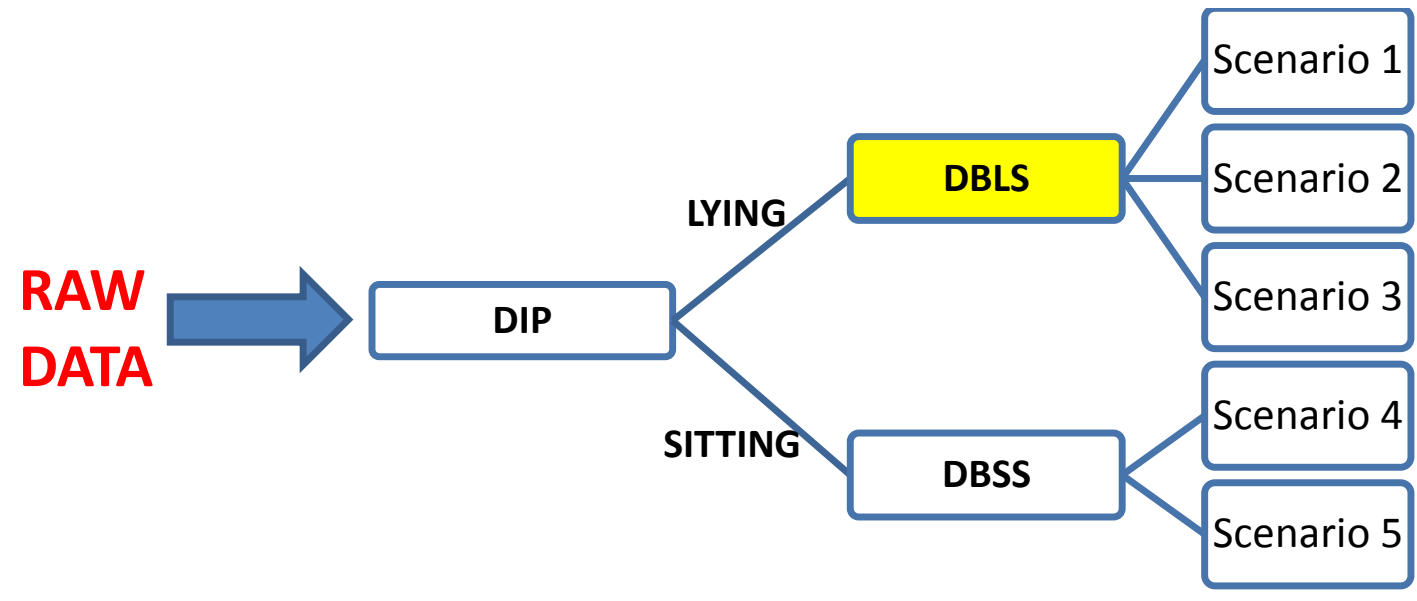

Figure 27: System tree describing the role of DBLS respective to the entire system. 
The system stage detailed in this chapter aims to distinguish between the three scenarios in question through the identification and monitoring of pressure and pressure relief in areas of interest. Different areas are of interest for Scenario 3 than scenarios 1 and 2. For this reason, DBLS is broken into two stages as seen in in Figure 28. The first stage monitors pressure changes in the mat the head of the bed to determine if data represents Scenario 3. If not, the data is passed to the second stage, which uses region based identification and monitoring of pressure points to distinguish between scenarios 1 and 2. Algorithmic methods, results and discussion are examined in the following sections. A summary of variable acronyms used in the following sections is in Table 8 .

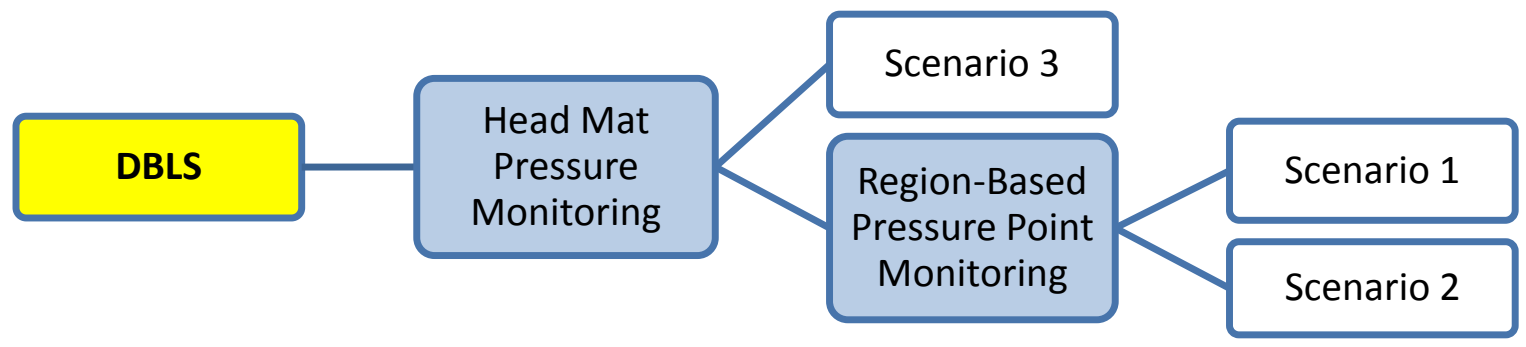

Figure 28: System tree describing the architecture of Subsystem 2.

Table 8: Summary of variable acronyms for DBLS

\begin{tabular}{|c|c|c|c|}
\hline Variable & $\begin{array}{c}\text { Variable } \\
\text { Acronym }\end{array}$ & $\begin{array}{c}\text { Chapter } \\
\text { Section }\end{array}$ & $\begin{array}{c}\text { Equation } \\
\text { Numbers }\end{array}$ \\
\hline \hline Sum of Sensors in Mat A & SMA & 6.2 & 23 \\
\hline Baseline of Mat A & BMA & 6.2 & 24 \\
\hline Percentage Increase of Mat A & PIMA & 6.2 & 25 \\
\hline Percentage Decrease of Mat A & PDMA & 6.2 & 26 \\
\hline Baseline Pressure for Individual sensors & BPI & 6.3 & 27 \\
\hline Pressure Increase for Individual sensors & PII & 6.3 & 28 \\
\hline Pressure Decrease for Individual sensors & PDI & 6.3 & 29 \\
\hline
\end{tabular}




\subsection{Head Mat Pressure Monitoring}

This ratio-based weight distribution algorithm determined whether not a volunteer was enacting Scenario 3, a HABAM Mobility score of 7: 'Lying-sitting independently'. This scenario should theoretically be characterized by a slow relief of pressure of the top mat and an increase in pressure exerted on the mats at the middle and foot of the bed, as the participant moves from a lying position to a sitting position. This stage of DBLS therefore assumes the characteristic pressure pattern and looks for prolonged pressure relief of the top mat (i.e. not a single frame of relief representative of $0.1 \mathrm{~s}$ ).

For each volunteer, enactment and point in time, data from sensors in Mat A (highlighted in yellow) were summed as depicted in Figure 29. Equivalently, this was the sum of the first three calibrated row sums $(r 1, r 2$ and $r 3)$, detailed in Chapter 3.5.1. This calculation is described in Equation 23, where $S M A\{p, s c\}(t)$ is the summed data from Mat A.

$$
S M A\{p, s c\}(t)=r 1\{p, s c\}(t)+r 2\{p, s c\}(t)+r 3\{p, s c\}(t)
$$




\begin{tabular}{|c|c|c|c|c|c|c|c|}
\hline $\begin{array}{l}\text { Left } \\
\text { Side }\end{array}$ & & & Head & of Bed & & & $\begin{array}{r}\text { Right } \\
\text { Side }\end{array}$ \\
\hline & & & & t A & & & \\
\hline$(1,1)$ & $(1,2)$ & $(1,3)$ & $(1,4)$ & $(1,5)$ & $(1,6)$ & $(1,7)$ & $(1,8)$ \\
\hline$(2,1)$ & $(2,2)$ & $(2,3)$ & $(2,4)$ & $(2,5)$ & $(2,6)$ & $(2,7)$ & $(2,8)$ \\
\hline$(3,1)$ & $(3,2)$ & $(3,3)$ & $(3,4)$ & $(3,5)$ & $(3,6)$ & $(3,7)$ & $(3,8)$ \\
\hline & & & & t B & & & \\
\hline$(4,1)$ & $(4,2)$ & $(4,3)$ & $(4,4)$ & $(4,5)$ & $(4,6)$ & $(4,7)$ & $(4,8)$ \\
\hline$(5,1)$ & $(5,2)$ & $(5,3)$ & $(5,4)$ & $(5,5)$ & $(5,6)$ & $(5,7)$ & $(5,8)$ \\
\hline$(6,1)$ & $(6,2)$ & $(6,3)$ & $(6,4)$ & $(6,5)$ & $(6,6)$ & $(6,7)$ & $(6,8)$ \\
\hline & & & & t C & & & \\
\hline$(7,1)$ & $(7,2)$ & $(7,3)$ & $(7,4)$ & $(7,5)$ & $(7,6)$ & $(7,7)$ & $(7,8)$ \\
\hline$(8,1)$ & $(8,2)$ & $(8,3)$ & $(8,4)$ & $(8,5)$ & $(8,6)$ & $(8,7)$ & $(8,8)$ \\
\hline$(9,1)$ & $(9,2)$ & $(9,3)$ & $(9,4)$ & $(9,5)$ & $(9,6)$ & $(9,7)$ & $(9,8)$ \\
\hline
\end{tabular}

Figure 29: Schematic of the mats for head mat pressure monitoring.

The change in pressure exerted on the mats was found by first determining a baseline pressure reading for the subject on Mat A. The baseline value was the average of the first 4 seconds (equivalent to 40 data frames) of each enactment by each volunteer, as this was the time during which the participant was reliably lying still on the hospital bed. This calculation is seen in Equation 24, where $B M A(p, s c)$ is the average baseline reading for Mat $\mathrm{A}$.

$$
B M A(p, s c)=\frac{1}{40} \sum_{t=1}^{4 s e c} S M A\{p, s c\}(t)
$$

In order to best eliminate the dependency of results on participant height and weight, the percentage change from baseline pressure in Mat A was used instead of absolute sensor values. At every point in time the percentage change from baseline for 
Mat A was calculated. This change was considered as either a percentage increase or a percentage decrease, described in equations 25 and 26 below, and calculated for each enactment. The variables $P I M A\{p, s c\}(t)$ and $P D M A\{p, s c\}(t)$ are percentage increase and percentage decrease respectively. As with related studies, percentage variables were then filtered via a moving average filter with a window of $\mathrm{W}=5$ to decrease noise [61].

$$
\begin{aligned}
P I M A\{p, s c\}(t) & =\frac{\|S M A\{p, s c\}(t)-B M A(p, s c)\|}{B M A(p, s c)} \\
P D M A\{p, s c\}(t) & =\frac{-\|S M A\{p, s c\}(t)-B M A(p, s c)\|}{B M A(p, s c)}
\end{aligned}
$$

A percentage decrease of -1.0 means that the original pressure has been totally relieved. Due to the weight distributing effects of the mattress, pressure relief isn't always represented by a percentage decrease of -1.0. This is particularly true for Scenario 3, which was enacted in many different ways not originally anticipated. Participants were seen to: use their feet to move their sacrum region back towards their hands while sitting up, to sit up without the use of their arms, to roll to one side and then use their arms to lift their bodies, to use their arms to sit up halfway and then stop, amongst other methods. For this reason, and because the top mat represents a significant area experiencing changes in pressure exerted by the hospital mattress as a result of participant movement, this stage of the system looks for at least an $80 \%$ decrease in pressure exerted on the top mat. A condition was therefore applied to unidentified data received by DBLS; if $P D M A\{p, s c\}(t)$ drops to -0.8 or below, the enactment was considered to be of Scenario 3, otherwise the unidentified data was passed on to the next stage of DBLS. 


\subsection{Region Based Pressure Monitoring}

If pressure monitoring of Mat A did not result in identification of the enactment data, the data set was passed to the second stage, region-based pressure monitoring. This stage consists of algorithms that determine whether a volunteer enacted Scenario 1, a HABAM Mobility score of 0: 'Needs Positioning in Bed' or Scenario 2, a HABAM mobility score of 4: 'Positions Self in Bed'. This is done using region-based monitoring of points of high pressure and identification of specific pressure patterns characteristic of respective scenarios.

The data were first separated in six groups by separating each mat into left and right sides. This was done to identify regions in which different pressure points resided, aiding in the detection of these points of high pressure. The regions indicated areas of interest underneath a supine lying volunteer; underneath the scapula (and/or head depending on volunteer height and orientation), underneath the hips and underneath the heels. Each group includes 12 sensors and can be seen in the schematic in Figure 30, where the groups are named Head Left (HL), Head Right (HR), Mid Left (ML), Mid Right (MR), Foot Left (FL) and Foot Right (FR). No sums or ratios were calculated; the sensors were just considered part of respective groups. 


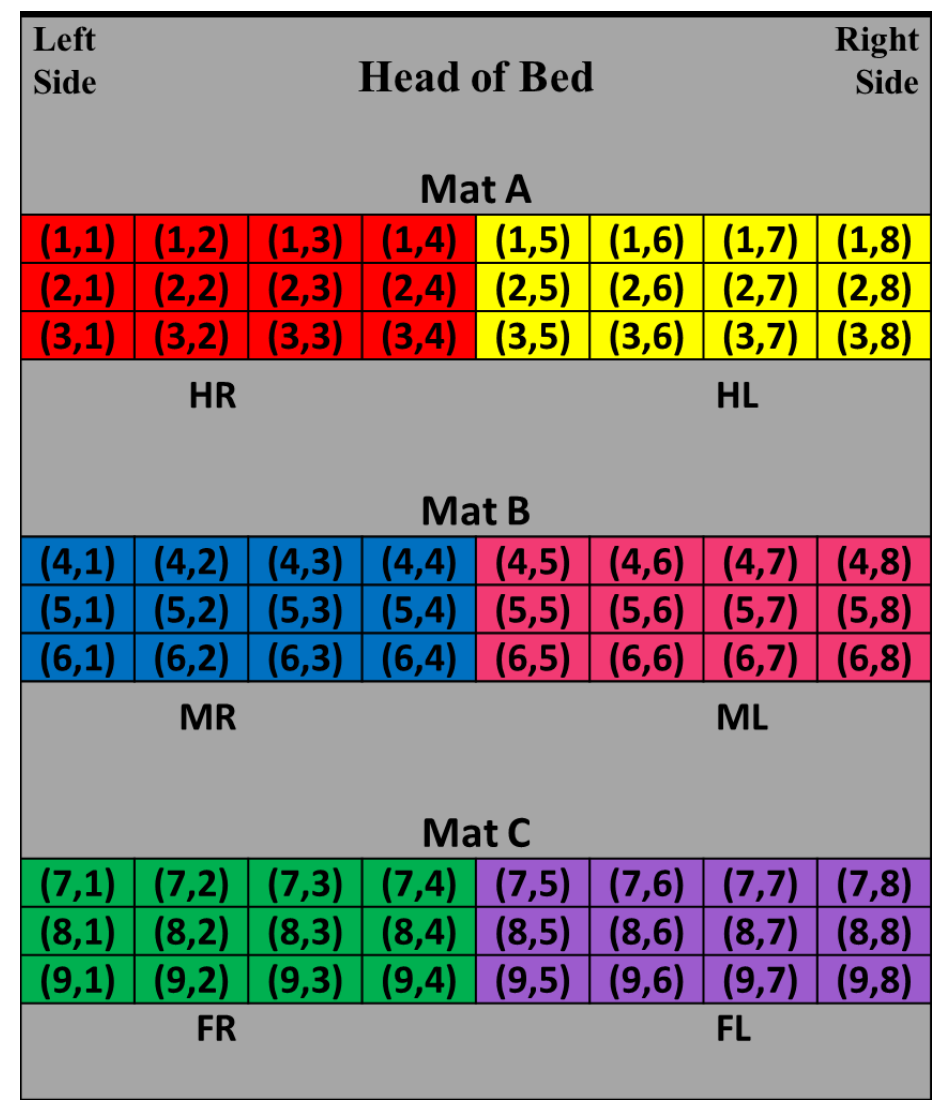

Figure 30: Schematic of the groupings for region based pressure monitoring.

As with the previous section, percentage pressure change was calculated in order to identify pressure relief. Unlike the previous section, this stage of DBLS examines pressure changes on an individual sensor basis. For each sensor in each group, an average of the first 4 seconds of each enactment by each volunteer was calculated. This gave a baseline pressure reading for a subject on the mats on an individual sensor basis $(B P I\{p, s c\}(r, c))$, as described in Equation 27.

$$
B P I\{p, s c\}(r, c)=\frac{1}{40} \sum_{t=1}^{4 s e c} C I S D\{p, s c\}\{r, c\}(t)
$$


The observed pressure pattern seen for subjects lying supine and rolling to one side, is an increase in pressure on the side to which the subject is rolling, and a relief of pressure on the opposite side of the bed. For this reason, and for the purposes of minimizing dependency on volunteer height and weight, percentage change over time is used to detect this pattern of increase and decrease. For every sensor, the percentage change over time from baseline was calculated. This change was considered as either a percentage increase or a percentage decrease as indicated in equations 28 and 29 below, which were calculated for each enactment. The variable $\operatorname{CISD}\{p, s c\}\{r, c\}(t)$ is the calibrated individual sensor data described in Section 3.5.2, BPI $\{p, s c\}(r, c)$ is the baseline pressure reading, $\operatorname{PII}\{p, s c\}\{r, c\}(t)$ is percentage increase, and $\operatorname{PDI}\{p, s c\}\{r, c\}(t)$ is percentage decrease for individual sensors. These percentage variables were then filtered via a moving average filter with a window of $\mathrm{W}=5$ as in the previous section.

$$
\begin{gathered}
P I I\{p, s c\}\{r, c\}(t)=\frac{\|C I S D\{p, s c\}\{r, c\}(t)-B P I\{p, s c\}(r, c)\|}{B P I\{p, s c\}(r, c)} \\
P D I\{p, s c\}\{r, c\}(t)=\frac{-\|C I S D\{p, s c\}\{r, c\}(t)-B P I\{p, s c\}(r, c)\|}{B P I\{p, s c\}(r, c)}
\end{gathered}
$$

Again, a percentage decrease of -1.0 means that all the original pressure exerted on an individual sensor has been relieved; however -0.95 is used in this thesis to allow for a small amount of error (0.05) that accounts for the weight distributing effects of the mattress. This stage of the system therefore aimed to identify instances during which at least two sensors had simultaneously dropped below a percentage change of -0.95 . Individual sensors output varying values and only the points of high pressure are of interest; for example a sensor with an original nominal score of 100 may drop to 5, while 
another sensor may drop from a score of 1000 to 50 . The former sensor score drop is likely to be a result of the hospital mattress dispersing applied pressure, while the latter sensor score drop is likely the result of applied, then relieved pressure. A threshold value was needed to determine what baseline sensor scores were considered significant, and what scores were considered insignificant. This threshold value was found using sensor scores and a linear discriminant classifier.

An individual participant threshold was determined to account for varying heights, weights and slightly different orientations. Two groups of data were established from baseline data $(B P I\{p, s c\}(r, c))$; one group of baseline sensor scores likely a result of the distributing effects of the hospital mattress (outside), and another group of significant values likely to be directly underneath a supine, lying participant (inside). The sensors of interest are seen in Figure 31 highlighted in yellow and red, respectively.

\begin{tabular}{|c|c|c|c|c|c|c|c|}
\hline $\begin{array}{l}\text { Left } \\
\text { Side }\end{array}$ & & & Head & f Bed & & & $\begin{array}{r}\text { Right } \\
\text { Side }\end{array}$ \\
\hline \multicolumn{8}{|c|}{$\frac{2}{\text { Mat A }}$} \\
\hline$(2,1)$ & $(2,2)$ & $(2,3)$ & $(2,4)$ & $(2,5)$ & $(2,6)$ & $(2,7)$ & $(2,8)$ \\
\hline$(3,1)$ & $(3,2)$ & $(3,3)$ & $(3,4)$ & $(3,5)$ & $(3,6)$ & $(3,7)$ & $(3,8)$ \\
\hline \multicolumn{8}{|c|}{ Mat B } \\
\hline$(4,1)$ & $(4,2)$ & $(4,3)$ & $(4,4)$ & $(4,5)$ & $(4,6)$ & $(4,7)$ & $(4,8)$ \\
\hline$(5,1)$ & $(5,2)$ & $(5,3)$ & $(5,4)$ & $(5,5)$ & $(5,6)$ & $(5,7)$ & $(5,8)$ \\
\hline$(6,1)$ & $(6,2)$ & $(6,3)$ & $(6,4)$ & $(6,5)$ & $(6,6)$ & $(6,7)$ & $(6,8)$ \\
\hline \multicolumn{8}{|c|}{ Mat C } \\
\hline$(7,1)$ & $(7,2)$ & $(7,3)$ & $(7,4)$ & $(7,5)$ & $(7,6)$ & $(7,7)$ & $(7,8)$ \\
\hline$(8,1)$ & $(8,2)$ & $(8,3)$ & $(8,4)$ & $(8,5)$ & $(8,6)$ & $(8,7)$ & $(8,8)$ \\
\hline$(9,1)$ & $(9,2)$ & $(9,3)$ & $(9,4)$ & $(9,5)$ & $(9,6)$ & $(9,7)$ & $(9,8)$ \\
\hline
\end{tabular}

Figure 31: Schematic of the significant verses insignificant sensor scores. 
A linear discriminant classifier was used to determine an optimal threshold between significant sensor scores and insignificant sensor scores. An example of this can be seen in Figure 32, where the blue data points represent sensors of significant value, the pink data points represent sensors of insignificant value and the blue line is the threshold determined by the classifier. The threshold depicted did not result in total accuracy, as an assumption was made as to where significant pressure resided, but is a reasonable estimation of an individualized threshold for this stage of the system.

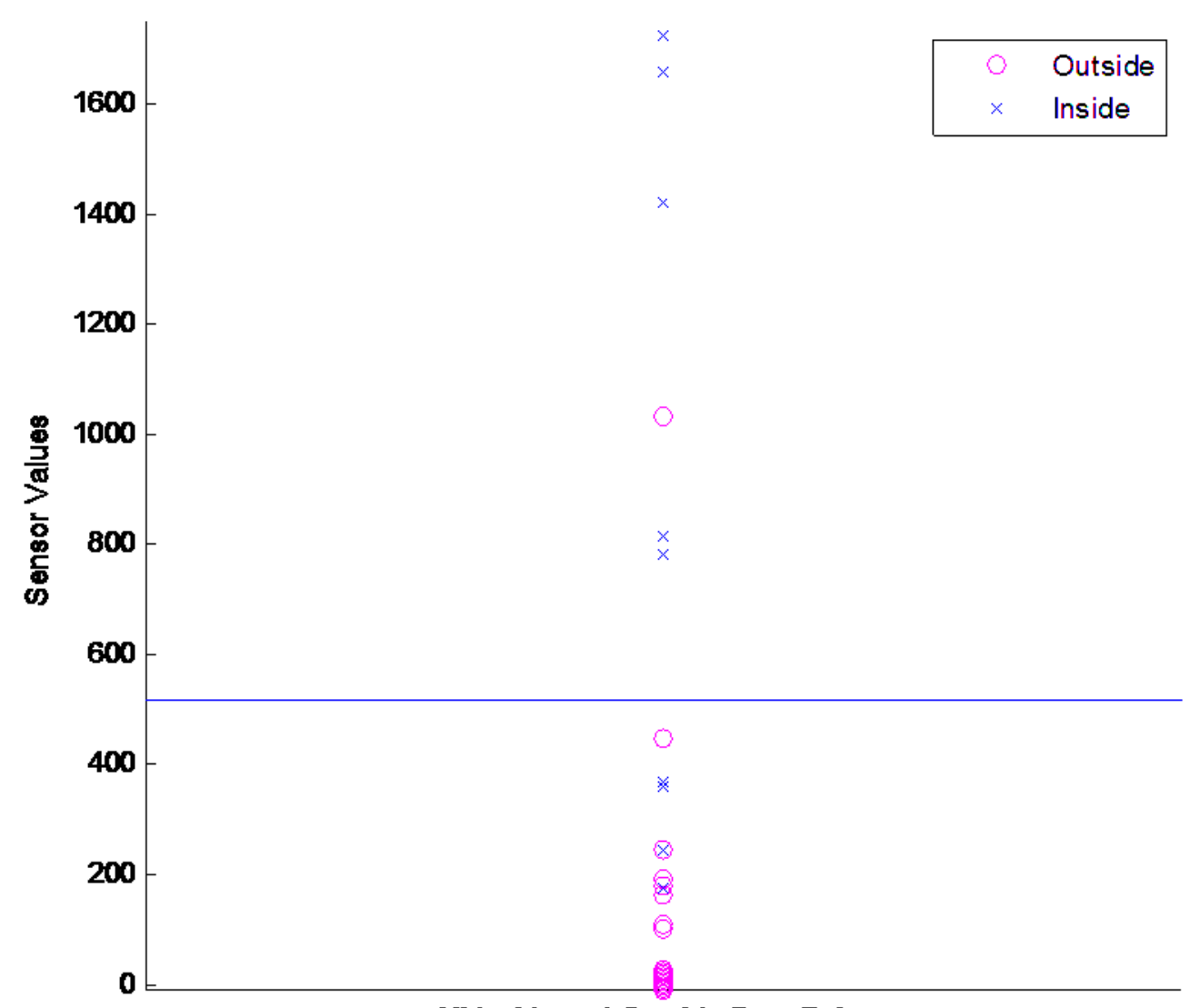

All Inside and Outside Data Pcints

Figure 32: Significant (Inside) verses insignificant (Outside) sensor scores as subjected to a classifier

The individualized thresholds were applied to pressure change data; sensors with a baseline value less than the thresholds were ignored. Within each grouping, instances during which at least two sensors were relieved of $95 \%$ of the original baseline pressure 
were identified. Instances were defined to be a time difference between relieved sensors of less than half a second. The algorithm then conditionally separated Scenario 1 enactments from Scenario 2 enactments; if no double incidences of pressure relief occurred, then the enactment was determined to be of Scenario 1. If double incidences of relief occurred, the scenario was determined to be Scenario 2.

\subsection{Algorithmic Results and Discussion}

The first stage of DBLS determined if the partipant enactment in question was of Scenario 3: lying-sitting. This was done by calculating the sum of sensor values in the top mat, determining a baseline pressure value, finding the pressure change from baseline at every point in time, and determining if that signal dropped permanently by $80 \%$. An example of a correctly identified Scenario 3 enactment is seen in Figure 33.

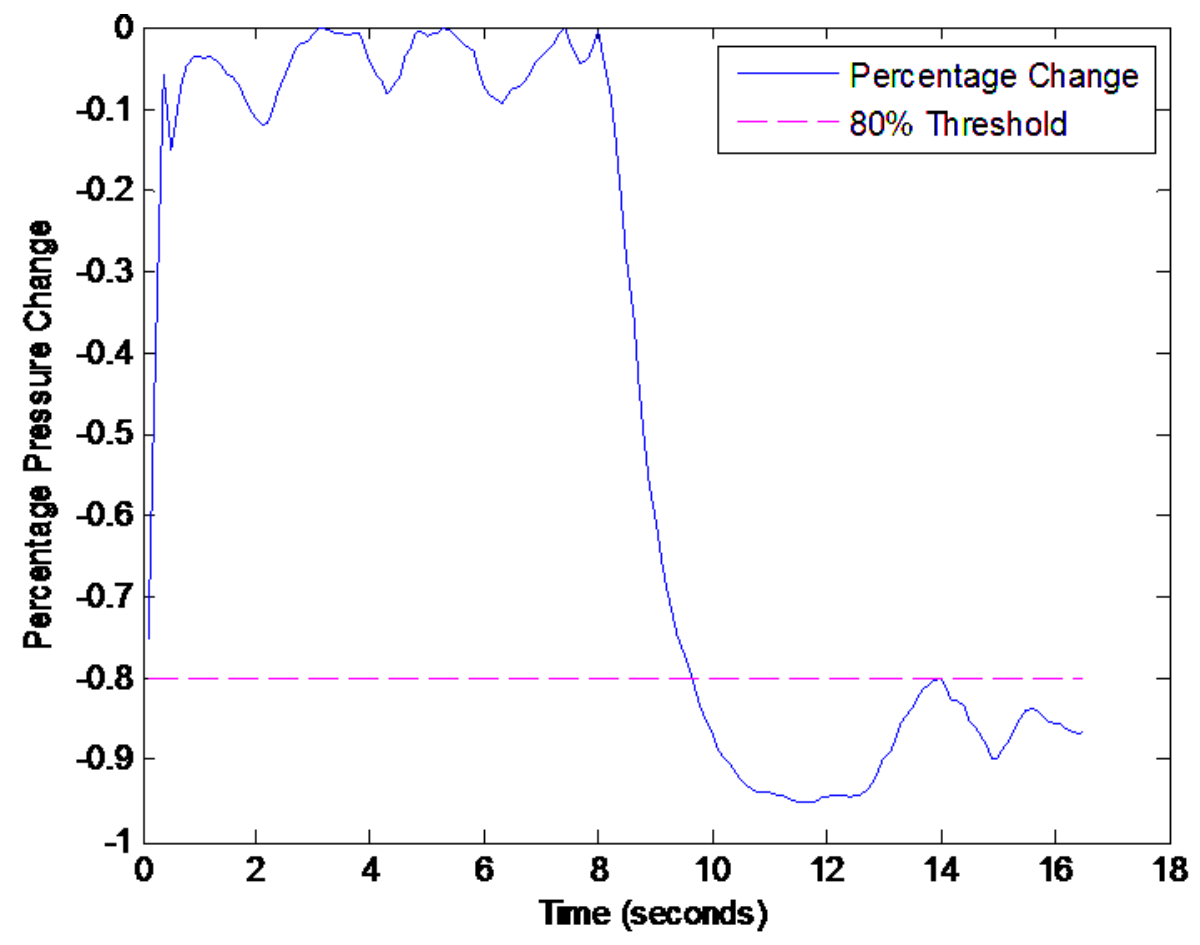

Figure 33: Percentage pressure change from baseline for the top mat. 
The secondary stage of DBLS determined if the enactment was of either Scenario 1 or Scenario 2. This was done by calculating percentage increase and percentage decrease, determining sensors of significant score outputs and identifying incidences during which at least two sensor outputs dropped by $95 \%$. While this calculation was done for all groupings described in Section 6.3, only the results from the middle mat (Mat B) were required to distinguish Scenario 1 from Scenario 2. An example of two simultaneous pressure reliefs from 5 sensors underneath the left hip, for one volunteer performing an enactment of Scenario 2 can be seen in Figure 34. The first incidence occurs at a time of 10 seconds, the second incidence occurs at a time of 22 seconds, and both incidences are highlighted with an arrow. The location of the relieved sensors (highlighted in yellow) on the left side of the middle mat can be seen in Figure 35.

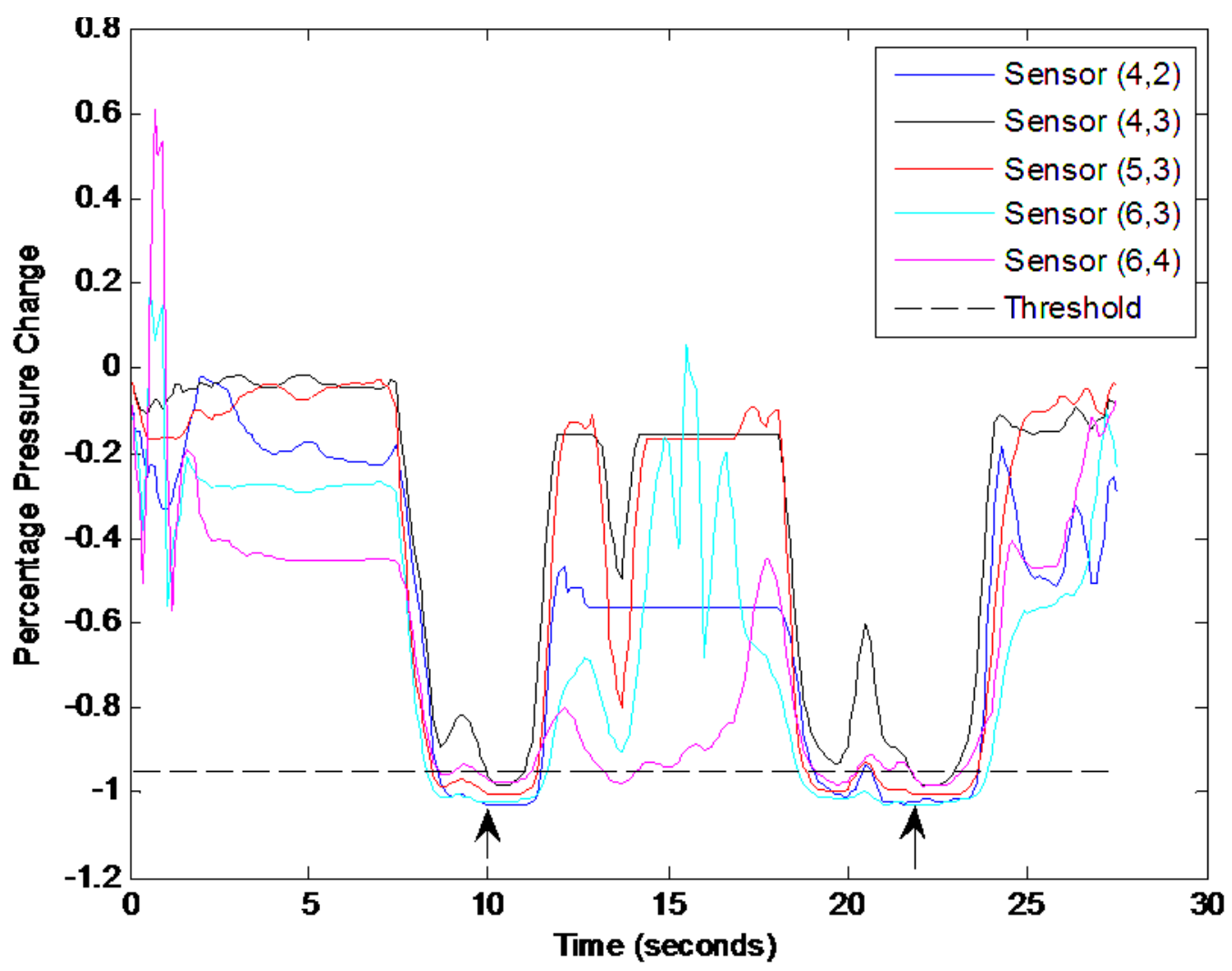

Figure 34: Two incidences of five simultaneous pressure sensors experiencing pressure relief. 


\begin{tabular}{|c|c|c|c|c|c|c|c|}
\hline $\begin{array}{l}\text { Left } \\
\text { Side }\end{array}$ & & & Head & of Bed & & & $\begin{array}{l}\text { Right } \\
\text { Side }\end{array}$ \\
\hline & & & & t A & & & \\
\hline$(1,1)$ & $(1,2)$ & $(1,3)$ & $(1,4)$ & $(1,5)$ & $(1,6)$ & $(1,7)$ & $(1,8)$ \\
\hline$(2,1)$ & $(2,2)$ & $(2,3)$ & $(2,4)$ & $(2,5)$ & $(2,6)$ & $(2,7)$ & $(2,8)$ \\
\hline$(3,1)$ & $(3,2)$ & $(3,3)$ & $(3,4)$ & $(3,5)$ & $(3,6)$ & $(3,7)$ & $(3,8)$ \\
\hline & & & & t B & & & \\
\hline$(4,1)$ & $(4,2)$ & $(4,3)$ & $(4,4)$ & $(4,5)$ & $(4,6)$ & $(4,7)$ & $(4,8)$ \\
\hline$(5,1)$ & $(5,2)$ & $(5,3)$ & $(5,4)$ & $(5,5)$ & $(5,6)$ & $(5,7)$ & $(5,8)$ \\
\hline$(6,1)$ & $(6,2)$ & $(6,3)$ & $(6,4)$ & $(6,5)$ & $(6,6)$ & $(6,7)$ & $(6,8)$ \\
\hline & & & & t $C$ & & & \\
\hline$(7,1)$ & $(7,2)$ & $(7,3)$ & $(7,4)$ & $(7,5)$ & $(7,6)$ & $(7,7)$ & $(7,8)$ \\
\hline$(8,1)$ & $(8,2)$ & $(8,3)$ & $(8,4)$ & $(8,5)$ & $(8,6)$ & $(8,7)$ & $(8,8)$ \\
\hline$(9,1)$ & $(9,2)$ & $(9,3)$ & $(9,4)$ & $(9,5)$ & $(9,6)$ & $(9,7)$ & $(9,8)$ \\
\hline
\end{tabular}

Figure 35: Schematic of the five sensors shown to have been relieved.

Overall results of DBLS can be observed in Table 9. The system assessed 81 scenarios as expected and 9 scenarios not as expected.

\begin{tabular}{|c|c|c|c|c|}
\hline & \multicolumn{3}{|c|}{ Real Category } \\
\hline & & Sc 1 & Sc 2 & Sc 3 \\
\hline \multirow{3}{*}{ 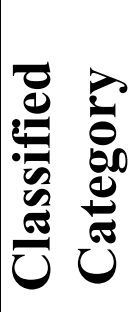 } & Sc 1 & 26 & 3 & 1 \\
\hline & Sc 2 & 4 & 27 & 1 \\
\hline & Sc 3 & 0 & 0 & 28 \\
\hline
\end{tabular}

Table 9: Classification results of DBLS 
Upon examination of video footage, it was found that the 4 data sets resulting from the enactment of Scenario 1, and misclassified as Scenario 2 were not assessed incorrectly. The participants performing these enactments had made mistakes in their movements and relieved pressure at the sacrum region though they had been instructed not to. The misclassification of Scenario 2 and Scenario 3, however, were errors made at this algorithmic stage of the system. The true accuracy of DBLS is therefore 85 correct classifications out of 90 , or $94 \%$ of the unidentified data sets, despite the ranging performances of scenarios by volunteers. 


\section{Chapter 7}

\section{Distinction Between Sitting Scenarios}

This chapter details a secondary stage of the algorithmic system; Distinction between Sitting Scenarios (DBSS).

\subsection{Introduction}

Distinction between Sitting Scenarios (DBSS) is depicted in Figure 14, and is discussed in the following sections. The first stage of the system, DIP aims to take raw, unidentified data and determine whether or not the subject's initial position is lying or sitting. If the initial position is determined to be sitting, all data generated by that participant and scenario is passed to this secondary stage. DBSS, depicted in Figure 36, aims to take data from DIP and determine which of two sitting to standing transfers the data represents; either Scenario 4: Independent but slow, or Scenario 5: Independent and vigorous.

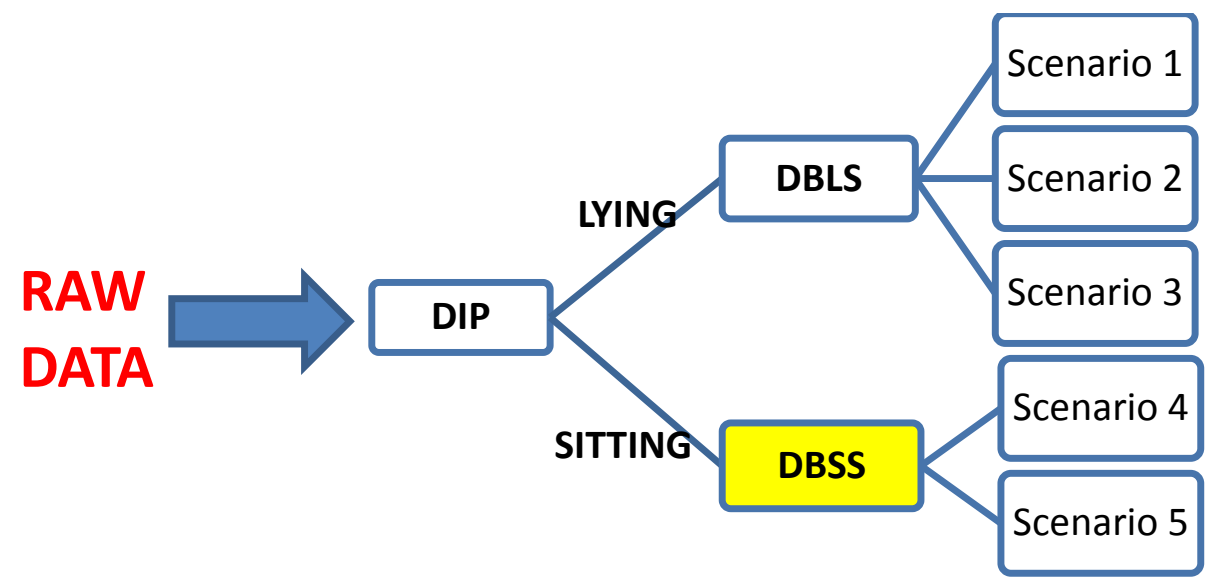

Figure 36: System tree describing the decision process for DBSS as it applies to the whole system. 
The sit-to-stand transfer has been examined in depth using many different methods. Specifically, using pressure mats and or force plates, the transfer has been broken down into phases to identify when the movement starts, ends, and how the movement was performed in order to characterize the transition [61]-[63]. In this thesis, the objective is to distinguish between a vigorous sit to stand transfer and a slower, less stable sit to stand transfer, not to characterize the general sit to stand transfer. A different approach is therefore taken in this chapter. The behaviour of the center of mass was examined for all patients and all scenarios. Features were extracted and evaluated by two different classifiers, the performance of the classifiers was evaluated, and a final model was determined for DBSS.

\subsection{Feature Extraction}

The first stage of this system, DIP, calculated a number of features from calibrated data generated by participants performing five different scenarios. These features included PAS, CLR, QLR, LonV, LatV, LonCP and LatCP as summarized in Table 5 and described in Chapter 5. If a set of data is identified as having an initial sitting position, two features, LonCP and LatCP, of the corresponding patient and scenario are passed to DBSS. These features describe the spatial movement pattern of the center of mass on the hospital mattress and pressure mats, an example of which can be seen in Figure 37 . The blue line describes the movement path of the center of mass for Participant 27, scenarios 4 and 5, and Figure 38 depicts the same image but zoomed, so the differences in movement paths are visible. 

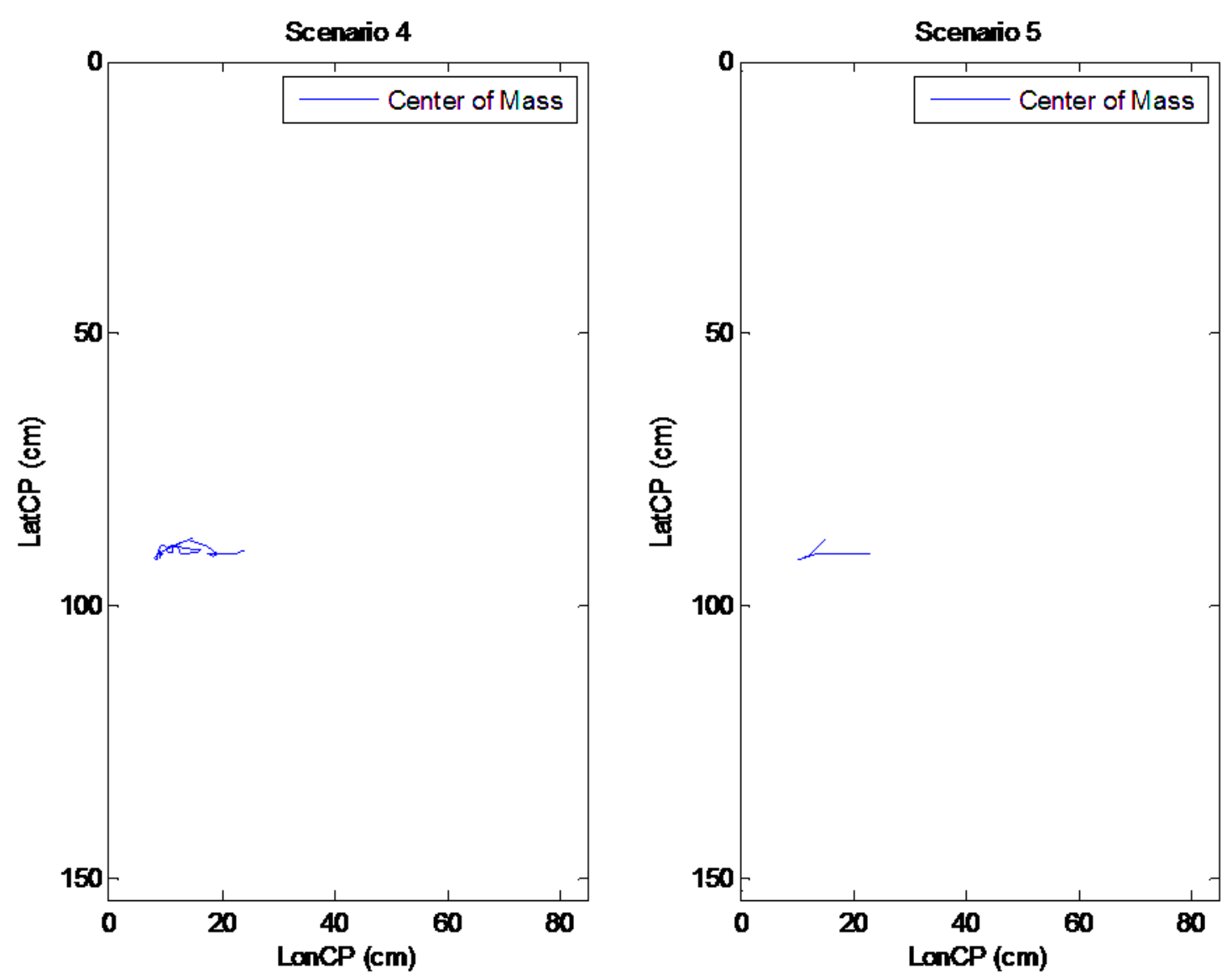

Figure 37: Mapped center of pressure for the duration of scenarios 4 and 5 .
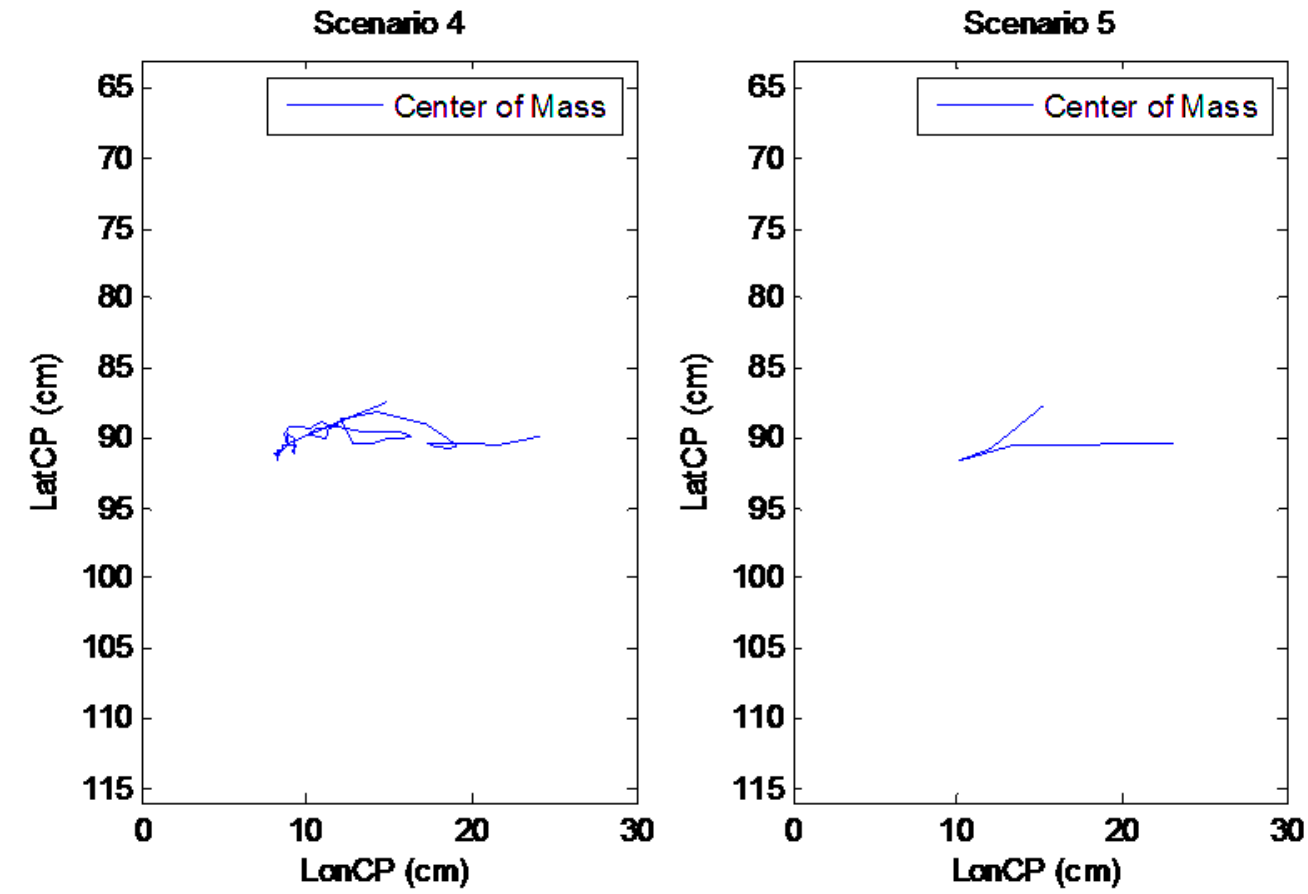

Figure 38: Zoomed, mapped center of pressure. 
From observation it can be seen that the center of mass movement path for Scenario 4 is more complex than the movement path for Scenario 5. Features examined in the following sections and summarized in Table 10 therefore aim to describe differences in center of mass path complexity. As opposed to Chapter 5, in which only the first 4 seconds of each scenario enacted by each participant is of interest, this chapter only considers data after the first 4 seconds of data, until the participant relieves all pressure from the hospital bed. Total pressure relief was identified as it was in Chapter 6; the point at which $95 \%$ of initial pressure had been relieved. $95 \%$ pressure relief was identified instead of total pressure to account for false positive pressure readings on the pressure mat from the hospital mattress shifting weight after pressure relief.

Table 10: Summary of features examined for DBSS

\begin{tabular}{|c|c|c|c|}
\hline Feature & $\begin{array}{c}\text { Feature } \\
\text { Acronym }\end{array}$ & $\begin{array}{c}\text { Chapter } \\
\text { Section }\end{array}$ & $\begin{array}{c}\text { Equation } \\
\text { Numbers }\end{array}$ \\
\hline \hline Sum of Vector Lengths & SVL & 7.2 .1 & $30-32$ \\
\hline Number of Acute Angles & NAA & 7.2 .2 & $33-35$ \\
\hline Start to Finish Vector & SFV & 7.2 .3 & 36 \\
\hline Longest Single Vector & LSV & 7.2 .4 & 37 \\
\hline Polygon Area & PA & 7.2 .5 & $38-39$ \\
\hline Number of Spatial Coordinates & NSC & 7.2 .6 & 40 \\
\hline
\end{tabular}

\subsubsection{Sum of Vector Lengths}

This feature aimed to enhance the differences in the movement paths for scenarios 4 and 5 by describing the distance travelled by the center of mass. The features LonCP and LatCP are equivalent to the $\mathrm{x}$ and $\mathrm{y}$ coordinates of the center of mass at every point in time for each participant and scenario. The movement trajectories depicted in Figure 37 and Figure 38 are created using these coordinates. The feature Sum of Vector Lengths (SVL) is the total sum of vector lengths between the center of mass coordinate points. For 
every point in time after 4 seconds and before the participant was identified to have relieved all pressure, there is a spatial coordinate point. In between each point is a vector, the Euclidean length for which was calculated as described in Equation 30, where $\boldsymbol{x}_{\boldsymbol{t}}$ and $\boldsymbol{y}_{\boldsymbol{t}}$ are the coordinates for the center of mass at time $\boldsymbol{t}, \boldsymbol{x}_{\boldsymbol{t}+\mathbf{1}}$ and $\boldsymbol{y}_{\boldsymbol{t}+\mathbf{1}}$ are the coordinates for the center of mass at time $\boldsymbol{t}+\mathbf{1}$, and the equation was calculated for each participant's data sets for scenarios 4 and 5. Significant vectors, considered to be any vector longer than 0.25 centimeters, were identified and summed for a total of 60 vector sums. These calculations are described in equations 31 and 32.

$$
\begin{gathered}
\text { length }\{p, s c\}(t)=\sqrt{\left(x_{t+1}\{p, s c\}-x_{t}\{p, s c\}\right)^{2}+\left(y_{t+1}\{p, s c\}-y_{t}\{p, s c\}\right)^{2}} \\
A(\operatorname{length}\{p, s c\}(t))=\left\{\begin{array}{lr}
\operatorname{length}\{p, s c\}(t) & \text { if length }\{p, s c\}(t)>0.25 \mathrm{~cm} \\
0 & \text { otherwise }
\end{array}\right. \\
S V L(p, s c)=\sum_{t=4}^{\text {end }} A(\operatorname{length}\{p, s c\}(t))
\end{gathered}
$$

\subsubsection{Number of Acute Angles}

This feature, Number of Acute Angles (NAA), aims to quantify the complexity of the center of mass movement path by determining the number of sharp directional changes in each movement path. As described in section 7.2.1, a spatial coordinate exists for all participants and scenarios at every point in time. Where the feature SVL calculated the length of every vector between pairs of consecutive coordinates, NAA calculates all angles between consecutive pairs of vectors. Angles were calculated by manipulating the dot product equation, a step function was applied allowing only angles less than $90^{\circ}$ to be considered, and the number of angles satisfying this condition was calculated as seen in equations 33,34 and 35 . 


$$
\begin{gathered}
\text { theta }\{p, s c\}(t)=\cos ^{-1} \frac{\text { length }\{p, s c\}(t) \cdot \text { length }\{p, s c\}(t+1)}{\| \text { length }\{p, s c\}(t)\|*\| \text { length }\{p, s c\}(t+1) \|} \\
A(\text { theta }\{p, s c\}(t))=\left\{\begin{array}{cc}
1 & \text { if theta }\{p, s c\}(t)<90^{\circ} \\
0 & \text { otherwise }
\end{array}\right. \\
N A A(p, s c)=\sum_{t=4}^{\text {end }} A(\operatorname{theta}\{p, s c\}(t))
\end{gathered}
$$

\subsubsection{Start to Finish Vector}

Participants were instructed to perform two separate sit to stand transfers. For the first sit to stand transfer, the participants were instructed to feign difficulty (Scenario 4) and in the second, the participants were instructed to stand as they normally would (Scenario 5). The scenario in which participants feigned difficulty ranged in performance, but generally involved an alternating use of hands to stand up. This feature therefore aimed to determine if bed exit location was different for scenarios 4 and 5 (relative to the start position) by calculating the Start to Finish Vector (SFV). This vector was calculated as it was in section 7.2.1, except the first and last coordinate points were used instead of many consecutive points, as seen in Equation 36.

$$
S F V(p, s c)=\sqrt{\left(x_{\text {last }}\{p, s c\}-x_{\text {first }}\{p, s c\}\right)^{2}+\left(y_{\text {last }}\{p, s c\}-y_{\text {first }}\{p, s c\}\right)^{2}}
$$

\subsubsection{Longest Single Vector}

The differences in scenario performances were designed so that the Scenario 5 transfer was completed faster than in Scenario 4. This feature aims to determine if the rate of change between points was also faster, or equivalently, longer. The single longest vector calculated in section 7.2.1 was identified as in Equation 37. 


$$
\operatorname{LSV}(p, s c)=\max (\text { length }\{p, s c\}(t))
$$

\subsubsection{Polygon Area}

This feature aimed to describe the differences in complexity of the center of mass movement paths by measuring the area of the polygons created during movements. This was calculated for each participant, scenarios 4 and 5, using polygon area equations where $\boldsymbol{a}$ is a variable indicating whether the area is intersecting (deducts from total area) or non-intersecting (contributes to total area). These calculations can be seen in equations 38 and 39.

$$
\begin{gathered}
P A(p, s c)=\frac{1}{2} \sum_{t=4}^{e n d} a *\left|\begin{array}{ll}
x_{t}\{p, s c\} & x_{t+1}\{p, s c\} \\
y_{t}\{p, s c\} & y_{t+1}\{p, s c\}
\end{array}\right| \\
a \begin{cases}-1, & \text { if area is intersecting } \\
1, & \text { if area does not intersect }\end{cases}
\end{gathered}
$$

\subsubsection{Number of Spatial Coordinates}

This feature, Number of Spatial Coordinates (NSC) aims to enhance the difference between the two sit to stand transfers of scenarios 4 and 5 by calculating the number of coordinate points that construct each movement path. This calculation can be seen in Equation 40, in which the size of the vector length array is found and increased by one because there is one more coordinate point than vectors. This was calculated for all participants and scenarios 4 and 5.

$$
N S C(p, s c)=\operatorname{size}(\text { length }\{p, s c\})+1
$$




\subsection{Feature Evaluation and Selection}

Feature evaluation was completed in this chapter as it was in Chapter 5; each feature was evaluated individually for its ability to distinguish between scenarios 4 and 5 . As in previous chapters, two classifiers were used to evaluate individual features in order to compare a simple verses a complex classifier in addition to evaluating feature performance. The classifiers used were an SVM classifier with a linear kernel and a linear discriminant classifier, both with leave-one-subject-out cross validation. After each feature was examined separately, the best performing features were further examined by adding Gaussian noise and re-evaluating feature performance. A final model was determined by using the best performing features with the best performing classifier and all of the patient and scenario data for scenarios 4 and 5 .

\subsection{Results and Discussion}

All features were evaluated individually using both a linear discriminant classifier and an SVM classifier with a linear kernel. The data from all participant's data sets from scenarios 4 and 5 were used to train each classifier iteratively. At each step data generated by all participants, with the exception of one, was used to train the classifiers and the trained classifier was then tested using data that was excluded from training. This iterative process included 30 steps, so as to test data from all subjects and train with the remaining data. The accuracy of each train and test iteration was recorded, averaged and can be seen in Table 6 . 
Table 11: Features examined and respective classifier accuracies.

\begin{tabular}{|c|c|c|}
\hline Feature & SVM Accuracy & Linear Accuracy \\
\hline \hline SVL & 0.85 & 0.80 \\
\hline NAA & 0.97 & 0.93 \\
\hline SFV & 0.55 & 0.57 \\
\hline LSV & 0.58 & 0.57 \\
\hline PA & 0.67 & 0.65 \\
\hline NSC & 0.98 & 0.93 \\
\hline
\end{tabular}

As with Chapter 5, when comparing the results between the simpler (linear) and more complex (SVM) classifier accuracies for individual features, they are very similar. Unlike Chapter 5, none of the features achieved total accuracy. Four of the features did not perform well enough to be considered: SVL, SFV, LSV and PA. The two remaining features, NAA and NSC were further examined using both classifiers to determine the effects of added Gaussian noise and feature combination on accuracy. Gaussian noise at an SNR rate of 10 and 5 was added to signals NAA and NSC to test the sensitivity of these features, then evaluated using both classifiers as seen in Table 12. The features were combined and evaluated by both classifiers as seen in Table 13.

Table 12: Accuracies of features evaluated by both classifiers.

\begin{tabular}{|c|c|c|c|c|}
\cline { 2 - 5 } \multicolumn{1}{c|}{} & \multicolumn{2}{c|}{ SVM Classifier Accuracy } & \multicolumn{2}{c|}{ Linear Classifier Accuracy } \\
\hline \hline Feature & SNR=10 & SNR=5 & SNR=10 & SNR=5 \\
\hline \hline NAA & 0.68 & 0.65 & 0.78 & 0.67 \\
\hline NSC & 0.80 & 0.70 & 0.80 & 0.68 \\
\hline
\end{tabular}

Table 13: Accuracies of combined features evaluated by both classifiers.

\begin{tabular}{|c|c|c|}
\hline \hline Combined Features & SVM Classifier Accuracy & Linear Classifier Accuracy \\
\hline \hline NAA \& NSC & 0.98 & 0.95 \\
\hline
\end{tabular}

The differences in accuracy between classifiers with noise added are again very similar, with the linear classifier generally having slightly more accurate results. The 
effect of combining the two features was the improvement of accuracy for both classifiers, with a difference of only 3 percent between the complex and simple models. The SVM classifier yielded the best results and so is the final model chosen for this stage of the system, depicted in Figure 39 and described in Equation 41. If this was a much larger data set, the percentage difference in accuracy may matter less than computational complexity and the linear discriminant classifier might be advantageous choice under those circumstances [64].

$$
y=-1.84 x+41.15
$$

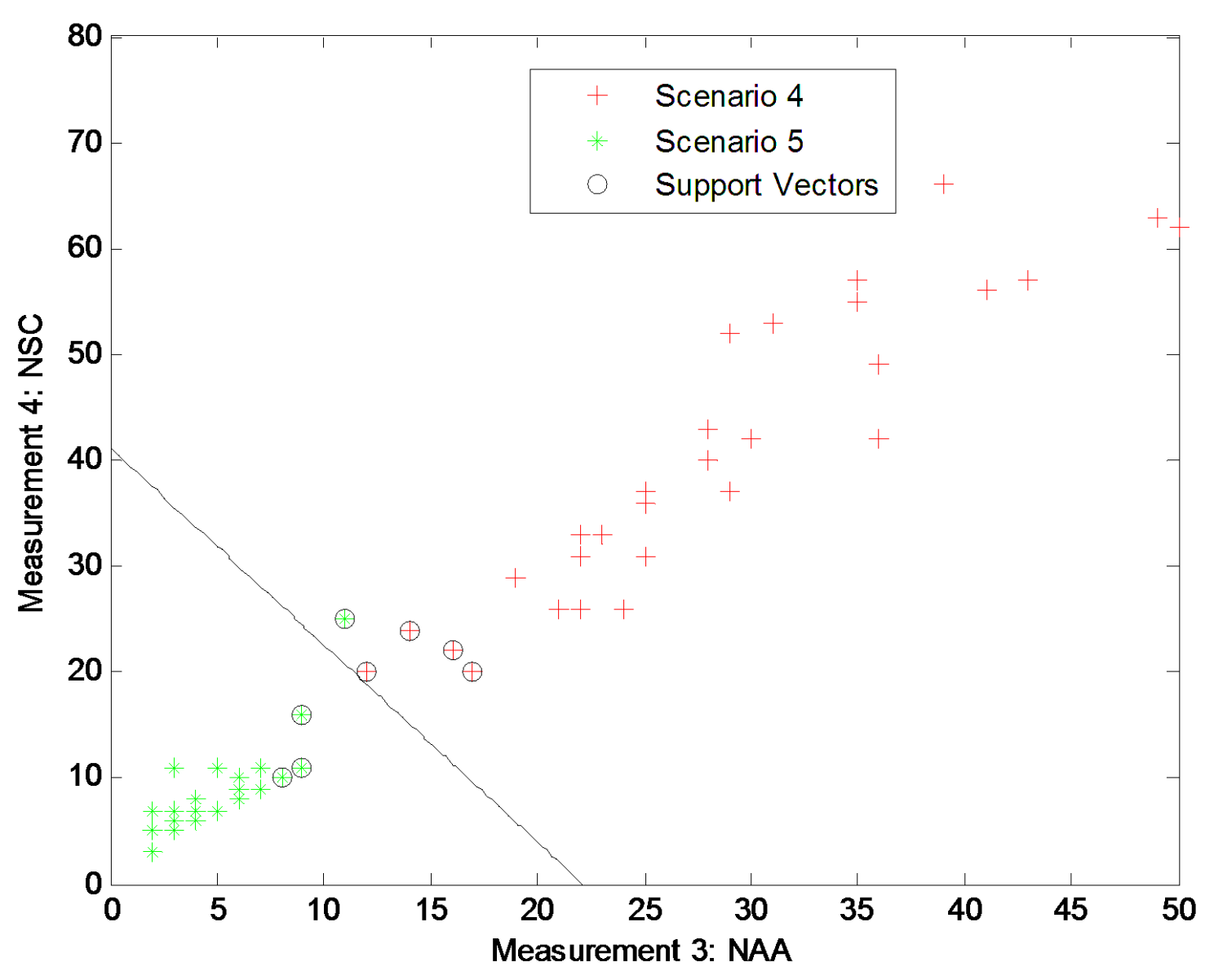

Figure 39: Final SVM model and resulting classifications 
The scenarios examined in this chapter were not as easily distinguishable as the orientations examined in Chapter 5. Upon review of the video footage, it was seen that many of the volunteers did not properly enact Scenario 4. Each participant was asked to perform a sit to stand transfer as an elderly person with lesser mobility and strength would. They were shown a demonstration video for guidance in which there was a lot of shifting weight between the hips and hands while shuffling forward on the bed. Some participants, instead of mimicking the video, performed a sit to stand transfer almost exactly as they normally would. This caused problems when trying to identify features that would distinguish the two scenarios, and resulted in slightly compromised classifier accuracies. 


\section{Chapter 8}

\section{System Performance and Conclusions}

This chapter discusses the overall performance of the system as described in Chapter 4, then discusses conclusions drawn from this thesis.

\subsection{System Performance}

Chapter 4 described the set-up of the algorithmic system aiming to take raw, unidentified data and determine which of five HABAM scenarios the data represents. The system was broken into three stages: a primary stage, Determination of Initial Posture (DIP), and two secondary stages, Distinction Between Lying Scenarios (DBLS) and Distinction Between Sitting Scenarios (DBSS). The results of individual stages are discussed in Chapters 4, 5 and 6, respectively. The overall results of this system can be seen in Table 14 in the form of true classifications verses classifications determined by the designed algorithmic system. 


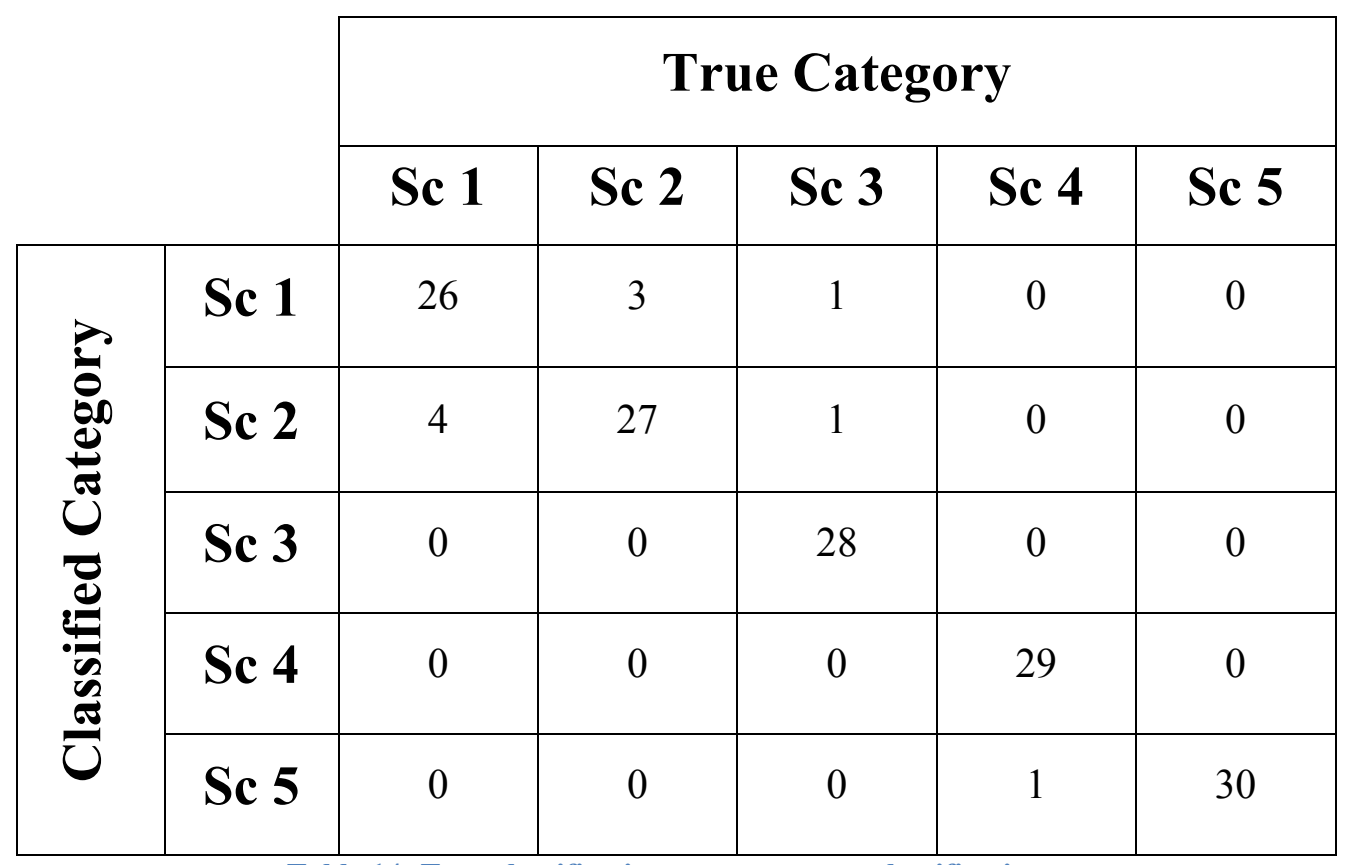

Table 14: True classifications verses system classifications.

The enactments of Scenario 1 misclassified as Scenario 2 were determined to be correctly identified pressure point relief. These enactments were therefore not considered to be misclassified when calculating the overall accuracy of the system. Of 150 enactments, 144 were classified correctly, yielding 96\% accuracy of the entire system. The majority of the misclassifications were due to inconsistencies in performance by participants.

\subsection{Thesis Conclusions}

The main objective of this thesis was to develop a system that automatically quantified the mobility of a patient in bed using pressure sensors to measure movement and the HABAM tool to quantify mobility. Thirty volunteers performed five enactments each on a standard hospital bed while pressure data was gathered from pressure mats underneath a hospital mattress. A system of algorithms was developed to process movement data and 
output the corresponding HABAM score. This system consisted of three main stages; a first stage, DIP and two secondary stages, DBLS and DBSS.

The first stage of the system, DIP, used both SVM and linear discriminant classifiers to determine ideal features and classification methods for identifying the initial position of a participant; a supine lying position, or a sitting position at the left center edge of the hospital bed. The ideal features were determined to be Latitudinal Variance (LatV) and the Percentage of Active Sensors (PAS), and the linear discriminant classifier was determined to be the optimal classification method. This optimal combination resulted in total accuracy.

Both secondary stages, DBLS and DBSS, received data from DIP and determined which specific scenario the data represented. If the data was determined to have an initial supine lying position, it was passed to DBLS and identified as one of scenarios 1, 2 or 3 . Scenario 1 is described as attempting, but unsuccessfully relieving pressure points from underneath a supine lying patient. Scenario 2 is described as attempting and successfully relieving pressure points, and Scenario 3 is described as transferring from a lying to sitting position. DBLS used percentage pressure change over time in different regions of the bed to identify these scenarios, resulting in $94 \%$ accuracy.

If the data was determined to have an initial sitting position, it was passed to the secondary stage, DBSS, and identified as one of scenarios 4 or 5 . Scenario 4 is described as transferring from a sitting position to a standing position slowly, and Scenario 5 is described as transferring from a sitting position to a standing position vigorously. DBSS used Longitudinal Center of Pressure (LonCP) and Latitudinal Center of Pressure (LatCP) to map the center of pressure over time. Features were extracted from this new 
signal, the best of which were the Number of Acute Angles (NAA) and the Number of Coordinate Points (NCP). An SVM classifier was then used for a final model with an accuracy of $98 \%$.

The overall system yielded $96 \%$ accuracy, where the misclassifications were due largely to inconsistencies in volunteer performance. In the first stage, there were little inconsistencies as the analyzed data was restricted to the time during which participants were sitting or lying quietly. The inconsistencies present at this stage were variations in orientation. These variations were accounted for in the final choice of variables; the two that were least dependant on patient orientation. The second stages aimed to determine specific scenarios. There was significantly more variation in participant performance at these stages. While participants were given the same instructions and shown a demonstrative video, all scenario enactments ranged in performance. Scenario 1 and 2 enactments ranged in direction, frequency and degree to which participants rolled. Scenario 3 was also enacted in a variety of different ways: some participants brought their bottom half towards their hands, others transferred to a seated position without the use of hands, some participants used their arms to sit only halfway up and others rolled to one side before pushing their upper bodies up with their hands. Scenario 4 enactments were often too similar to Scenario 5 enactments, and again performance ranged in the use of hands. While these variations were unexpected, difficult to overcome and resulted in a $4 \%$ error in total system performance, it was a challenge representative of unforeseen complications in future work with patients.

One interesting result found in this research, were 4 of the 10 enactments not assessed as expected, or misclassified. These particular enactments were of Scenario 1, 
but 'misclassified' as Scenario 2. Examination of video and pressure data revealed that the system had not assessed incorrectly, rather, the mats had captured an instance of pressure relief that went unnoticed. This result emphasizes the significance of ubiquitous computing in applications such as the HABAM, as data immeasurable to the eye is captured and recorded. Furthermore, this system, with relative engineering simplicity, was in some instances able to better assess HABAM scores than an observing researcher. This supports the idea that automation of the HABAM tool could introduce finer, clinically meaningful measures of immobility.

\subsection{Thesis Contributions}

In addition to the final conclusions, this thesis has provided four main contributions. The first contribution being preliminary work on frailty in elderly patients [21], and the collection of 150 sets of pressure data as well as 150 sets of corresponding video footage. The second contribution was the set of algorithms constituting the primary stage of the overall system, referred to as DIP. This stage used feature extraction and pattern classification to determine between one of two initial volunteer postures; a supine lying position and a sitting position [22],[23]. The third contribution was the set of algorithms constituting the first of two secondary stages of the overall system, referred to as DBLS. This stage used regions of interest and ratio calculations to identify incidences of pressure point relief and determine which of three scenarios (with an initial lying position) a given set of data represented [24],[25]. The fourth contribution was the set of algorithms constituting the second of the two secondary stages, referred to as DBSS. This stage calculated the center of pressure underneath subject, then used feature extraction and 
pattern classification to determine which of two scenarios (with an initial sitting position) a given set of data represented [26].

\subsection{Future Research}

The pressure mats used are capable of very fine measures of pressure and this system was designed with the intention of expansion through continued research, so within each of the stages described are areas of further examination. For example, DBLS was designed to detect whether a participant had relieved any pressure points, but is capable of determining which pressure point has been relieved, at what times and to what extent. The HABAM tool currently only assesses whether or not a patient can lift a pressure point, but the extent to which a patient can or cannot relieve these points can be explored and may be clinically meaningful. DBSS was designed to determine if a participant was standing vigorously or not, but with further analysis could be capable of incorporating assessments of balance in the sit to stand transfer, and possibly other transfers detailed in this thesis.

After further exploration of volunteer data, work will begin with patients. Data from pressure mats and additional sensors will be generated and collected from patients in an in-patient ward with the help and supervision of a health professional. This data will undergo extensive analysis with the goals of fully automating the HABAM tool and expanding the tool to include clinically meaningful but visually unidentifiable movements.

With the management of mobility progressions and declines in older adults often being overlooked, the number of geriatric patients increasing in an aging population, and 
the successful implementation of pressure sensing devices in other mobility related applications, full automation of HABAM could not only ensure consistent, reliable assessments and expand upon our knowledge of movement in the immobile, but could also emphasize the importance of pervasive computing in the assessment and tracking of elderly mobility and immobility. 


\section{References}

[1] Baklinski, Thaddeus M. (2010) "Statistics Canada Predicts Seniors Will Soon Outnumber Children," Available: http://www.lifesitenews.com/ldn/2010/may/10052704.html

[2] Statistics Canada, "The canadian population in 2011: age and sex," Statistics Canada, Canada, Ottawa, 2011.

[3] Institute of Aging, "The future is aging: institute of aging strategic plan 20072012," Canadian Institute of Health Research (CIHR), Canada, Vancouver, 2007.

[4] Institute of Aging, "Living longer, living better: institute of aging strategic plan 2013-2018," Canadian Institute of Health Research (CIHR), Canada, Vancouver, 2013.

[5] P. Crome, and F. Lally, "Frailty: joining the giants," Can. Med. Assoc. J., vol. 183, no. 8, pp. 889-890, April 2011.

[6] S. K. Inouye, S. Studenski, M. E. Tinetti, and G. A. Kuchel, "Geriatric syndromes: clinical, research, and policy implications of a core geriatric concept," J. Am. Geriatrics Soc., vol. 55, no. 5, pp. 780-791, May 2007.

[7] D. Davis, M. Rockwood, A. Mitnitski, K. Rockwood, "Impairments in mobility and balance in relation to frailty," Arch. Geront. Geriatr., vol. 53, no. 1, pp. 79-83.

[8] K. Rockwood, and A. Mitnitski, "Frailty defined by deficit accumulation and geriatric medicine defined by frailty," Clin. Geriatr. Med., vol. 27, no. 1, pp. 17-26, Feb. 2011. 
[9] K. Rockwood, M. Rockwood, M. Andrew, and A. Mitnitski, "Reliability of the hierarchical assessment of balance and mobility in frail older adults," J. Am. Geriatrics Soc., vol. 56, no. 7, pp. 1213-1217, July 2008.

[10] R. Hubbard, and K. Rockwood, "Mobility and balance in older people," Can. J. Gen. Intern. Med., vol. 5, no. 1 pp. 13-15, April 2010.

[11] R. Hubbard, E. Eeles, M. Rockwood, N. Fallah, E. Ross, A. Mitnitski, and K. Rockwood, "Assessing balance and mobility to track illness and recovery in older inpatients," J. Gen. Intern. Med., vol. 26, no. 12, pp. 1471-1478, Dec. 2011.

[12] E. Martini, N. Garrett, T. Lindquist, and G. Isham, "The boomers are coming: a total cost of care model of the impact of population aging on health care costs in the united states by major practice category," Health Services Research, vol. 42, no. 1, pp. 201-218, Feb. 2007.

[13] L. Walsh, S. McLoone, J. Behan, and T. Dishongh, "The deployment of a nonintrusive alternative to sleep/wake wrist actigraphy in a home-based study of the elderly," Conf. Proc. IEEE Eng. Med. Biol. Soc., pp. 1687-1690, 2008.

[14] A. Adami, M. Pavel, T. Hayes, A. Adami, and C. Singer, "A method for classification of movements in bed," Conf. Proc. IEEE Eng. Med. Biol. Soc., pp. 7881-7884, 2011

[15] M. Marschollek, M. Goevercin, K. Wolf, B. Song, M. Gietzelt, R. Haux, and E. Steinhagen-Thiessen, “A performance comparison of accelerometry-based step detection algorithms on a large, non-laboratory sample of healthy and mobilityimpaired persons," Conf. Proc. IEEE Eng. Med. Biol. Soc., pp. 1319-1322, 2008. 
[16] R. Okuno, S. Fujimoto, J. Akazawa, M. Yokoe, S. Sakoda, and K. Akazawa, "Analysis of spatial temporal plantar pressure pattern during gait in Parkinson's disease," Conf. Proc. IEEE Eng. Med. Biol. Soc., pp. 1765-1768, 2008.

[17] C. Gehin, E. Brusseau, R. Meffre, P. Schmitt, J.F. Deprez, and A. Dittmar, "Which techniques to improve the early detection and prevention of pressure ulcers?," Conf. Proc. IEEE Eng. Med. Biol. Soc., pp. 6057-6060, 2006.

[18] R. Meffre, C. Gehin, and A. Dittmar, "MAPI: Active interface pressure sensor integrated into a seat," Conf. Proc. IEEE Eng. Med. Biol. Soc., pp. 1358-1361, 2007.

[19] H. Jang, J. Jang, and S-J. Park, "Daily activity recognition system for the elderly using pressure sensors," Conf. Proc. IEEE Eng. Med. Biol. Soc., pp. 5188-5191, 2008.

[20] M. Alwan, G. Demiris, Z. He, J. Keller, M. Skubic, and M. Rantz, “Technology for successful aging," Conf. Proc. IEEE Eng. Med. Biol. Soc., pp. 3290-3293, 2006.

[21] S. Bennett, X. Song, A. Mitnitski, K. Rockwood, "A Limit to Frailty in Very Old, Community-Dwelling People: A Secondary Analysis of the Chinese Longitudinal Health and Longevity Study," Age and Ageing, pp. 1-6, December 2012.

[22] S. Bennett, R. Goubran, K. Rockwood, A. Arcelus, and F Knoefel, "Pressure Signal Feature Extraction for the Differentiation of Clinical Mobility Assessments," in Proc. IEEE Med. Meas. Appl. (MeMeA), pp. 1-5, May 2012.

[23] S. Bennett, R. Goubran, K. Rockwood, and F Knoefel, "Automated Assessment of Mobility in Bedridden Patients," in Proc. IEEE Eng. Med. Biol. Soc. (EMBS), pp. 4271-4274, July 2012. 
[24] S. Bennett, R. Goubran, K. Rockwood, and F Knoefel, "Monitoring the Relief of Pressure Points for Pressure Ulcer Prevention: A Subject Dependant Approach,” in Proc. IEEE Med. Meas. Appl. (MeMeA), pp. 135-138, May 2013.

[25] S. Bennett, R. Goubran, K. Rockwood, and F Knoefel, "Considering Center of Gravity, in Pressure Feature Extraction From Mobility Assessments," presented at the 2nd annual conference of Healthcare Support through Information Technology Enhancements (hSITE), Montreal, Quebec, 2012.

[26] S. Bennett, R. Goubran, K. Rockwood, and F Knoefel, "Distinguishing Between Stable and Unstable Sit-To-Stand Transfers Using Pressure Sensors," submitted to IEEE Int. Symp. on Medical Measurements and Applicat. (MeMeA).

[27] A. Malin, "Review series: old age: pneumonia in old age," Chronic Respiratory Dis., vol. 8, no. 3, pp. 207-210, Aug. 2011.

[28] A. H. Snijders, W. B. P. Van, N. Giladi, and B. R. Bloem, "Neurological gait disorders in elderly people: clinical approach and classification." Lancet Neurol., vol. 6, no. 1 pp.63-74, 2007.

[29] B. R. Bloem, J. Haan, A. M. Lagaay, W. Beek, A. R. Wintzen, and R. A. Roos, "Investigation of gait in elderly subjects over 88 years of yge." J. Geriatric Psych. and Neurol. vol. 5, no. 2, 1992.

[30] F. Onen, M. C. Henry-Feugeas, C. Roy, G. Baron and P. Ravaud. "Mobility decline of unknown origin in mild cognitive impairment: an MRI-based clinical study of the pathogenesis." Brain Research, vol. 12, no. 22, pp. 79, 2008.

[31] K. Rockwood and A. Mitnitski, "Frailty, fitness, and the mathematics of deficit accumulation." Reviews in Clin. Gerontology, vol. 17, no. 1, pp. 1-12, 2007. 
[32] K. G. Abellan, Y. Rolland, H. Bergman, J. E. Morley, S. B. Kritchevsky and B. Vellas, "The I.A.N.A task force on frailty assessment of older people in clinical practice," J. Nutrition, Health \& Aging, vol. 12, no. 1, pp. 29-37, 2008.

[33] J. Walston, E. C. Hadley, L. Ferrucci, J. M. Guralnik, A. B. Newman, S. A. Studenski, W. B. Ershler, T. Harris and L. P. Fried, "Research agenda for frailty in older adults: toward a better understanding of physiology and etiology: summary from the american geriatrics society/national institute on aging research conference on frailty in older adults." J. Am. Geriatrics Soc., vol. 54, no. 6,pp. 991-1001, 2006.

[34] L. P. Fried, C. M. Tangen, J. Walston, A. B. Newman, C. Hirsch, J. Gottdiener, T. Seeman, R. Tracy, W. J. Kop and G. Burke, "Frailty in older adults: evidence for a phenotype." J. Gerontol. Series A., vol 56, 2001.

[35] A. B. Mitnitski, A. J. Mogilner and K. Rockwood. "Accumulation of deficits as a proxy measure of aging." The Scientific World Journal, vol. 1, pp. 323-336, 2001.

[36] N. F. Woods, A. Z. LaCroix, S. L. Gray, A. Aragaki, B. B. Cochrane, R. L. Brunner, K. Masaki, A. Murray and A. B. Newman. "Frailty: emergence and consequences in women aged 65 and older in the women's health initiative observational study." J. Am.Geriatrics Soc., vol. 53, no. 8, pp. 1321-1330, 2005.

[37] K. J. Ottenbacher, G. V. Ostir, M. K. Peek, S. A. Snih, M. A. Raji and K. S. Markides. "Frailty in older mexican americans." J. Am.Geriatrics Soc., vol. 53, no. 9, pp. 1524-1531, 2005.

[38] D. Rowell, D. N. Wormley and R. K. Yedavalli, "System dynamics: an introduction." Applied Mechanics Reviews, vol. 50, no. 6, 1997. 
[39] C. MacKnight and K. Rockwood. "Rasch analysis of the hierarchical assessment of balance and mobility (HABAM)." J. Clin. Epidemiology., vol. 53, no. 12, pp. 1242$1247,2000$.

[40] C. MacKnight, A. Sibley and K. Rockwood. "The sensibility of bedside tests of balance and mobility." Geriatrics Today., vol. 5, no. 3, pp. 140-143, 2002.

[41] D. Podsiadlo, S. Richardson, "The timed "up \& go": a test of basic functional mobility for frail elderly persons," J. Am. Geriatrics Soc., vol. 39, no. 2, pp. 142148, Feb. 1991.

[42] M. E. Tinetti, "Performance-oriented assessment of mobility problems in elderly patients," J. Am. Geriatrics Soc., no. 34, pp. 119-126, 1986.

[43] K. O. Berg, S. Wood-Dauphinee, J. I. Williams, D. G. Gayton, "Measuring balance in the elderly: preliminary development of an instrument," Physiother. Can., no. 41, pp. 304-311, 1989.

[44] P. W. Duncan, D. K. Weiner, J. Chandler, and S. Studenski, "Functional reach: anew clinical measure of balance," J. Gerontol: Med. Sc., vol. 45, no. 6, pp.192197, 1990.

[45] C. Orwat, A. Graefe, and T. Faulwasser, "Towards pervasive computing in health care - a literature review," BMC Med. Inform. Decis. Mak., vol. 8, no. 26, June 2008.

[46] S. Sneha and U. Varshney U, "Enabling ubiquitous patient monitoring: model, decision protocols, opportunities and challenges". Decision Support Systems, vol. 46, no. 3 pp. 606-619, 2009. 
[47] S. Sneha and U. Varshney U, "Enabling ubiquitous patient monitoring: model, decision protocols, opportunities and challenges". Decision Support Systems, vol. 46, no. 3 pp. 606-619, 2009.

[48] V. H. Cheung, L. Gray and M. Karunanithi, "Review of accelerometry for determining daily activity among elderly patients," Archives of Physical Medicine and Rehabilitation, vol. 92, no. 6, pp. 998-1014, 2011.

[49] D. I. Townsend, R. Goubran, M. Frize, and F. Knoefel, "Preliminary results on the effect of sensor position on unobtrusive rollover detection for sleep monitoring in smart homes," in Proc. IEEE Eng. Med. Biol. Soc., pp. 6135-6138, Sept. 2009.

[50] M. H. Jones, R. Goubran and F. Knoefel, "Identifying movement onset times for a a bed-based pressure sensor array," in Proc. IEEE Med. Meas. Appl. (MeMeA), pp. 111-114, April 2006.

[51] M. H. Jones, R. Goubran and F. Knoefel, "Reliable Respiratory Rate Estimation from a Bed Pressure Array." Proc. IEEE Eng. Med. Biol. Soc. pp. 6410-6413, 2006.

[52] V. Mishra, N. Singh, U. Tiwari and P. Kapur, "Fiber Grating Sensors in Medicine: Current and Emerging Applications," Sensors \& Actuators: A. Physical, vol. 167, no. 2, pp. 279-290, 2011.

[53] A. G. Mignani and F Baldini, "Biomedical sensors using optical fibers," Rep. Prog. Phys., vol. 59, pp. 1-28, 1996.

[54] A. G. Mignani and F Baldini, "Biomedical sensors using optical fibers," Rep. Prog. Phys., vol. 59, pp. 1-28, 1996. 
[55] D. G. Caldwell and C. Gosney, "Multimodal tactile sensing and feedback (teletaction) for enhanced telemanipulator control," in Conf. Proc. IEEE/RSJ Intelligent Robots Systems, pp. 1487-1494, July 1993.

[56] Y. D. Jia and K. J. Li, "A high resolution and high compliance tactile sensing array", Chinese Science Bulletin, vol. 39, no. 15, pp. 1249-1252, 1994.

[57] J. H. Porter, "Differential fiber optic proximity sensor", US Patent 4,358,960, 1982.

[58] E. M. Reimer and L.H. Baldwin, "Cavity sensor technology for low cost automotive safety and control devices," in Conf. Air Bag Tech., 1999.

[59] N. Foubert, "Posture recognition and postural transition detection using bed-based pressure sensor arrays," M.A.Sc. thesis, Dept. of Computer and Systems Engineering, Carleton University, Ottawa, ON, 2010.

[60] A. Arcelus, "Context-aware smart home monitoring through the analysis of pressure sequences," PhD thesis, Dept. of Computer and Systems Engineering, Carleton University, Ottawa, ON, 2010.

[61] A. Arcelus, R. Goubran, F. Knoefel, H. Sveistrup, and M. Bilodeau, "Detection of bouncing during sit-to-stand transfers with sequential pressure images," in Proc. IEEE Med. Meas. Appl. (MeMeA), pp. 158-161, May 2011.

[62] A. Arcelus, I. Veledar, R. Goubran, F. Knoefel, M. Bilodeau, and H. Sveistrup, "Measurements of sit-to-stand timing and symmetry from bed pressure sensors,' IEEE Trans. Instrum. Meas., vol. 60, no. 5, pp. 1732-1740, May 2011.

[63] K. Sakai, G. Nakagami, N. Matsui, H. Sanada, A. Kitagawa, E. Tadaka, and J. Sugama, "Validation and determination of the sensing area as part of development 
of a new mattress with an interface pressure-sensing system," Biosci. Trends, vol. 2, no. 1, pp. 36-43, Feb 2008

[64] S. M. Weiss, C. S. Kulikowski, "How to estimate the true performance of a learning system," in Computer systems that learn: classification and prediction methods from statistics, neural nets, machine learning and expert systems, 1 st ed. San Francisco, CA, USA: Morgan Kaufmann Publishers, 1991, ch. 2, sec. 2.7-2.8, pp. 39-43. 
Appendix A 


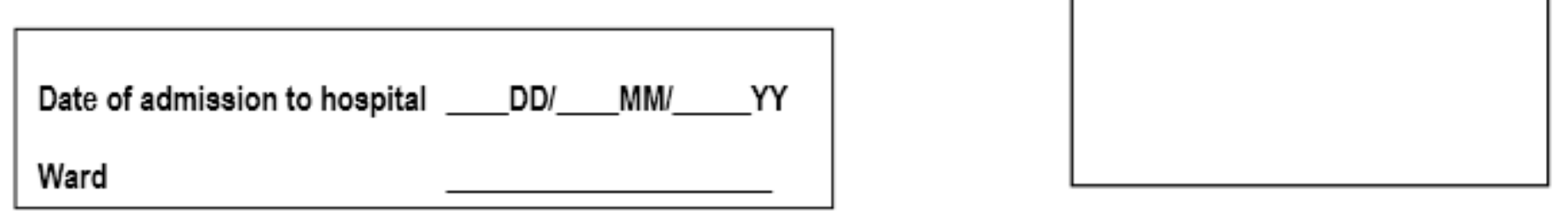

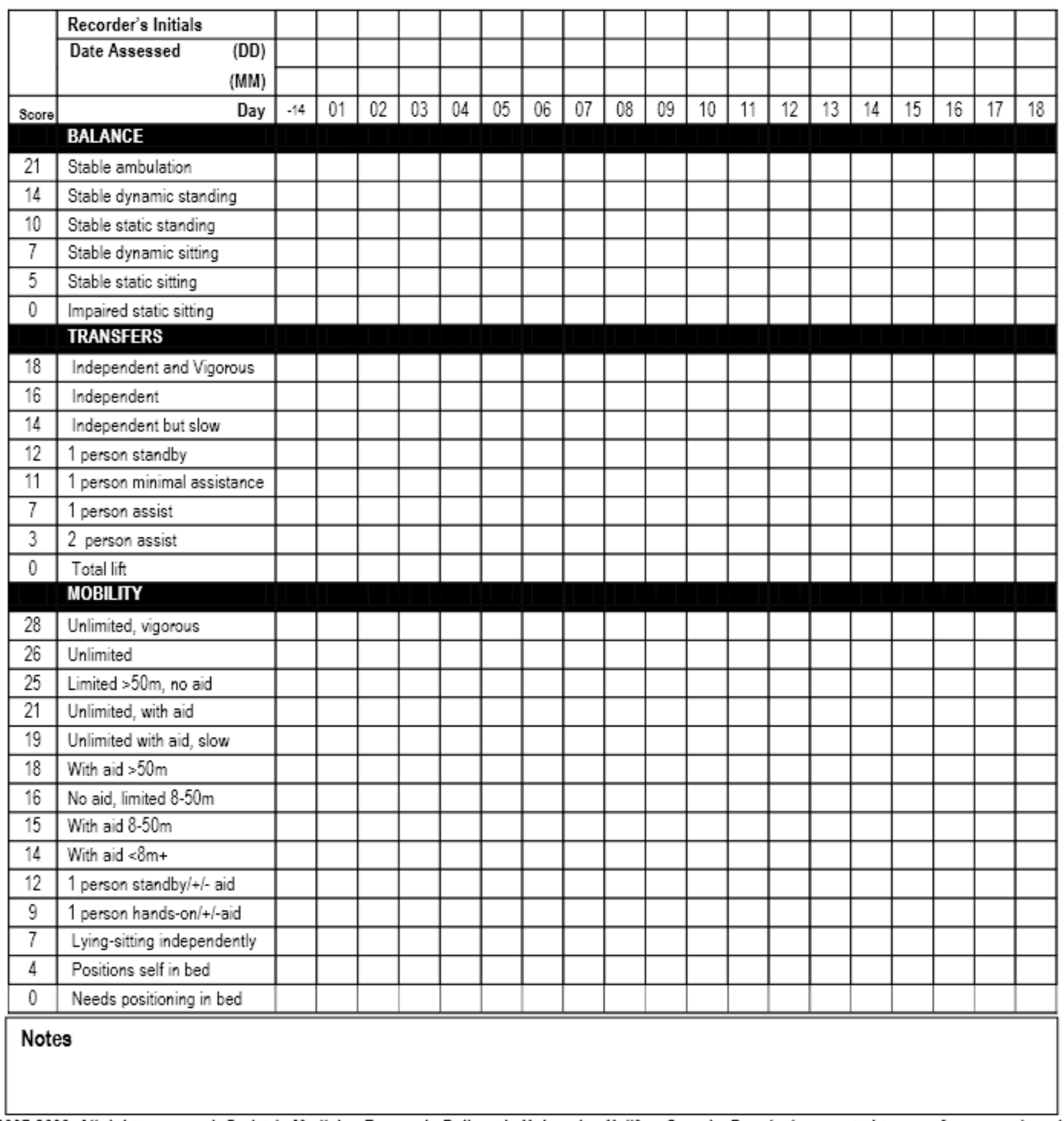

(9) 2007-2009. All rights reserved. Geriatric Medicine Research, Dalhousie University, Halifax, Canada. Permission granted to copy for research and educational use only. Version 1.1 